\title{
Elasticity of basic structural element in anisotropic macromolecular networks
}

\author{
Dissertation \\ zur Erlangung des \\ mathematisch-naturwissenschaftlichen Doktorgrades \\ "Doctor rerum naturalium" \\ der Georg-August-Universität Göttingen \\ im Promotionsprogramm ProPhys \\ der Georg-August University School of Science (GAUSS)
}

vorgelegt von

Mohammadhosein Razbin Khalilabad

aus Ardebil, Iran

Göttingen, 2016 


\section{Betreuungsausschuss}

Prof. Dr. Annette Zippelius,

Institut für Theoretische Physik,

Georg-August-Universität Göttingen

Prof. Dr. Reiner Kree,

Institut für Theoretische Physik,

Georg-August-Universität Göttingen

\section{Mitglieder der Prüfungskommission}

Referentin: Prof. Dr. Annette Zippelius, Institut für Theoretische Physik,

Georg-August-Universität Göttingen

Korreferent: Prof. Dr. Reiner Kree, Institut für Theoretische Physik,

Georg-August-Universität Göttingen

\section{Weitere Mitglieder der Prüfungskommission}

Prof. Dr. Marcus Müller,

Institut für Theoretische Physik,

Georg-August-Universität Göttingen

Prof. Dr. Sarah Köster,

Institut für Röntgenphysik,

Georg-August-Universität Göttingen

Priv.-Doz. Dr. Salvatore R. Manmana,

Institut für Theoretische Physik,

Georg-August-Universität Göttingen

Prof. Dr. Stefan Klumpp,

Institut für Nichtlineare Dynamik ,

Georg-August-Universität Göttingen

Tag der mündlichen Prüfung: 28.July.2016 
To my beloved Family... 



\section{Contents}

Page

1 Introduction 1

2 Wormlike chain model and weakly bending approximation 5

2.1 Wormlike chain model . . . . . . . . . . . . . . . 5

2.2 Weakly bending approximation . . . . . . . . . . . . . . 6

2.2.1 Weakly bending approximation for calculation of the orientationalpositional distribution function of semiflexible filament . . . . 7

$2.2 .2 \quad$ Weakly bending approximation in the problem of filament with a discontinuous tension . . . . . . . . . . . . . 7

3 Mechanical properties of branched actin filaments 9

3.1 The model: semiflexible branched filaments in the weakly bending limit 9

3.2 Properties of single branched filaments . . . . . . . . . . . . . 12

3.3 Extension to the F-actin network: Properties of an ensemble of branched filaments . . . . . . . . . . . . . . . . . . . . . . . . . . . .

3.4 Summary $\ldots \ldots \ldots \ldots \ldots \ldots$. . . . . . . . . . . . . . . . . . . 20

4 Mechanical properties of Filament with kinks 23

4.1 The probability of finding the end tip of the filament with one kink at fixed position . . . . . . . . . . . . . . 23

4.2 The probability density function of the regularly kinked filament . . . 25

4.3 The force-extension relation in the fixed extension ensemble . . . . . 26

4.4 The force exerted on a restricting wall . . . . . . . . . . . . . . . 27

4.5 The force associated with the filament with one kink . . . . . . . . . 27

4.6 The force exerted by the regularly kinked filament . . . . . . . . . . . . 28

4.7 Summary . . . . . . . . . . . . . . . . . . . . 31

5 Elasticity of a semiflexible filament with a discontinuous tension due to motor protein 33

5.1 Model description . . . . . . . . . . . . . . . . . . 33

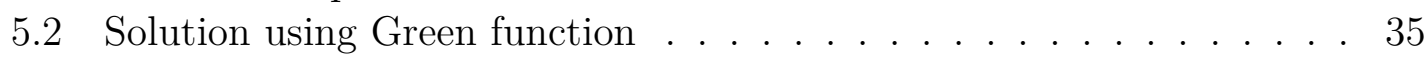

5.2 .1 Clamped-free filament . . . . . . . . . . . . . . . . 36

5.2 .2 Hinged-hinged filament . . . . . . . . . . . . . . . 36 
$5.3 \quad$ Analytical Results . . . . . . . . . . . . . . . . . . . . . . . 37

5.3 .1 Limit of small motor force $f_{m} \ldots \ldots$. . . . . . . . . . 39

5.3 .2 Limit of large motor force $f_{m} \ldots \ldots \ldots$. . . . . . . . 41

5.3 .3 Limit of large force $f_{\text {ext }}$ and $f_{m}=-f_{\text {ext }}+\epsilon$. . . . . . . . 43

5.4 Relation to single-motor experiments . . . . . . . . . . . . . . . . 45

5.5 Summary . . . . . . . . . . . . . . . . . . 46

$\begin{array}{lll}6 & \text { Conclusion and summary } & 47\end{array}$

\begin{tabular}{ll}
\hline Appendices & 49
\end{tabular}

A Mechanical properties of branched actin filaments 51

A.1 Tilted filament . . . . . . . . . . . . . . . . . . . . 51

A.2 Branched filament. . . . . . . . . . . . . . . . . . 51

A.3 Parameter values . . . . . . . . . . . . . . . . . . . . . . . 52

B Mechanical properties of Filament with kinks

B.1 Kinked filament with even number of arms . . . . . . . . . . . . . . . 55

B.2 Kinked filament with odd number of arms . . . . . . . . . . . . . . . 56

B.3 The general case . . . . . . . . . . . . . . . . . 56

C The equivalence of the rigid quantum rotor and semiflexible filament $\quad 59$

D Elasticity of a semiflexible filament with tension discontinuity $\quad \mathbf{6 1}$

D.1 Filament with hinged-hinged boundary conditions at the two tips . . 61

D.2 $\quad$ Filament with clamped-free boundary conditions at the two end tips. 63

D.3 Linear end to end distance in terms of $f_{m} \ldots \ldots$. . . . . . 64

D.4 Limit of large motor force $f_{m} \ldots \ldots \ldots \ldots$. . . . . . . . . 65

E The corelation Function of components of the tangent vector $\quad 67$

E.1 clamped-free case . . . . . . . . . . . . . . . . . 67

E.2 $\quad$ Hinged-hinged case . . . . . . . . . . . . . . . . . . . . 67

\begin{tabular}{ll}
\hline Bibliography & 71
\end{tabular}

\begin{tabular}{ll}
\hline Acknowledgement & 79
\end{tabular}

CV:

Mohammadhosein Razbin Khalilabad 


\section{Introduction}

Semiflexible filaments are abundant in biological materials. From cells to the extracellular environment, these kind of filaments are found. They are important for many biological organizations and processes. The maintenance and the change of a cell shape, the cell migration and development depend on the existence of cytoskeletal filaments. Also, the mechanical properties of the extracellular matrix play a role in cell migration and development and in other biological processes.

While most synthetic polymers are flexible chains, biopolymers fall into the category of semiflexible filaments. Biopolymers such as actin filaments, intermediate filaments and microtubules are categorized as semiflexible filaments. A flexible chain is a polymer which consists of orientationally uncorrelated links. The elastic energy required to bend such a chain is zero while it is nonzero for a semiflexible filament. A semiflexible filament has a defined structure and bending of it costs non-zero energy depending on the bending modulus of the filament. The main feature of biopolymers is defined by the persistence length at the single molecular level. The ratio of the persistence length to the contour length of the filament, $l_{p} / L$ characterizes how much the filament bends due to the thermal fluctuations. In fact the persistence length is the length scale on which the correlation of two tangent vectors at different location of the filament decreases by a factor of $1 / e$. If the ratio, $l_{p} / L$ is much smaller than 1, the filament is just a random coil and thermal fluctuations crumble the filament dramatically. If the ratio is larger than 1 , the filament is a semiflexible filament in the weakly bending regime which is the subject of interest in this thesis. In Chapter 2, we describe the approximations that are made for the study of semiflexible filaments in the weakly bending regime.

An actin filament is a double-stranded helical chain which is made up of actin monomers. After the formation of dimers and trimers of actin (nucleation process), the actin filament grows with a rate depending on the concentration of the actin monomers. Actin filaments are polar polymers with two ends called barbed end and pointed end. The barbed end grows about 10 times faster than the pointed end. The persistence length of an actin filament within a cell has not a unique value due to the presence of different types of interacting proteins but the order is $10 \mu \mathrm{m}$. Therefore any actin filament shorter than the cell size $\approx 10 \mu \mathrm{m}$ can be considered as a weakly bending semiflexible filament. The interaction of actin filaments with different proteins leads to the formation of different structures. Upon binding of passive crosslinkers to the actin filaments, the network of crosslinked actin filaments is formed. The interaction of these filaments with $A r p 2 / 3$ proteins results in the 
formation of a network of the branched actin filaments. The filaments can also make bundles with the help of a mixture of passive crosslinkers and myosin proteins.

The crawling of many different cell types is essential for life. Undifferentiated cells move towards the site, where they form a tissue or organ in the developing embryo. Skin cells start crawling when they have to close a wound [1]. During metastasis, cancer cells dissociate from the primary tumor, crawl towards blood vessels and spread all over the body [2, 3]. Branched actin filaments carry forces during cell motion, and consequently understanding their elastic properties is central to understanding the mechanics of cell motility. In vitro, cells are plated on a two dimensional substrate to observe their dynamics. They form flat membrane protrusions in the direction of motion, the lamellipodium, which is only about 100-200 nm thick but several $\mu \mathrm{m}$ deep and wide [4]. A dense network of actin filaments (F-actin) inside the lamellipodium pushes the leading edge membrane forward [5]. Treadmilling of the filaments drives motion [6]: The barbed (or plus) ends of the filaments polymerize at the leading edge of the lamellipodium and the pointed (or minus) ends depolymerize at the rear.Usually cells move in response to an external signal. A variety of signals stimulate the activation of nucleation promoting factors (NPFs) (like WASp or WAVE) located in the leading edge membrane of the lamellipodium. They activate the actin related protein complex Arp2/3. It binds to an existing filament very close to or at its barbed end at the lamellipodium's leading edge. That initiates the growth of a new filament branch out of the Arp2/3 complex. Many of these branched structures consisting of mother filament and daughter branch form the F-actin network in the lamellipodium. The branched structure itself is dynamic. The branch point with the Arp2/3 complex moves rearwards due to treadmilling in the same degree as mother filament and branch grow. Since Arp $2 / 3$ binding to the individual filaments is not synchronous we find many different positions of branch points in the lamellipodium F-actin network at any time. The elastic properties of the F-actin network crucially depend on the density of links between filaments [7, 8, 9, 10, 11, 12, 13. Molecular links arise in two ways: Cross-links connecting two filaments at some point along their contour length are formed by cross-linker molecules like filamin or $\alpha$-actinin, and branching attaches the minus end of a filament laterally to a mother filament. Intuition suggests that branching alone could stiffen the network to some degree, since branching is a geometrical constraint on the configuration of two filaments. That intuition has never been quantified before, but is supported by our results presented in Chapter 3. On the other hand, the network region close to the leading edge was found to be as soft as weakly cross-linked actin networks [9, 10, 11, 12, 13], and experiments in actin solutions suggest that branching contributes very little to the elastic modulus of F-actin networks [14]. In Chapter 3, we present a first step in quantifying the contribution of branching to the elastic and semi-flexible properties of the lamellipodial F-actin network. How much stiffer than the single filaments are the branched filaments? How are their properties reflected in network behav- 
ior? We will answer these questions by investigating a single branched filament and networks of branched structures in an approximation neglecting interactions between them in order to focus on branching effects. This neglect of interactions implies that we consider only elastic properties on short time scale and not the visco-elastic properties arising from cross-linking. The mother filament is grafted at one end and has a free tip at the other one in our model system (see Fig. 3.1). The graft is provided by a highly cross-linked part of the F-actin network. This idea is based on the increasing degree of cross-linking and filament bundling towards the rear of the lamellipodium, which has been observed in many different experiments and simulations [5, 15, 16, 17, 18, 19, 20, 21, 22, 23] (see [24] for a detailed discussion). The graft moves in the direction of cell motion due to cross-linker binding and bundling, and thus contour length 'flows' into the graft. In the steadily moving cell, the balance between polymerization and cross-linking creates a stationary distance between graft and leading edge membrane. At any time, the branched structure is in a configuration similar to Fig. 3.1, but the branch point moves with treadmilling towards the graft point. Both mother filament and branch polymerize such that their barbed ends stay at the leading edge membrane. Consequently, we will vary the position of the branch point on the mother filament from 0 to its full length $L$ when we investigate the elastic properties. We model the filaments in the weakly bending regime, i.e. bending does not affect the end-to-end distance. We allow for an elastic graft of stiffness $K_{s}$ and model the membrane by a constraint, enforcing the filaments to be entirely on the left side of the membrane.

As already mentioned, the basic structural elements of the cytoskeleton (microtubules, intermediate filaments, F-actin) are all semiflexible polymers with a behaviour intermediate between that of a random coil and a rigid rod [25, 26]. They form supramolecular assemblies (e.g. networks, bundles) through cross-linking [27]. Cross-linking involves a host of different filament-binding proteins [28, 29]. Active processes in the cell, such as the delivery of cargos, transport of organelles, mitotic dynamics, as well as muscle contraction are carried out by molecular motors using actin filaments or microtubules as tracks. The bottom-up approach to the study of molecular motors aims at analysing the transduction of metabolic energy into mechanical force and motion at the microscopic level using in vitro assays [30]. The advances in single-molecule manipulation are harnessed to study the simplest motor-filament complexes. In gliding assays, the motor (myosin, kinesin, or dynein) is attached to a glass surface and the translocation of the filament (F-actin or microtubule) is observed. In single motor assays, the filament is attached to the glass surface and the movement of the motor is monitored. In motor assays with beads, the motor is attached to a micron-sized refractile bead whose position is measured 31].

In Chapter 5, we investigate the mechanical response of a semiflexible filament with a discontinuous tension using analytical calculations. Our model system can be 
viewed as one of the simplest structural elements of the cytoskeleton beyond the isolated single-molecule level. We consider a semiflexible polymer, modelled as a wormlike chain, in the weakly bending approximation. The latter can be satisfied either by applying a strong tensile force which irons out large thermal undulations, or by having a filament with large persistence length compared to its contour length. A longitudinal Hookean spring whose one end is attached to a fixed substrate, has its other end on the filament thus exerting a force which causes a tension discontinuity. The longitudinal position of one end is held fixed, whereas that of the other end fluctuates. Its average position yields the force-extension relations which are the main subject of our analysis. The spring may be viewed as representing a motor, according to the myosin cross-bridge model first introduced by Huxley in 1957 [32] and still in use [33]. Our results apply to passive motors or to very slowly stepping motors, slower than the relaxation time of the filament. The time scale of the motor can be tuned by adjusting the concentration of ATP molecules. We should point out, however, that our study of semiflexible filaments with tension discontinuity is also relevant to passive cross-linkers of large size or compliance. Many of the actin binding proteins fall in this category as they can have large spacer domains [27].

In Chapter 4, We consider kinks in biopolymers in the weakly bending regime. A kink is one deflection in the filament which is defined by a rigid kink angle. Such kinks are found in structures like 30nm-chromatin fiber or DNA with induced kinks due to Cisplatin. Machanical properties of such structures are of interest in the field of biopolymers especially DNA mechanics.

All of the studies in this thesis are based on analytical calculations which are valid for filaments in the weakly bending regime. The contents of Chapter 3 and Chapter 5 is published in journals of physical biology and physical review E [34, 35]. 


\section{Wormlike chain model and weakly bending approximation}

\subsection{Wormlike chain model}

The wormlike chain model describes the statistical properties of an isotropic rod in the presence of thermal fluctuations. The rod is inextensible and semiflexible. The model is completely different than the freely jointed chain model in which the segments of the chain are connected in a flexible way and there is no energetic cost for bending the chain. There are several important biopolymers that can be effectively modeled as wormlike chains. DNA, RNA, actin filaments, intermediate filaments and microtubules are among the most important members of the list. In Fig. 2.1, the configuration of a free wormlike filament with its tangent vector and end to end vector is shown. Statistical properties of such a filament can be described by the following Hamiltonian [36],

$$
H=\frac{\kappa}{2} \int_{0}^{L} d s\left(\frac{\partial \vec{t}(s)}{\partial s}\right)^{2}
$$

Where $\kappa$ is the bending stiffness and $\vec{t}(s)=\frac{\partial \vec{r}(s)}{\partial s}$ is the tangent vector of the filament at contour parameter of $s$. The contour parameter is smaller than $L$ (contour length) and larger than zero. The filament is considered to be inextensible. This imposes an extra constraint to the Hamiltonian, and has the form

$$
|\vec{t}(s)|=1
$$

The average of the end to end distance of the filament is evaluated as,

$$
\langle\vec{R}\rangle=\int_{0}^{L}\langle\vec{t}(s)\rangle d s
$$

The tangent-tangent correlation function decays exponentially [37] as,

$$
\left\langle\vec{t}(s) \cdot \vec{t}\left(s^{\prime}\right)\right\rangle=\exp \left(-\frac{\left|s-s^{\prime}\right|}{l_{p}}\right)
$$




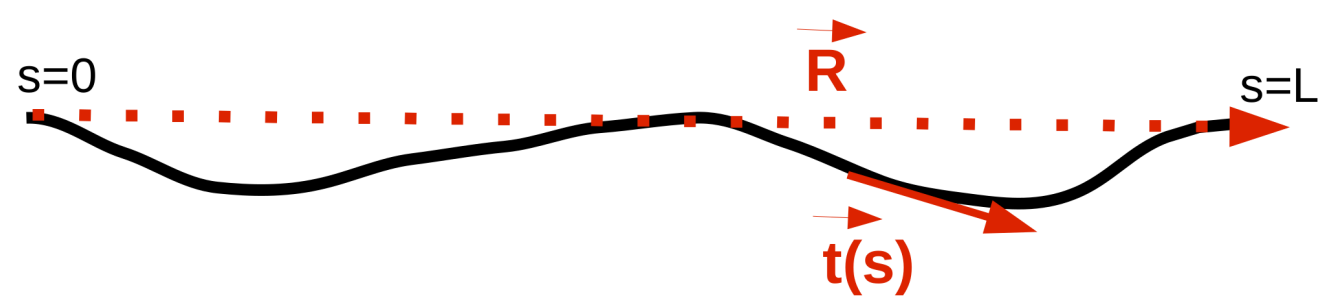

Figure 2.1: The configuration of a free semiflexible filament with the tangent vector $\vec{t}(s)$.

The persistence length $l_{p}=\frac{2 \kappa}{(d-1) k_{B} T}$ depends on the bending stiffness of the filament, the temperature and the dimension of the space in which the filament is placed. The mean-square end to end distance has the form [37]

$$
\left\langle R^{2}\right\rangle=\frac{2 L^{2}}{\epsilon^{2}}\left(\epsilon-1+e^{-\epsilon}\right)
$$

Where the ratio of the contour length to the persistence length of the filament is $\epsilon=\frac{L}{l_{p}}$. There are two interesting limiting case:

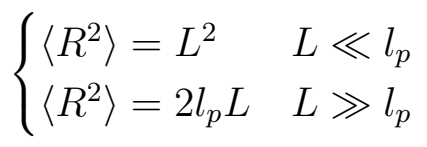

The case of $L \ll l_{p}$ and $L \gg l_{p}$ refer to the limit of the rigid rod and the Gaussian coil respectively.

\subsection{Weakly bending approximation}

In this thesis, we are dealing with the filaments in the weakly bending regime. The condition for the weakly bending approximation (WBA) can be satisfied by either keeping the persistence length of the filament large in comparison with the contour length or applying an external force. This section deals with two different approximations entering the calculations of the elasticity of filaments in the weakly bending regime. In the first subsection, we explain how the WBA enters the problems in Chapter 3 and Chapter 4. In the second subsection, we introduce the way in which the WBA enters the problems of Chapter 5 and Chapter 6. 


\subsubsection{Weakly bending approximation for calculation of the orientational-positional distribution function of semiflexible filament}

The orientational-positional distribution function of a two dimensional semiflexible filament is the probability to find the end tip of the filament at position $(x, y)$ with orientation angle $\theta$ (the orientation angle is the angle between the tangent vector of the filament and the $x$ axis) given that it is grafted at $\left(x_{0}, y_{0}\right)$ with orientation angle $\omega$. It can be obtained by solving the following partial differential equation [38]:

$$
\left[\frac{\partial}{\partial s}+\cos (\theta) \frac{\partial}{\partial x}+\sin (\theta) \frac{\partial}{\partial y}-\frac{1}{l_{p}} \frac{\partial^{2}}{\partial \theta^{2}}\right] G_{L}\left(x, y, \theta, s \mid x_{0}, y_{0}, \omega, 0\right)=0
$$

The orientation of the filament at graft point has been kept parallel to the $x$ axis. We employ the WBA by setting $\theta \ll 1$ which leads to $\sin (\theta) \approx \theta$ and $\cos (\theta) \approx 1$. Using this approximation and integrating over longitudinal coordinate $x$ the partial differential equation reduces to the form,

$$
\left[\frac{\partial}{\partial s}+\theta \frac{\partial}{\partial y}-\frac{1}{l_{p}} \frac{\partial^{2}}{\partial \theta^{2}}\right] G_{L}\left(y, \theta, s \mid y_{0}, 0,0\right)=0
$$

Solution of the PDE can be obtained by employing Fourier transformation and using the following initial condition [38],

$$
\lim _{s \rightarrow 0} G_{L}\left(y, \theta, s \mid y_{0}, 0,0\right)=\delta(\theta) \delta\left(y-y_{0}\right)
$$

it has the form [38],

$$
G_{L}\left(y, \theta, s \mid y_{0}, 0,0\right) \propto \exp \left[-\frac{3 l_{p}}{s^{3}}\left(\left(y-y_{0}\right)^{2}-L\left(y-y_{0}\right) \theta+\frac{s^{2} \theta^{2}}{3}\right)\right]
$$

\subsubsection{Weakly bending approximation in the problem of filament with a discontinuous tension}

Considering a filament embedded in three dimensional space, we choose a Mongelike parametrization for it. The tangent vector of the filament in the Monge-like parametrization has the form,

$$
t(s)=\left[\begin{array}{l}
t_{1}(s) \\
t_{2}(s) \\
t_{3}(s)
\end{array}\right]=\frac{1}{\sqrt{1+a_{1}^{2}(s)+a_{2}^{2}(s)}}\left[\begin{array}{c}
1 \\
a_{1}(s) \\
a_{2}(s)
\end{array}\right]
$$


For the filament in the weakly bending regime, the transverse components of the tangent vector $a_{1}(s)$ and $a_{2}(s)$ are small in comparison with the longitudinal one. Therefore the WBA is entering the problem by keeping all the expressions quadratic in terms of $a_{i}$. The components of the tangent vector are approximated as,

$$
\left\{\begin{array}{l}
t_{1}(s) \approx 1-\frac{1}{2}\left[a_{1}^{2}(s)+a_{2}^{2}(s)\right] \\
t_{2}(s) \approx a_{1}(s) \\
t_{3}(s) \approx a_{2}(s)
\end{array}\right.
$$

In this approximation, we can write $\left(\frac{d t(s)}{d s}\right)^{2} \approx \dot{a}_{1}^{2}(s)+\dot{a}_{2}^{2}(s)$ where the dot represents the derivative with respect to $s$. Therefore the Hamiltonian of a free wormlike filament has the form,

$$
H_{\mathrm{WBA}}=\sum_{i=1}^{2}\left[\int_{0}^{L}\left(\frac{\kappa}{2} \dot{a}_{i}^{2}(s)\right) d s\right]
$$

The average of the end to end distance in the longitudinal direction has the form,

$$
\left\langle R_{x}\right\rangle=L-\frac{1}{2} \sum_{i=1}^{2} \int_{o}^{L}\left\langle a_{i}^{2}(s)\right\rangle d s
$$




\section{Mechanical properties of branched actin filaments}

\subsection{The model: semiflexible branched filaments in the weakly bending limit}

Motile cells on a 2dimensional substrate generate motion by flat membrane protrusion called lamellipodia. As it is already mentioned in the introduction, lamellipodia is only about $100-200 \mathrm{~nm}$ thick but several $\mu \mathrm{m}$ deep. Within lamellipodia, actin filaments are generated by branching off existing ones, giving rise to branched network structures. We investigate the force-extension relation of branched actin filaments, grafted on an elastic substrate at one end and pushing with the free ends against a flat and stiff wall. We compute the thermal fluctuation of the endpoints and the resulting entropic forces on a membrane, restricting the fluctuations of the endpoints. We model the interior of the lamellipodium as a two-dimensional space, since it is approximately flat as described above. Furthermore, the results are easily generalised to three dimensions. We always assume a sufficiently large persistence length, such that the weakly bending approximation applies and the fluctuations perpendicular to the mean orientation of the polymer segment are small and can be treated on a Gaussian level.

Our elemental structure is a grafted filament of contour length $L$. The probability to find its tip at position $(x, y)$ with orientation $\theta$, given that it is grafted at $\left(x_{0}, y_{0}\right)$ with orientation $\omega$ is denoted by $G_{L}\left(x, y, \theta \mid\left(x_{0}, y_{0}, \omega\right)\right.$. For the simple case of perpendicular grafting, $\omega=0$, at $\left(x_{0}, y_{0}\right)=(0,0), G_{L}$ satisfies the following partial differential equation (PDE) [38]

$$
\left[\frac{\partial}{\partial s}+\theta \frac{\partial}{\partial y}-\frac{1}{l_{p}} \frac{\partial^{2}}{\partial \theta^{2}}\right] G\left(s, y, \theta \mid 0, y_{0}, 0\right)=0 .
$$

The arc length of the filament contour is denoted by $s$ here. The boundary condition

$$
\lim _{s \rightarrow 0} G\left(s, y_{s}, \theta_{s} \mid 0, y_{0}, 0\right)=\delta(\theta) \delta\left(y_{s}-y_{0}\right)
$$


realizes the graft. After a switch from the coordinates $(s, y, \theta)$ to $(x, y, \theta)$, the solution is 38

$$
G_{L}(x, y, \theta \mid 0,0,0) \propto \exp \left[-\frac{3 l_{p}}{L^{3}}\left(y^{2}-L y \theta+\frac{L^{2} \theta^{2}}{3}\right)\right] \delta(x-L) .
$$

The general case is obtained by a translation and rotation according to

$$
\begin{aligned}
& \theta \rightarrow \theta-\omega \\
& y \rightarrow\left(y-y_{0}\right) \cos \omega-\left(x-x_{0}\right) \sin \omega \\
& x \rightarrow\left(x-x_{0}\right) \cos \omega+\left(y-y_{0}\right) \sin \omega
\end{aligned}
$$

and explicitly is given by the following expression

$$
\begin{aligned}
& G_{L}\left(x, y, \theta \mid x_{0}, y_{0}, \omega\right) \propto \\
& \quad \exp \left[-\frac{3 l_{p}}{L^{3}}\left(\left(y-y_{0}\right) \cos (\omega)-\left(x-x_{0}\right) \sin (\omega)\right)^{2}\right. \\
& \left.\quad-\frac{l_{p}}{L}(\theta-\omega)^{2}\right] \\
& \times \exp \left[+\frac{3 l_{p}}{L^{2}}\left(\left(y-y_{0}\right) \cos (\omega)-\left(x-x_{0}\right) \sin (\omega)\right)(\theta-\omega)\right] \\
& \times \delta\left[\left(x-x_{0}\right) \cos (\omega)+\left(y-y_{0}\right) \sin (\omega)-L\right] .
\end{aligned}
$$

In a first step we compute the probability density, $P_{t}\left(x \mid x_{0}\right)$, that the endpoint of the tilted polymer is at a distance of $x-x_{0}$ from the graft point:

$$
P_{t}\left(x \mid x_{0}\right) \equiv \iint d y d \theta G_{L}\left(x, y, \theta \mid x_{0}, 0, \omega\right)
$$

We consider an elastic, fluctuating structure into which the filament is grafted and hence model it by a fluctuating spring in x-direction with spring constant $K_{s}=\left(k_{B} T\right) K$, zero equilibrium length and a distribution of the spring extension proportional to $\exp \left(-\left(K x_{0}^{2}\right) / 2\right)$. The stiffness of the substrate, $K$, is assumed to be large as compared to the stiffness for transverse fluctuations, $K \gg K_{\perp}=\frac{2 l_{p}}{3 L^{3}}$, of a filament of contour length $L$. In this study we take $K=100 \frac{2 l_{p}}{3(L \cos (\omega))^{3}}$. The probability $P_{t}(x)$ to find the filament tip at $x$ then follows by averaging over all graft point positions $x_{0}$ :

$$
P_{t}(x)=\int \frac{d x_{0}}{\sqrt{2 \pi K}} P_{t}\left(x \mid x_{0}\right) \exp \left(-\frac{K x_{0}^{2}}{2}\right) .
$$

The filament exerts a force on an impenetrable flat membrane at a distance $\delta$ from the graft plane (see Fig. 3.1). The force originates from the reduction in the number of filament configurations due to the constraint $x-x_{0} \leq \delta$ imposed by the membrane; 


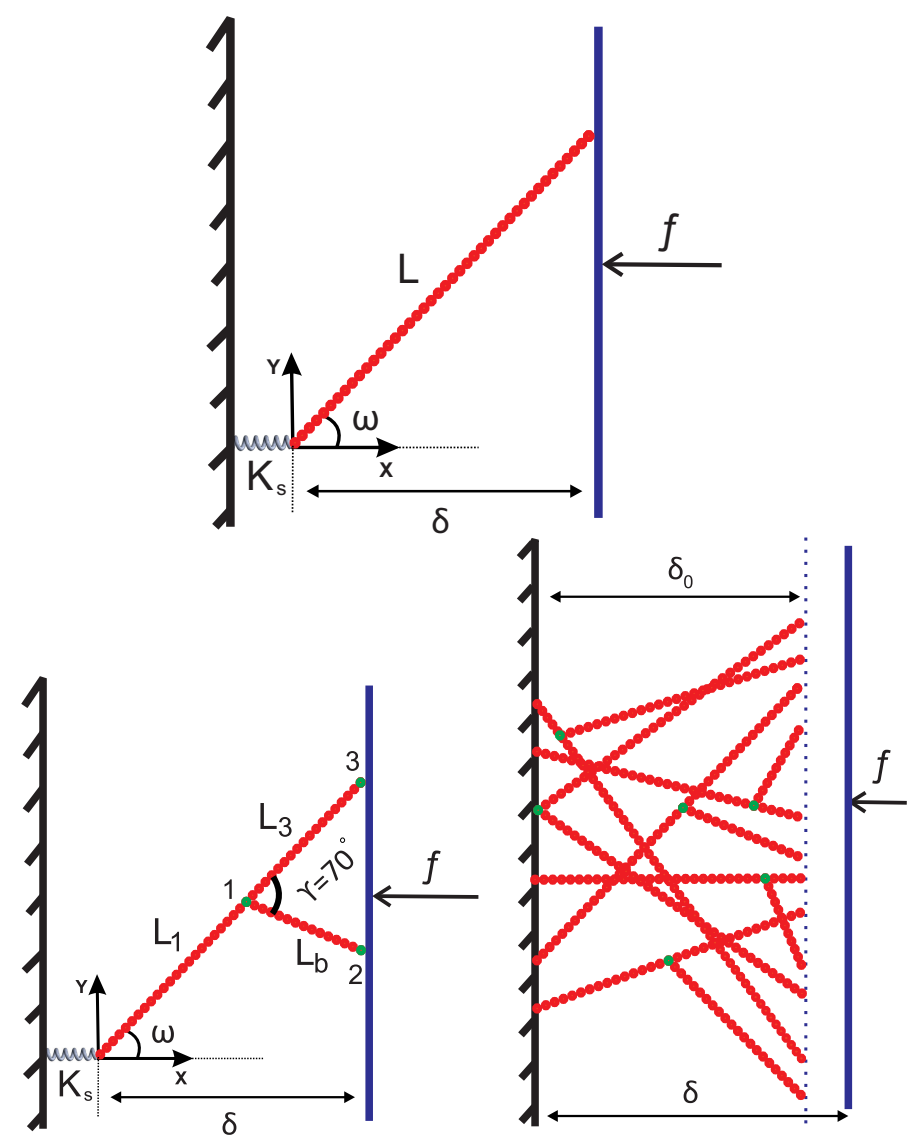

Figure 3.1: up: The single tilted polymer with orientation $\omega$ and length $L$ which is grafted on a substrate modeled by an spring with force constant $K_{s}$. Down Left: Single branched filament grafted on a soft graft and confined in the x-direction by a flat membrane. Thermal bending fluctuations are not shown in this drawing. $L_{1}$ is the contour length between graft point and branch point, $L_{3}$ is the contour length between branch point and filament tip. The contour length of the mother filament is $L=L_{1}+L_{3}, L_{b}$ is the branch contour length. The branch angle is $\gamma=70^{\circ}$ throughout the study, and $\omega$ is the tilt angle. The numbers 1, 2 and 3 refer to the filament tip coordinates in Eq. 3.10. Down Right: Network of branched filaments with various orientations and locations of branch points. The length $\delta_{0}$ is the distance of the filament tips from the graft plane without bending and fluctuations. The lengths $L$ and $L_{b}$ obey $L=\frac{\delta_{0}}{\cos (\omega)}, L_{b}=\frac{L_{3} \cos (\omega)}{\cos (\omega-\gamma)}$, and $\delta$ denotes the distance between the graft point and the leading edge membrane.

we therefore call it an entropic force. The fraction of configurations satisfying the constraint is given by

$$
Z_{t}(\delta)=\int d x P_{t}(x) \Theta(\delta-x)
$$


The derivative of $Z_{t}$ with respect to $\delta$ is the entropic force [36]

$$
f_{t}(L, \delta, \omega)=k_{B} T \frac{\partial}{\partial \delta} \ln Z_{t}(\delta)
$$

These results for a single grafted polymer are easily generalised to the branched structure shown in Fig. 3.1. The probability to find the two endpoints at $x_{2}$ and $x_{3}$ respectively given that the structure is grafted at point $x_{0}$ is calculated by the following expression:

$$
\begin{aligned}
& P_{b}\left(x_{2}, x_{3} \mid x_{0}\right)= \\
& \quad \iiint d x_{1} d y_{1} d \theta_{1} G_{L_{1}}\left(x_{1}, y_{1}, \theta_{1} \mid x_{0}, 0, \omega\right) \\
& \quad \times \iint d y_{2} d \theta_{2} G_{L_{b}}\left(x_{2}, y_{2}, \theta_{2} \mid x_{1}, y_{1}, \theta_{1}-\gamma\right) \\
& \quad \times \iint d y_{3} d \theta_{3} G_{L_{3}}\left(x_{3}, y_{3}, \theta_{3} \mid x_{1}, y_{1}, \theta_{1}\right)
\end{aligned}
$$

The stiffness of the graft is again taken into account by averaging over the positions of the graft:

$$
P_{b}\left(x_{2}, x_{3}\right) \propto \int d x_{0} P_{b}\left(x_{2}, x_{3} \mid x_{0}\right) \exp \left(-\frac{K x_{0}^{2}}{2}\right)
$$

The explicit expression for $P_{b}\left(x_{2}, x_{3}\right)$ is given by Eq. A.6 in the Appendix.A. The fraction of configurations satisfying the constraint obeys in analogy to Eq. 3.8

$$
Z_{b}(\delta)=\int d x_{2} \int d x_{3} P_{b}\left(x_{2}, x_{3}\right) \Theta\left(\delta-x_{2}\right) \Theta\left(\delta-x_{3}\right),
$$

and the entropic force follows from

$$
f_{b}\left(L, L_{b}, L_{3}, \delta, \omega\right)=k_{B} T \frac{\partial}{\partial \delta} \ln Z_{b}\left(L, L_{b}, L_{3}, \delta, \omega\right) .
$$

The force $f_{b}$ depends on the parameters $L \equiv\left(L_{1}+L_{3}\right), L_{3}, \omega$ and $\delta$ ( $L_{b}$ is fixed by $\omega, L$ and $\left.L_{3}\right)$. We will present our results as dimensionless quantities, and scale lengths by $\delta_{0}$ and force by $k_{B} T / \delta_{0}$ for that purpose. We comment on which specific values of parameters are suggested by experimental observations in the Discussion and the Appendix below.

\subsection{Properties of single branched filaments}

The entropic force exerted by the branched filament on the membrane is shown in the left panel of Fig. 3.2 for the symmetric case $\omega \sim 35^{\circ}$ and branching at the midpoint of the mother filament. The force decreases with increasing distance $\delta$ 

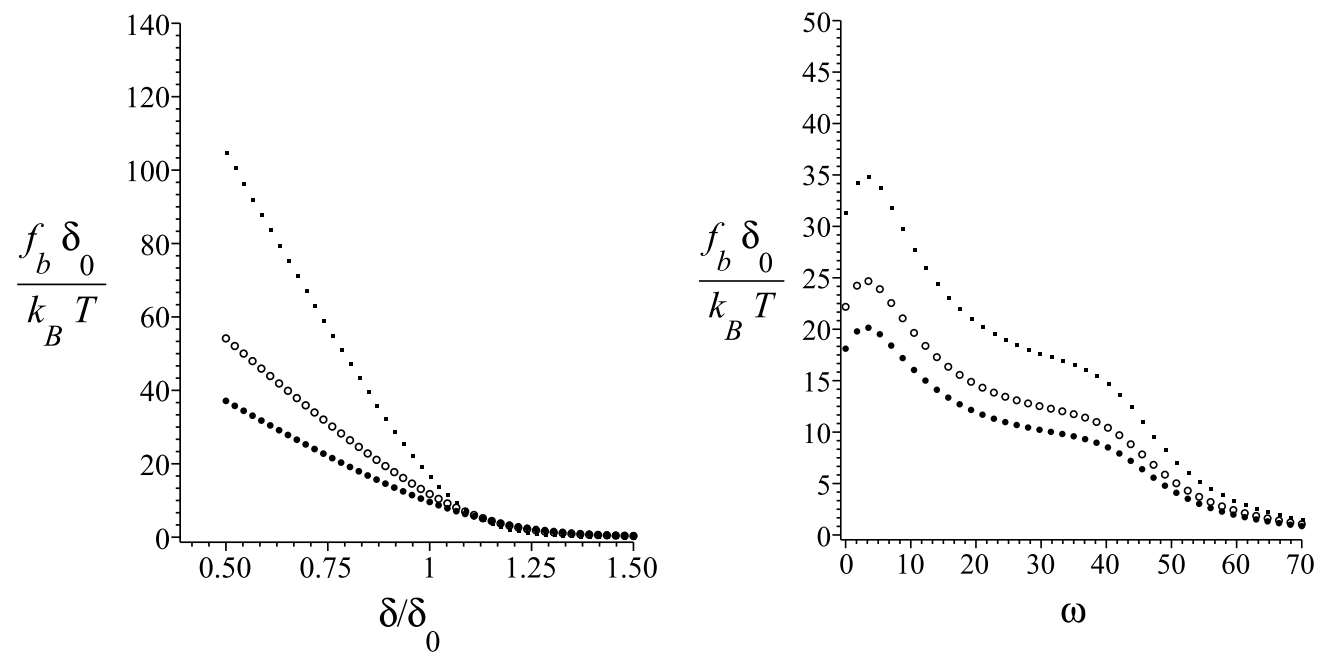

Figure 3.2: Left panel: force versus relative distance, $\delta / \delta_{0}$, between membrane and graft point for the symmetric case $\omega=35^{\circ}$ and $L_{3}=L_{b}=\frac{L}{2}$. Right panel: force versus tilt angle $\omega$ for $\delta=\delta_{0}$. The curves represent the ratio of $\frac{l_{p}}{\delta_{0}}=10,5$ and $\frac{10}{3}$ from top to bottom in both figures.

between grafting plane and membrane. An infinitely stiff filament $\left(l_{p}\right.$ very large) would just touch the membrane, if $\delta$ equals $\delta_{0}=L \cos \omega$. But the force exerted by a semiflexible filament is nonzero even for $\delta>\delta_{0}$, because the tilted branched structure exhibits fluctuations with the endpoint reaching beyond $\delta_{0}$. In this regime the force is nearly independent of persistence length, whereas for $\delta<\delta_{0}$ we observe a strong increase with $l_{p}$.

The force exerted by the filament on the membrane is crucially affected by the tilt angle. It is plotted as a function of tilt angle $\omega$ for fixed $\delta=\delta_{0}$ in the right panel of Fig. 3.2. Remarkably, there is a shoulder in all three curves around $\omega \sim 35^{\circ}$, indicating that the symmetric structure generates comparatively large forces. For $\omega \rightarrow 0$, the force would diverge for a stiff graft, since Eq. 3.3 excludes longitudinal fluctuations of the filament tip. The high but finite stiffness of the graft limits the force which can be exerted on the membrane. These two opposite effects generate the maximum in the force close to $\omega=0$.

Figure 3.3 shows the entropic force of a branched structure for the whole range of branch point positions on the mother filament. At the time of Arp2/3 binding to the mother filament $\left(L_{b}=0\right)$, the complete structure has of course the properties of the mother filament. When the branch point has reached the graft plane, the branched structure corresponds to two filaments with the corresponding tilt angles. In between, the force has a maximum at $L_{b}=0.484 L$. The maximum force is about 2.25 times the force of two filaments with length $L$. For the special case under consideration, $\delta=\delta_{0}$, the maximum of the force scales like the square root 

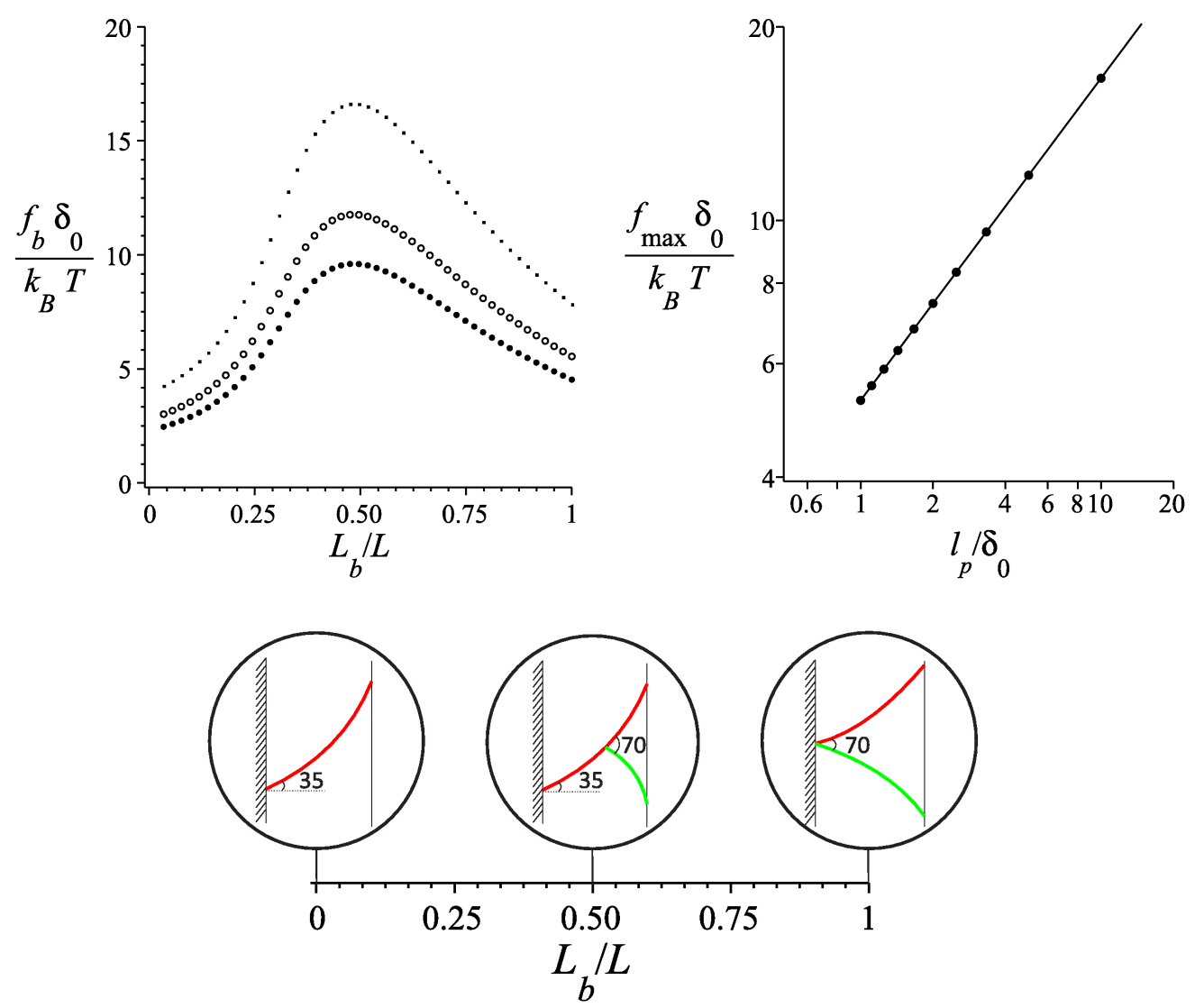

Figure 3.3: Left: force versus relative length of the daughter branch. Below: the configurations of the branched filament corresponding to $L_{b} / L=0,0.5,1.0$. The parameter values are: $L_{3}=L_{b}, \omega=35^{\circ}, \delta=\delta_{0}$. From high to low force values the symbols represent the ratio of $\frac{l_{p}}{\delta_{0}}=10,5$ and $\frac{10}{3}$, respectively. Right: $\log -\log$ plot of the maximum force as a function of persistence length $l_{p}$, fitted to a square root dependence $f_{\max } \propto \sqrt{l_{p}}$.

of $l_{p}$, as shown in the right panel of Fig. 3.3 . For the compressed case, $\delta<\delta_{0}$, we observe a crossover to linear scaling. To understand this behaviour of the force, we consider the case of a single filament: The force has a thermal (entropic) and a nonthermal (energetic) contribution. The thermal force dominates for $\delta \geqslant \delta_{0}$. Evaluating the force given in Eq A.5 for $\delta=\delta_{0}$ and large $K$, we explicitly see $f \propto \sqrt{l_{p}}$. As we compress the filament the energetic force becomes the dominant contribution. It can be computed as the force to bend a grafted cantilever beam resulting in $f=\left(\frac{3 l_{p} k_{B} T}{2 L^{3}}\right) \frac{(\delta-L \cos (\omega))}{\sin ^{2}(\omega)}$. Hence we observe a crossover from the scaling of the force with $\sqrt{l_{p}}$ to linear scaling as $\delta$ is decreased below $\delta_{0}$

Both, the maximum force, $f_{\max }$, as well as its branch point position, $L_{b}^{\max }$, depend on the tilt angle $\omega$. The general dependence on $\omega$ is shown in the top left panel of 

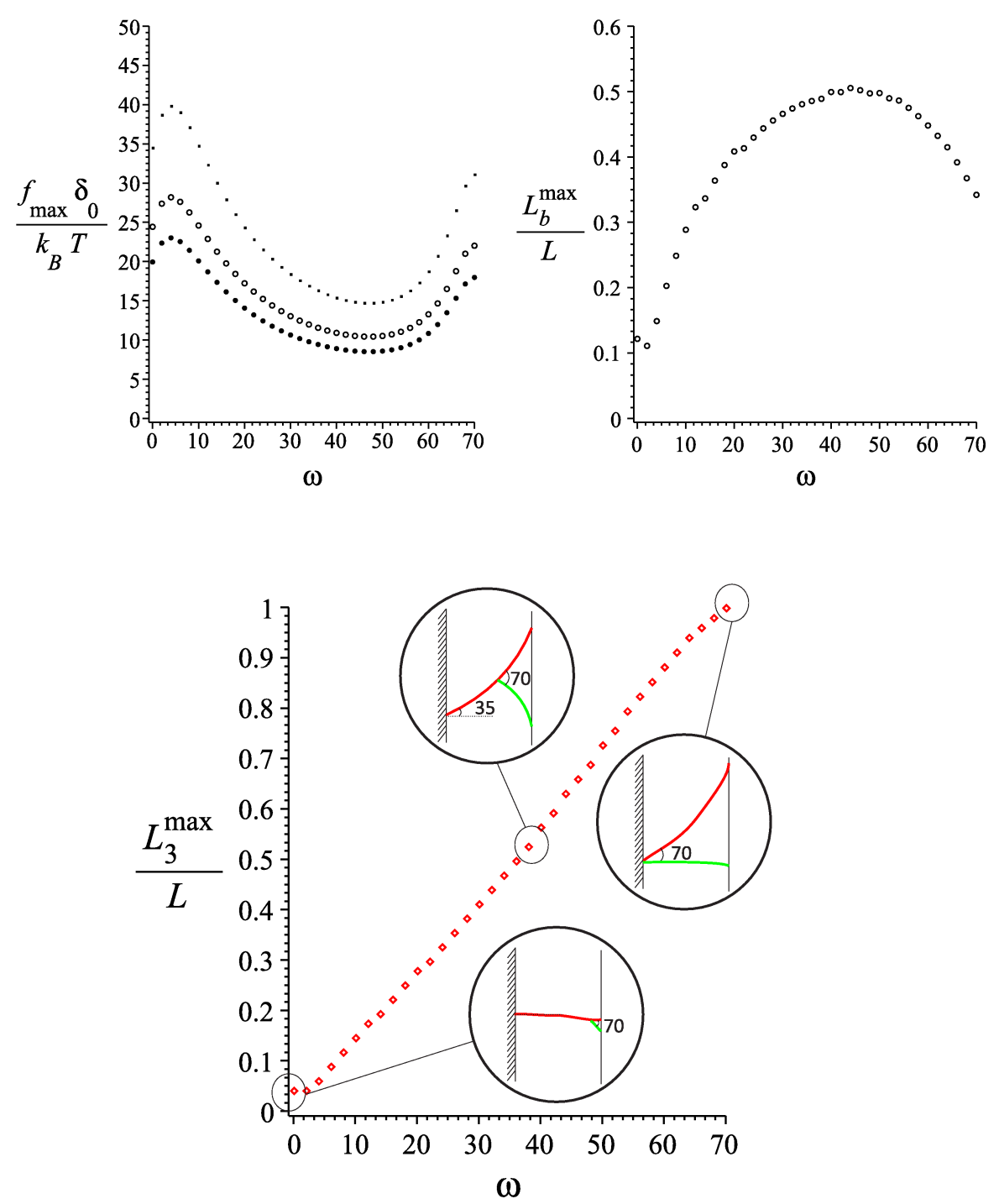

Figure 3.4: Upper left: Maximum of the force $f_{\max }$ obtained by varying $L_{b}$, versus tilt angle $\omega$; we have $\delta=\delta_{0}$ for the curves. From high to low force values the curves represent the ratio of $\frac{l_{p}}{\delta_{0}}=10,5$ and $\frac{10}{3}$, respectively. Upper right: Relative branch length $L_{b}^{\max } / L$ of the maximum force versus tilt angle $\omega$ for the same parameters (independent of the persistence length $l_{p}$ ). Bottom: Relative partial contour length of the mother filament in between the endpoint and the branch point with maximal force $L_{3}^{\max }=L_{b}^{\max } \cos (\omega-\gamma) / \cos \omega$; also shown are three representative configurations.

Fig. 3.4 for $f_{\max }$ and in the top right panel for $L_{b}^{\max }$. Since the dependencies are nonmonotonic, we plot in the bottom panel the configurations which give rise to the maximum force and help to understand the non-monotonic dependence. For very small $\omega$, i.e. almost perpendicular grafting, $L_{b}^{\max }$ is very small and hence also 
the distance between the branch point and the endpoint of the mother filament, denoted by $L_{3}^{\max }$. As the tilt angle, $\omega$, increases, the branch point moves further away from the leading edge membrane and hence both, $L_{b}^{\max }$ and $L_{3}^{\max }$ increase. As $\omega$ approaches $70^{\circ}$, the branch is almost perpendicular to the membrane and the maximum force is obtained for branching at the grafting plane, implying $L_{3}^{\max }=L$. Plotting $L_{b}^{\max } / L$ as in the top right panel of Fig. 3.4. one actually observes a decrease of $L_{b}^{\max } / L$, because $L$ grows faster than $L_{b}^{\max }$ as $\omega \rightarrow 70^{\circ}$.

$L_{3}^{\max }$ is independent of the persistence length, but does depend on $\delta$. For $\delta \geq$ $\delta_{0}$ entropic contributions dominate and the force is largest for two independently fluctuating filaments, such that $L_{3}^{\max } / L$ quickly approaches 1 as $\delta$ extends beyond $\delta_{0}$. On the other hand, for $\delta<\delta_{0}$, elastic contributions are important. In the symmetric case, we find $0.435 \leq L_{3}^{\max } / L \leq 0.484$ for $0.5 \leq \delta / \delta_{0} \leq 1.0$.

The maximum force is observed for $\omega \sim 0$ and $\omega \sim 70^{\circ}$, because either the mother filament or the daughter filament are perpendicular to the membrane (upper left panel of ). When the branch is perpendicular, the force is mainly determined by the fluctuations of the branch point, and when the mother filament is perpendicular by the fluctuations of the graft point. Moving away from perpendicular incidence the force has to decrease, giving rise to a minimum for intermediate $\omega$.

\subsection{Extension to the F-actin network: Properties of an ensemble of branched filaments}

We would like to obtain an estimate of how branching affects the network properties. With the theoretical means set up above and in the spirit of the study, we calculate the force as an average across an ensemble of branched structures, in which all interactions between the branched filaments are neglected. The ensemble is described by branch point $\left(L_{3}\right)$ and orientation $(\omega)$ distributions. The branch point is equally likely anywhere on the mother filament, corresponding to $0 \leq L_{3} \leq \delta_{0} / \cos \omega$. The length of the branch obeys $L_{b}=\frac{L_{3} \cos \omega}{\cos (\omega-\gamma)}$. As far as the orientation of the mother filaments is concerned, several scenarios have been discussed in the literature. In ref. [39], electron microscopy was used to determine the orientation of filaments in lamellipodial actin networks. The distribution was found to be approximately uniform in the range of angles between 0 and $60^{\circ}$ with a small contribution between between $60^{\circ}$ and $75^{\circ}$. We describe it here as a uniform distribution between $-70^{\circ}$ and $70^{\circ}$.

The force for fixed $\delta_{0}$ averaged over branch point positions and tilt angles is given by

$$
<f_{b}(\delta)>=\int_{\omega_{\min }}^{\omega_{\max }} d \omega \frac{\cos \omega}{\delta_{0} \Delta \omega}
$$



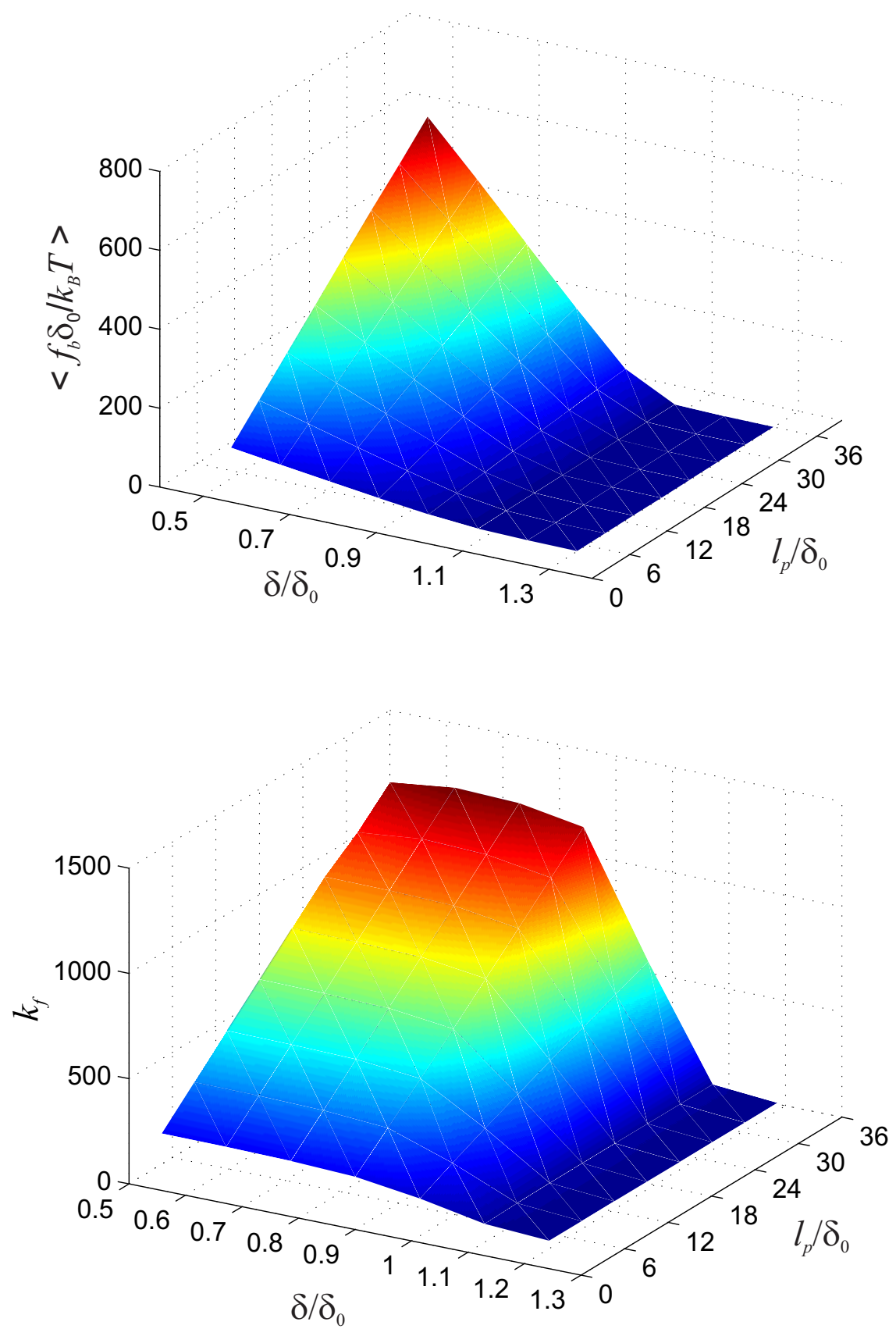

Figure 3.5: Up: Average force per branched structure of the network as a function of $\delta / \delta_{0}$ and $l_{p} / \delta_{0}$. Down: The force constant $k_{f}$, which is the derivative of $f_{b} \delta_{0} / k_{B} T$ with respect to $\delta / \delta_{0}$. 


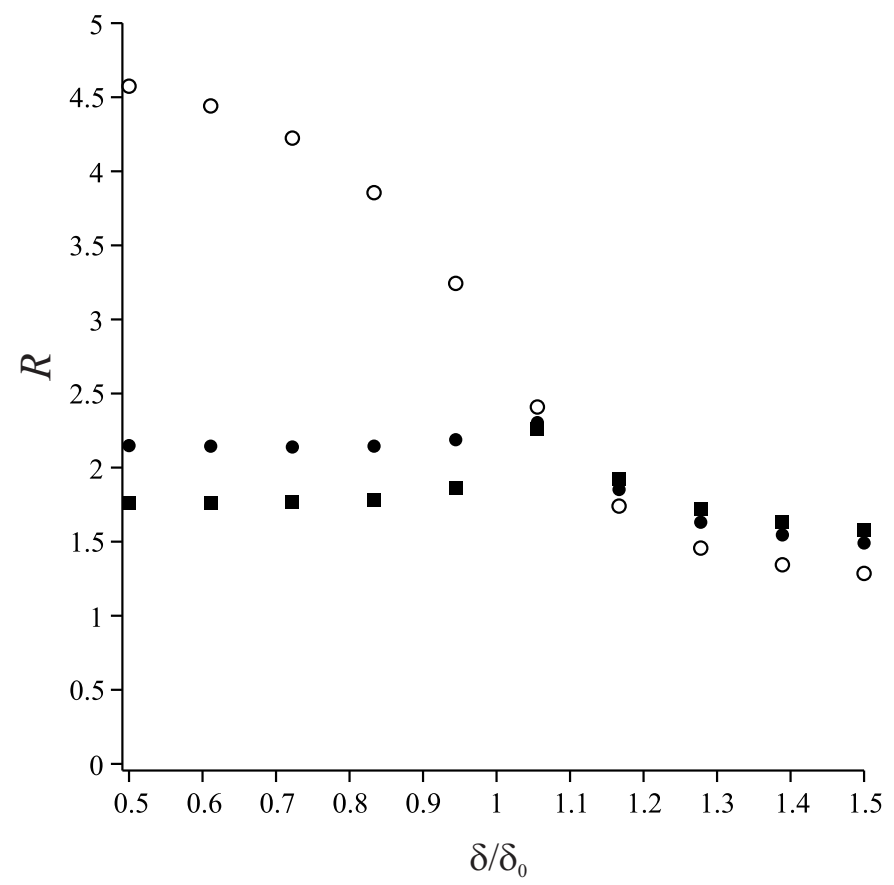

Figure 3.6: Ratio $R$ of the average force per filament of branched to unbranched networks versus relative distance $\delta / \delta_{0}$ between the membrane and the graft point; $\frac{l_{p}}{\delta_{0}}=10$. The squares refer to the random network with a uniform orientation distribution in the range $-70^{\circ} \leq \omega \leq 70^{\circ}$ as measured in ref. [39], the circles show results with a narrow uniform distribution of orientations in $30^{\circ} \leq \omega \leq 40^{\circ}$, the dots refer to the orientation distribution given by Eq. 3.16 as measured in ref. [40].

$$
\times \int_{0}^{\frac{\delta_{0}}{\cos \omega}} d L_{3} f_{b}\left(\frac{\delta_{0}}{\cos \omega}, \frac{L_{3} \cos \omega}{\cos (\omega-\gamma)}, L_{3}, \delta, \omega\right) .
$$

where $\Delta \omega=\omega_{\max }-\omega_{\min }$ denotes the range of the distribution. The average force is a monotonic function of both, the persistence length and the distance $\delta$ between grafting plane and membrane, as shown in Fig. 3.5.

We can compare our results with measurements of the force-velocity relation of fish keratocyte lamellipodia. The forces exerted by the leading edge of the freely running cell immediately upon collision with the cantilever were below the force resolution of the cantilevers [13, 11, 12, but caused an immediate decrease of leading edge velocity by 1-3 orders of magnitude. Hence, the leading edge is much softer than the softest cantilever used in the experiments, which had a force constant of 9.1 $\mathrm{nN} / \mu \mathrm{m}$. If the leading edge had the same force constant, a single branched structure would need to have a constant of about $11.8 \mathrm{pN} / \mu \mathrm{m}$ with 220 filaments $/ \mu \mathrm{m}$ [41] and a contact length of about $7 \mu \mathrm{m}[12]$. The value of $\delta_{0}$ in the freely running cell 
was estimated to be $\sim 1.3 \mu \mathrm{m}$ [13], and such a value is also supported by the data in ref. [42]. Hence, if the leading edge had the same force constant as the softest cantilever, the dimensionless force constant of a single branched structure would be

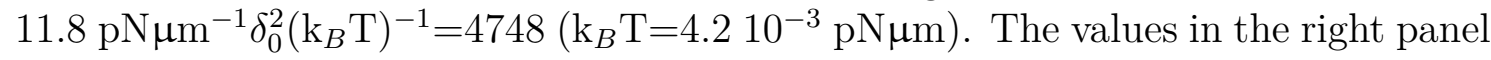
of Fig. 3.5 show that the branched filaments are much softer on average, which is in agreement with the dramatic velocity drop of the lamellipodium leading edge upon collision with the cantilever.

We do not know measurements of the pressure exerted by the filaments on the leading edge membrane in the freely running fish keratocyte but can estimate it from our results. Assuming $\delta_{0}=1.3 \mu \mathrm{m}, l_{p} / \delta_{0} \approx 10, \delta / \delta_{0} \approx 0.8$ [13] we obtain $f \delta_{0} / k_{B} T \approx$ 20 and a force per branched structure of $0.063 \mathrm{pN}$. The pressure exerted by the filaments on the leading edge membrane is in the range of $46 \mathrm{~Pa}$ (with 110 branched structures per micrometer and a lamellipodium height of $150 \mathrm{~nm}$ as in [12]).

The value of $\delta_{0}$ decreases during the force-velocity measurement due to a dynamic equilibrium between polymerization and cross-linking [13. In the stalled state, $\delta_{0} \approx 0.27 \mu \mathrm{m}$ applies (Fig. S3 of [13]). The measured stall pressure exerted on the leading edge by the cantilevers is $300-750 \mathrm{~Pa}$ [13] and 110-430 $\mathrm{Pa}$ [12]. These pressures correspond to stall forces of $0.045-0.1125 \mathrm{nN}$ [13] and $0.0165-0.0645 \mathrm{nN}$ [12] per micrometer leading edge. The values of $f \delta_{0} / k_{B} T$ resulting from these force densities are in the range 10-70. That entails $\delta / \delta_{0} \lesssim 1$ (see Fig. 3.5) in agreement with modelling results in ref. [13].

To assess the effects of branching on network properties, we calculate the ratio $R$ of the force exerted by a branched network to the force of an unbranched network with the same angular distribution. We use the same number of filaments in the unbranched network as there are branched structures in the branched network:

$$
R(\delta)=\frac{<f_{b}>}{\frac{1}{\Delta \omega} \int_{\omega_{\min }}^{\omega_{\max }} d \omega f_{t}\left(\delta_{0} / \cos \omega, \delta, \omega\right)}
$$

The ensemble averages of branched and unbranched networks with the uniform orientation distribution between $-70^{\circ}$ and $70^{\circ}$ behave very similar under compression (Fig. 3.6, squares). This ratio has the remarkable property of depending only very weakly on $\delta$. It is almost independent of the persistence length $l_{p}$ and the graft stiffness $K$ as well (data not shown). Hence, the behavior of both networks scales very similar in dependence on these parameters. The ratio is about 2 , i.e., the average force per filament tip for a given value of $\delta$ is the same for branched and unbranched networks.

However, there are obvious qualitative differences between single filaments and branched structures illustrated by the non-trivial dependency of the properties of branched structures on the branch point position in Figs. 3.3 and 3.4 . Indeed, if we use a narrow orientation distribution around $\omega=35^{\circ}$ the value of $R$ increases with increasing compression. Therefore, we also investigate non-uniform measured 
lamellipodial orientation distributions to investigate whether the dependency of $R$ on the distribution affects the behavior of lamellipodial networks. Distributions peaked either at $\omega=0$ or $\omega= \pm 35^{\circ}$ have been observed in refs. [40, 43, 44]. The distribution in ref. [40], their Fig. 4, is typical for the measured non-uniform distributions and can be approximated by

$$
\begin{gathered}
P(\omega)=0.008012\left[\mathrm{e}^{\frac{-(\omega-35)^{2}}{2 \cdot 20.5^{2}}}+\mathrm{e}^{\frac{-(\omega+35)^{2}}{2 \cdot 20.5^{2}}}\right] \\
+0.0006907, \quad-85^{\circ} \leq \omega \leq 85^{\circ}
\end{gathered}
$$

The dots in Fig. 3.6 show the results for $R$. They are very similar to the results for the uniform distribution. Obviously, the width of $40^{\circ}-45^{\circ}$ of the peaks in lamellipodial orientation distributions is too large for an essential effect of branching on the parameter dependencies.

\subsection{Summary}

We investigated the properties of branched filaments grafted into an elastic graft. Their stiffness has a maximum in its dependence on the branch point position. Branched structures with the optimal graft point position can be more than four times as stiff as a single filament with the same tilt angle as the mother filament (Fig. 3.3), while requiring only 1.5 times the polymer length of the mother filament.

The mechanical properties of branched F-actin networks depend on their orientation distribution. With measured lamellipodial distributions, networks of branched structures are about twice as stiff as unbranched networks. The total network force of branched networks scales essentially the same as the one of unbranched networks with the parameters F-actin persistence length, graft stiffness and compression $(\delta)$. An intuitive explanation would be, that in the end it is the single filament behaviour determining the stiffness for both unbranched and branched filaments, since the branch leans on the (single) mother filament when experiencing a force.

Our theory considers individual branched filaments and thus establishes the constitutive relations on which complex network studies including cross-linking can be based. We considered F-actin networks as defined by their geometrical property distributions without interactions of filaments by cross-linking or entanglement. This implies that we cannot account for visco-elastic properties. A variety of evidences suggests the existence of a region close to the leading edge, where cross-linking is not dominating the network properties and to which our theory directly applies. Measurements of the ratio of number of the cross-linkers to the number of actin molecules in fibroblasts show the existence of a gradient for $\alpha$-actinin and ABP$280 /$ filamin. The number ratios are low in a region juxtaposed to the leading edge with a depth of about $1.5 \mu \mathrm{m}$ (see Fig. 5 of ref. [15]). Svitkina and Borisy conclude 
from these results and structural information from electron micrographs that the impact of ABP-280 and $\alpha$-actinin on filament cross-linking is likely to be expressed more deeply in the cytoplasm [15]. Their statement is supported by the immediate response of the leading edge of fish keratocytes to small forces indicating also weak cross-linking in the network region close to the leading edge [13, 11, 12]. This suggests that while understanding of the visco-elastic properties of the network in the lamellipodium bulk requires taking cross-linking into account, our ensemble average is applicable to a network region close to the leading edge. The reproduction of both the weak and strong force responses of the lamellipodium leading edge measured in force-velocity relations by our network calculations strongly supports that conclusion (Fig. 3.5).

We did not take contributions from entanglement or excluded volume effects into account when calculating the network forces. This implies that $R$ provides only a meaningful approximation, if these effects are similar in branched and unbranched networks. To the best of our knowledge, that has not been investigated quantitatively yet. We can only provide heuristic considerations in favour of our assumption based on comparing a variety of simulations with and without excluded volume effects with force-velocity measurements.

Model networks of semi-flexible filaments not taking into account excluded volume effects reproduce the elastic properties measured in force-velocity experiments quantitatively [13]. Schreiber et al. simulated the force-velocity relation of motile cells with rigid rods as model filaments taking excluded volume effects into account [45. They found excluded volume effects to be stronger in branched than in unbranched systems. The model network of Schreiber et al. shows a response to external forces in the force-velocity relation at about $8 \mathrm{nN} / \mu \mathrm{m}$ [45]. However, the lamellipodium leading edge exhibits elastic responses to forces smaller than $0.05 \mathrm{nN} / \mu \mathrm{m}$ in experiments [13, 11, 12]. Additionally, bending of filaments has been observed in lamellipodia [39, 46, 47], i.e., filaments do not behave like stiff rods. Hence, the lamellipodium network is likely to be in a parameter regime where excluded volume effects are less relevant than suggested by a network of stiff rods.

Branching has also been observed with microtubule [48. The branching angle varies between $0^{\circ}$ and $90^{\circ}$, and it is not known, how rigid the connection to the mother filament is. If the branch is rigidly connected, our results should apply also to these branched structures with the adapted persistence length (a few millimeters [49]) and $\gamma$-values. 



\section{Mechanical properties of Filament with kinks}

Kinks in filaments of biopolymers can be found in many cases. One very nice example is 30nm-chromatin fiber which has a zig-zag shape and it consists of several links of DNA attached by nucleosomes. This structure is one of the main phase of DNA polymer which appears in the process of DNA compaction into chromatin. This process dramatically reduces the DNA length (10000 fold reduction) [50]. Also, the Cisplatin is one of the popular antitumor drugs and its function is to induce a kink in DNA by an angle $\gamma=40$ which finally leads to cell death in cancer cells [51. Another example is the Sorona 3GT polymer which has a zigzag molecular shape and can be seen as one straight polymer with regular kinks [52]. For these examples, a model for the description of the elasticity of such structures is needed on the molecular level. We obtain analytical results based on the Gaussian wormlike chain model. The model describes both entropic and elastic properties of such kinked filaments when the persistence length is larger than the length scale of the structure. We have calculated the probability density distribution of the end point of the structure and therefore it becomes possible to calculate the entropic and elastic force exerted by the structure.

\subsection{The probability of finding the end tip of the filament with one kink at fixed position}

In order to calculate the probability of finding the end tip of the kinked polymer at position $x_{2}$, we use the Gaussian propagator for the single filament (see Eq. 3.5). The Gaussian propagator captures the mechanical properties of the filament in the weakly bending approximation. It gives a good estimate of the filament's mechanical properties when the persistence length is larger than the length scale of the structure. With the Gaussian propagator, we can calculate the probability density function of the position of the end tip of the kinked filament in the $x$ direction. It has the form,

$P\left(x_{2}\right)=\iiint d x_{1} d y_{1} d \theta_{1} G_{L_{1}}\left(x_{1}, y_{1}, \theta_{1} \mid 0,0, \omega\right)\left[\iint d y_{2} d \theta_{2} G_{L_{2}}\left(x_{2}, y_{2}, \theta_{2} \mid x_{1}, y_{1}, \theta_{1}+\gamma\right)\right]$ 
The integration of $G_{L_{2}}\left(x_{2}, y_{2}, \theta_{2} \mid x_{1}, y_{1}, \theta_{1}+\gamma\right)$ over $\theta_{2}$ and $y_{2}$ gives the following expression:

$$
\begin{aligned}
& \int G_{2}\left(x_{2}, y_{2}, \theta_{2} \mid x_{1}, y_{1}, \theta_{1}\right) d y_{2} d \theta_{2} \propto \exp \left(-\frac{3 l_{p}}{4 L_{2}} \theta_{1}^{* 2}\right) \\
& \quad \times \exp \left(-\frac{3 l_{p}}{2 L_{2}^{2}} \frac{\left(x_{2}-x_{1}-L_{2} \cos (\omega+\gamma)\right)}{\sin (\omega+\gamma)} \theta_{1}^{*}-\frac{3 l_{p}}{4 L_{2}^{3}} \frac{\left(x_{2}-x_{1}-L_{2} \cos (\omega+\gamma)\right)^{2}}{\sin ^{2}(\omega+\gamma)}\right)
\end{aligned}
$$

Where $\theta_{1}^{*} \equiv\left(\theta_{1}-\omega\right), L_{1}$ is the length of the first segment of the kinked filament, $L_{2}$ is the length of the second segment and $l_{p}$ is the persistence length (see Fig. 4.1). The integration over the remaining parameters in Eq. 4.2 leads to the probability density function of the position of the end tip of the filament with one kink which has the form,

$$
P\left(x_{2}\right) \propto \exp \left(-\frac{\left(x_{2}-L_{1} \cos (\omega)-L_{2} \cos (\omega+\gamma)\right)^{2}}{\sigma_{k x}^{2}}\right)
$$

Where:

$$
\begin{aligned}
\sigma_{k x}^{2}= & \frac{4}{3 l_{p}}\left[L_{2}^{3}+3 L_{1} L_{2}^{2}\right] \sin (\omega+\gamma)^{2}+\frac{12}{3 l_{p}} L_{1}^{2} L_{2} \sin (\omega) \sin (\omega+\gamma) \\
& +\frac{4}{3 l_{p}} L_{1}^{3} \sin (\omega)^{2}
\end{aligned}
$$

The probability density function is a Gaussian expression (as it was expected) and it reproduces the probability density function of the end tip of the single filament with length $L$ when $\gamma=0$ and $L_{1}=L_{2}=\frac{L}{2}$.

Another quantity which is of interest is the probability density function of the position of the end tip of the kinked filament in $y$ direction. It has the form,

$$
P\left(y_{2}\right)=\iiint d x_{1} d y_{1} d \theta_{1} G_{L_{1}}\left(x_{1}, y_{1}, \theta_{1} \mid 0,0, \omega\right)\left[\iint d x_{2} d \theta_{2} G_{L_{2}}\left(x_{2}, y_{2}, \theta_{2} \mid x_{1}, y_{1}, \theta_{1}+\gamma\right)\right]
$$

After integration, we end up with the following expression for the probability density function,

$$
P\left(y_{2}\right) \propto \exp \left(-\frac{\left(y_{2}-L_{1} \sin (\omega)-L_{2} \sin (\omega+\gamma)\right)^{2}}{\sigma_{k y}{ }^{2}}\right)
$$

Where:

$$
\begin{aligned}
\sigma_{k y}^{2}= & \frac{4}{3 l_{p}}\left[L_{2}^{3}+3 L_{1} L_{2}^{2}\right] \cos (\omega+\gamma)^{2}+\frac{12}{3 l_{p}} L_{1}^{2} L_{2} \cos (\omega) \cos (\omega+\gamma) \\
& +\frac{4}{3 l_{p}} L_{1}^{3} \cos (\omega)^{2}
\end{aligned}
$$




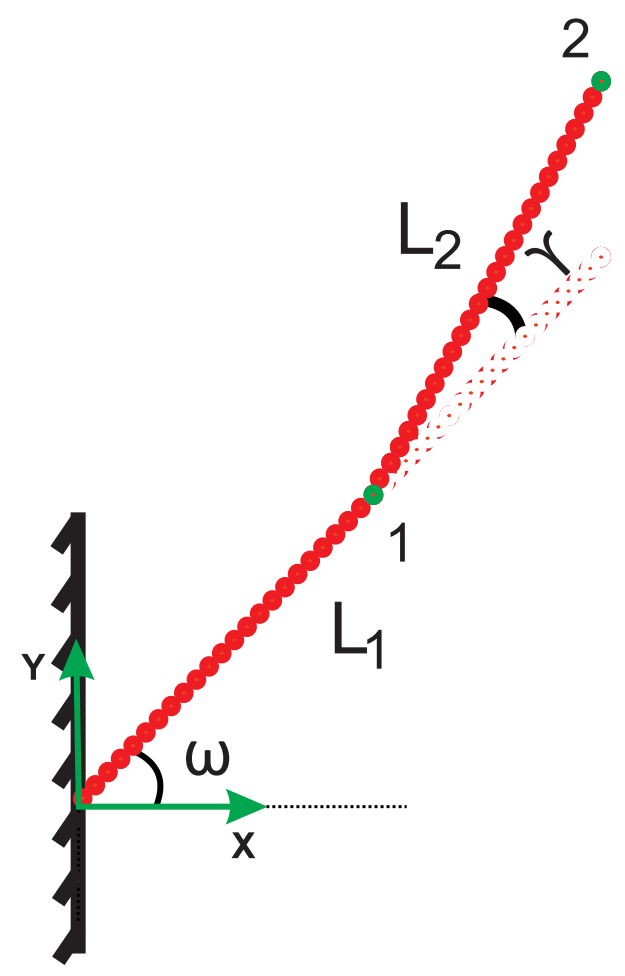

Figure 4.1: The zero temperature configuration of the filament with one kink grafted on a substrate.

\subsection{The probability density function of the regularly kinked filament}

In this section, we describe the probability density function (PDF) of the position of the end tip of the filament with regular kinks. The results is obtained by using the Gaussian propagator of one filament and mathematical induction. The probability density function of the position of the end tip of the filament with a number $m$ of segments in $x$ coordinate is explicitly given by

$$
P\left(x_{m}\right) \propto \exp \left(-\frac{3}{4} \frac{l_{p} \eta_{x}^{2}}{m L^{3} \sin (\omega)^{2}}\right)
$$

and the expression for the probability density function of the position of end tip of the filament with $m$ number of segments in $y$ coordinate $P\left(y_{m}\right)$ has the form,

$$
P\left(y_{m}\right) \propto \exp \left(-\frac{3}{4} \frac{l_{p} \eta_{y}^{2}}{m^{3} L^{3} \cos (\omega)^{2}}\right)
$$




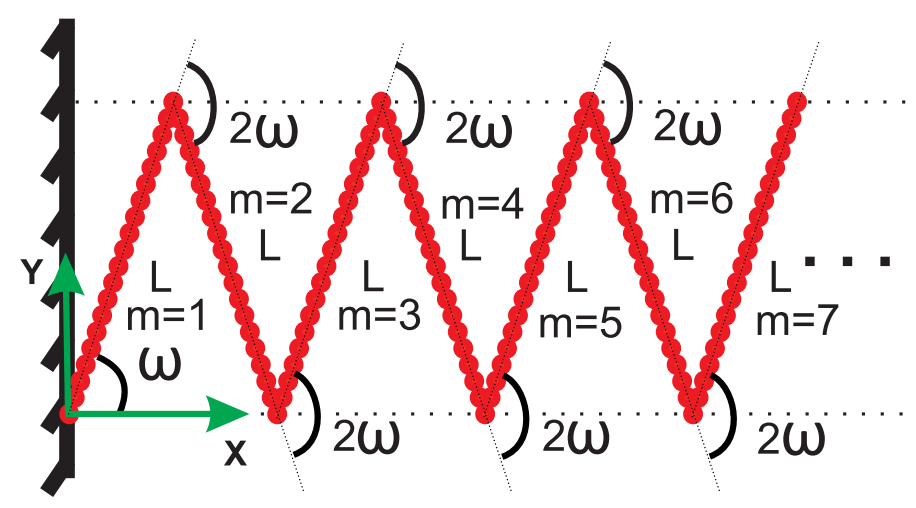

Figure 4.2: The zero temperature configuration of the filament with regular kinks grafted on a substrate.

Where we have defined $\eta_{x}=x_{m}-m L \cos (\omega)$ and $\eta_{y}=y_{m}-\left(\frac{1-(-1)^{m}}{2}\right) L \sin (\omega)$ and $x_{m}$ and $y_{m}$ are the components of the position of the end tip of the segment $m$. The proof for obtaining the expressions can be found in appendix.B .

\subsection{The force-extension relation in the fixed extension ensemble}

The force-extension relation in the fixed extension ensemble can be obtained by fixing the extension of the end point of the filament at $x$ and measuring the force and calculating the average value of it. The probability density function of the position of the end tip of the filament in $x$ coordinate is:

$$
P(x)=\left\langle\delta\left(\left|r_{x}(L)\right|-x\right)\right\rangle=\int D\{\vec{r}(s)\} \exp (-\beta H) \delta\left(\left|r_{x}(L)\right|-x\right)
$$

Where $r_{x}(L)$ is the $x$ component of the end to end distance of the filament, $\beta=\frac{1}{k_{B} T}$ and $H=\frac{\kappa}{2} \int_{0}^{L} d s\left(\frac{\partial \vec{t}(s)}{\partial s}\right)^{2}$ is the Hamiltonian of the filament. $P(x)$ is just the partition function of the filament in the fixed extension ensemble, with $r_{x}(L)=x$. The partition function defines the free energy as,

$$
F_{x}(x)=-k_{B} T \ln (P(x))
$$

Therefore the first moment of the force in the $x$ direction has the following relation with $P(x)$ :

$$
\left\langle f_{x}\right\rangle_{r_{x}(L)=x}(x)=\frac{1}{\beta} \frac{\partial}{\partial x} \ln (P(x))
$$


We call this relation the force-extension relation of the filament in the fixed extension ensemble.

Similar reasoning leads to the force-extension relation of the filament in the $y$ direction:

$$
\left\langle f_{y}\right\rangle_{r_{y}(L)=y}(y)=\frac{1}{\beta} \frac{\partial}{\partial y} \ln (P(y))
$$

Where $f_{y}$ is the force exerted on the filament in the $y$ direction.

\subsection{The force exerted on a restricting wall}

Similar to the previous chapter, we put a flat and stiff wall in front of the filament. The presence of the wall reduces the number of configurations of the filament and the wall experiences a fluctuating force. The force on the wall is obtained from the following relation which is also described in Chapter 3:

$$
\left\langle f_{x}\right\rangle(\delta)=k_{b} T \frac{\partial}{\partial \delta} \ln Z(\delta)
$$

Where the fraction of configurations $Z(\delta)$ is given by

$$
Z(\delta)=\int d x P(x) \Theta(\delta-x) .
$$

Instead of fixing the position of the end tip of the filament in $x$, we sum over all configuration of the filament with $x<\delta$ and calculate the average of the entropic force.

\subsection{The force associated with the filament with one kink}

With the probability density function of the position of the end tip of the filament with one kink in Eq. 4.3 , the force-extension relation has the form:

$$
\left\langle f_{x}\right\rangle\left(x_{2}\right)=-\left(\frac{2 k_{B} T}{\sigma_{k x}^{2}}\right)\left(x_{2}-L_{1} \cos (\omega)-L_{2} \cos (\omega+\gamma)\right)
$$

$\sigma_{k x}$ is calculated in Eq. 4.4. The force-extension relation is purely elastic.

The average of the force on the wall for the system of the filament with one kink and the flat wall attains the form:

$$
\left\langle f_{x}\right\rangle(\delta)=\left(\frac{2 k_{B} T}{\sqrt{\pi} \sigma_{k}}\right) \frac{\exp \left(-\frac{\left(\delta-L_{1} \cos (\omega)-L_{2} \cos (\omega+\gamma)\right)^{2}}{\sigma_{k}{ }^{2}}\right)}{\operatorname{erfc}\left(\frac{-\delta+L_{1} \cos (\omega)+L_{2} \cos (\omega+\gamma)}{\sigma_{k}}\right)}
$$




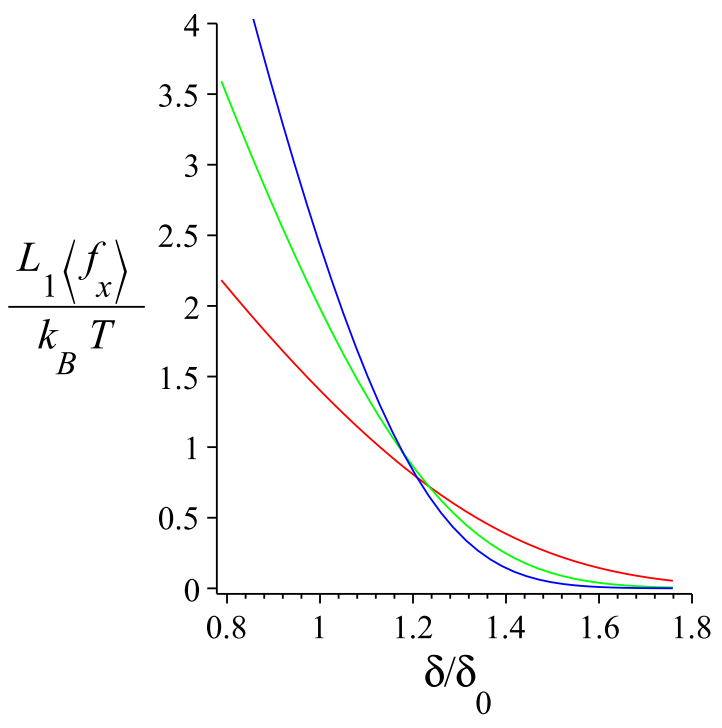

Figure 4.3: The average of the force on the wall is shown for the filament with one kink. The given parameters are as follows: $L_{1}=1 \mu m, L_{2}=1 \mu m, \omega=\gamma=20^{\circ}$. The red, green and blue are coresponding to $l_{p}=5 \mu \mathrm{m}, 10 \mu \mathrm{m}, 15 \mu \mathrm{m}$ respectively.

In Fig. 4.3 , we plot the force on the wall for the system of wall and the kinked filament with three values of the persistence length. The force is higher for the higher persistence length when the filament is compressed by the wall and it is opposite when the wall is far away from the position of endpoint of the filament at zero temperature. Since the force is dominated by the elastic part in the compressed case, we see larger force for the higher value of the persistence length. When the distance of the wall $\delta$ is further away from the endpoint of the filament at $T=0$, the entropic contribution dominates the force. In this case, softer filaments can fluctuate and hit the wall easier than stiffer ones therefore the more softer the filament the more force it exerts on the wall.

In Fig. 4.4, the force as a function of orientation angle $\omega$ is shown for different kink angle $\gamma$. The curves show a maximum at an orientation angle $\omega_{\max }$ which depends on the value of the kink angle. The orientation angle $\omega_{\max }$ increases while the kink angle decreases. The value of maximum of force also depends on absolute value of the kink angle, it increases while the kink angle decreases towards zero where the maximum of force is not well defined or is infinite.

\subsection{The force exerted by the regularly kinked filament}

The probability density function of the position of the end tip of the filament with regular kinks in Eq. 4.8 and Eq. 4.9 gives the following result for the components $(x$ 


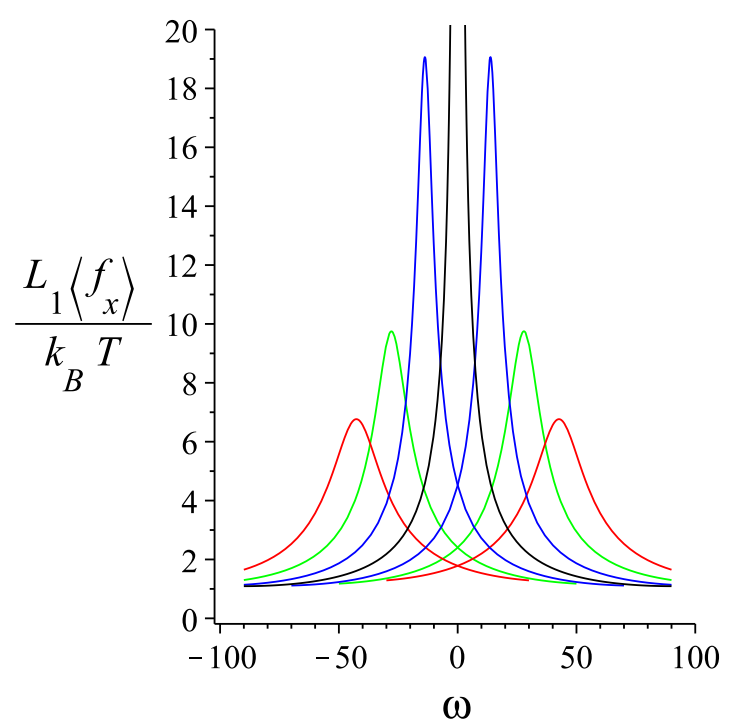

Figure 4.4: The average of the force on the wall versus the orientation angle at grafting point $\omega$ is shown for the filament with one kink. The given parameters are as follows: $L_{1}=1 \mu m, L_{1}=1 \mu m, l_{p}=10 \mu m, \delta=L_{1} \cos (\omega)+L_{2} \cos (\omega+\gamma)$. The red, green, blue and black are coresponding to $\gamma= \pm 60^{\circ}, \gamma= \pm 40^{\circ}, \gamma= \pm 20^{\circ}$ and $\gamma=0^{\circ}$ respectively. The curves with peak on the right side of axis $\omega$ are coresponding to minus sign of value of $\gamma$ and the one with peak on the left side are coresponding to plus sign.

and $y$ components) of the force-extension relation:

$$
\left\langle f_{x}\right\rangle\left(x_{m}\right)=-\left(\frac{3 \kappa}{m L^{3} \sin (\omega)^{2}}\right)\left(x_{m}-m L \cos (\omega)\right)
$$

and

$$
\left\langle f_{y}\right\rangle\left(y_{m}\right)=-\frac{3 \kappa}{m^{3} L^{3} \cos (\omega)^{2}}\left(y_{m}-\left(\frac{1-(-1)^{m}}{2}\right) L \sin (\omega)\right)
$$

Similar to Sec. 4.5, the two force-extensions are purely elastic.

And the average of the force on the wall has the form:

$$
\left\langle f_{x}\right\rangle(\delta)=\left(\frac{k_{B} T}{\sin (\omega) \sqrt{\frac{\pi m L^{3}}{3 l_{p}}}}\right) \frac{\exp \left[-\left(\frac{3 l_{p}}{4 m L^{3}}\right)\left(\frac{\delta-m L \cos (\omega)}{\sin (\omega)}\right)^{2}\right]}{\operatorname{erfc}\left[\left(\frac{3 l_{p}}{4 m L^{3}}\right)^{\frac{1}{2}}\left(\frac{m L \cos (\omega)-\delta}{\sin (\omega)}\right)\right]}
$$

In Fig. 4.5, the $x$ component of the force on the wall for the system of the filament with regular kinks and the wall is shown for different values of $\omega$. The curves show that the force in the $x$ direction is higher for the smaller value of $\omega$ therefore the 


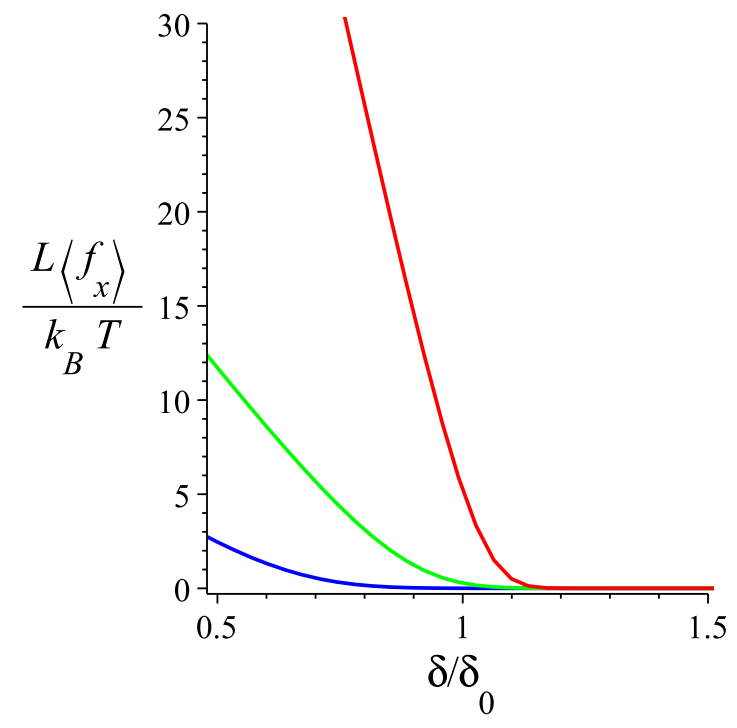

Figure 4.5: The average of the force on the wall is shown for the filament with regular kinks. The given parameters are as follows: $L=1 \mu m, l_{p}=10 \mu m, m=3$. The red, green and blue are coresponding to $\omega=20^{\circ}, 40^{\circ}, 60^{\circ}$ respectively.

filament is stiffer in the $x$ direction when the angle $\omega$ is smaller. This is the same for the $x$ component of the force in the fixed extension ensemble and it can be seen from Eq. 4.18

The average of the force in the $x$ direction for the fixed extension ensemble is proportional to $1 / m$ while the force in the $y$ direction is proportional to $1 / \mathrm{m}^{3}$. In the $x$ direction, the filament looks like a system consisting of $m$ springs in series each of which with spring constant $\frac{3 \kappa}{L^{3} \sin ^{2}(\omega)}$.

In Fig. 4.6, we show the dependency of the force constant in the $x$ direction on the number of segments of the regularly kinked filament. As we increase the number of segments the filament becomes softer. The black dashed line is the elastic force constant or force constant in the fixed extension ensemble and the lines are the force constant of the wall. The different colors of lines corespond to the different degrees of compression by the wall. The force constant of the wall is always less than the elastic force constant and it converges to the elastic force constant as we compress the filament by the wall. This non-linear behaviour originates from the integration of the probability density function of the position of the end tip of the filament which is a Gaussian distribution (see Eq. 4.15). 

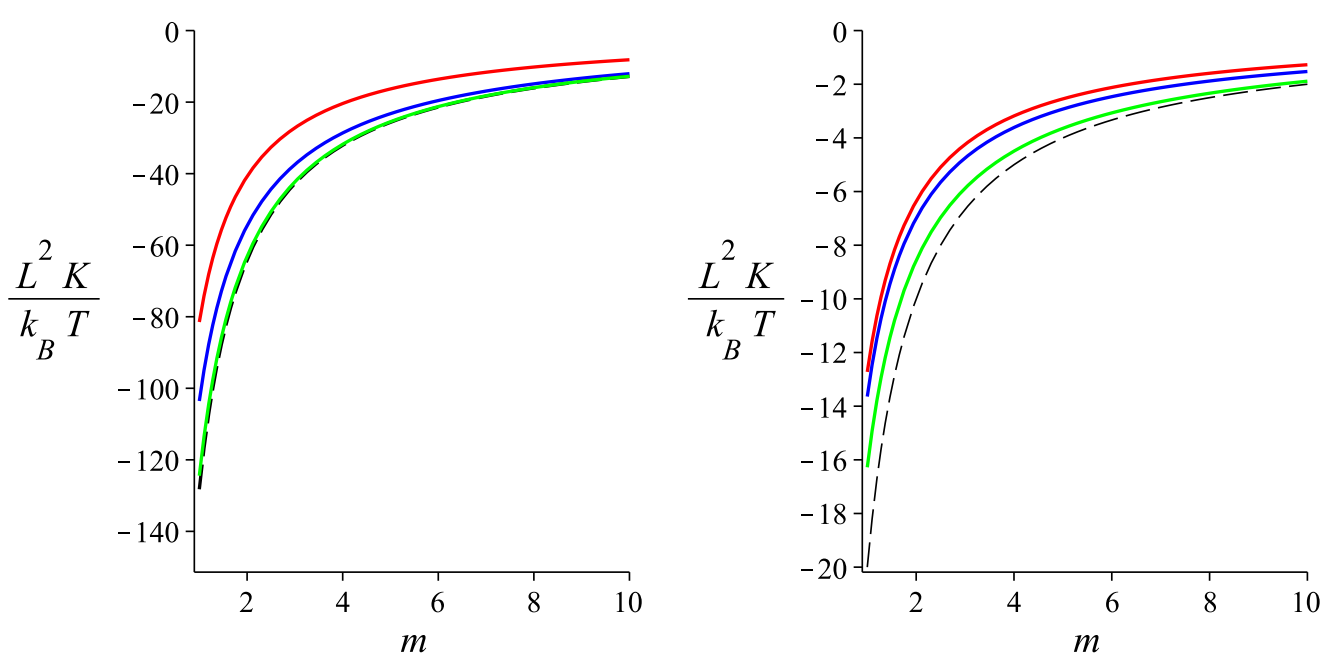

Figure 4.6: The force constant $K$ (the force constant is $\frac{\partial\left\langle f_{x}\right\rangle}{\partial \delta}$ for the system of filament and the wall and it is $\frac{\partial\left\langle f_{x}\right\rangle}{\partial x_{m}}$ for the filament in the fixed extension ensemble.) as a function of number of the segments is shown for the parameters: $L=1 \mu \mathrm{m}, l_{p}=$ $10 \mu \mathrm{m}$ the black dashed line is the elastic force constant or force constant at the fixed extension ensemble and the lines are the force constant of the wall. The red, blue and green colors are coresponding to $\delta=\delta_{0}, \delta=0.9 \delta_{0}, \delta=0.5 \delta_{0}$ respectively. The left panel is associated to the filament with $\omega=20^{\circ}$ and the right one is coresponding to the filament with $\omega=60^{\circ}$

\subsection{Summary}

In this chapter, we have explicitly evaluated the analytical expressions for the probability density function of the position of the end tip of the kinked filament and also the filaments with regular kinks. In this study, we have considered two situation; non-thermal elastic response of the filament to external force and the mechanical properties of the system consisting of the filament and a stiff flat wall. The non thermal elastic force constant of the filament depends on geometrical parameters like the kink angle, the graft angle, length of the segments, number of segments (for the filament with regular kinks) and the direction of the force. We have shown that the non thermal elastic force constant of the filament with regular kink in longitudinal direction linearly depends on $\mathrm{m}^{-1}$ while it depends on $\mathrm{m}^{-3}$ in transverse direction. In the system of wall and the filament, the entropic effects also play a role due to the thermal fluctuations therefore it has a non-linear behaviour. 



\section{Elasticity of a semiflexible filament with a discontinuous tension due to motor protein}

\subsection{Model description}

In this chapter, we start with the wormlike chain model for a semiflexible filament of contour length $L$ which is attached to a point or wall on one side and pulled by a constant force, $\mathbf{f}_{\mathbf{e x t}}$, on the other side. Its Hamiltonian is given by

$$
H_{W L C}=\frac{\kappa}{2} \int_{0}^{L}\left(\frac{d \mathbf{t}}{d s}\right)^{2}-\mathbf{f}_{\text {ext }} \int_{0}^{L} \frac{d \mathbf{t}}{d s}
$$

Here $\mathbf{t}(s)$ is the tangent vector at arclength $s, 0 \leq s \leq L$ and $\kappa$ denotes the bending stiffness. We will treat the above model in the weakly bending approximation 53 . (Which is explained in chapter.2), assuming that the persistence length $l_{p}=\kappa /\left(k_{B} T\right)$ is much larger than the length $L$ of the filament.

In the Monge parametrization [54, the tangent of a semiflexible filament is given by $t(s)=\frac{1}{\sqrt{1+a_{1}^{2}(s)+a_{2}^{2}(s)}}\left(1, a_{1}(s), a_{2}(s)\right)$. We assume that the filament is oriented along the x-direction, either due to the grafting on the left side and/or the pulling force $\mathbf{f}_{\text {ext }}=f_{\text {ext }} \mathbf{e}_{x}$. In the weakly bending limit the transverse components of the tangent vector $a_{1}(s)$ and $a_{2}(s)$ are small. We therefore approximate $t_{x}(s)=$ $1-\frac{1}{2}\left[a_{1}^{2}(s)+a_{2}^{2}(s)\right]$ and $\left(\frac{d t(s)}{d s}\right)^{2}=\dot{a}_{1}^{2}(s)+\dot{a}_{2}^{2}(s)$ where the dot denotes the derivative with respect to $s$. The Hamiltonan then reads

$$
H_{W B}=\sum_{i=1}^{2}\left[\int_{0}^{L}\left(\frac{\kappa}{2} \dot{a}_{i}^{2}(s)+\frac{f_{\text {ext }}}{2} a_{i}^{2}(s)\right) d s\right]
$$

Here, we are interested in the effects of a motor whose one end is grafted to a substrate while the other end is attached to the filament at arclength $L_{m}$. In the simplest model, the motor is just a spring of rest length $L_{0}$. and spring constant $k_{m}$

$$
H_{\text {spring }}=\frac{k_{m}}{2}\left(R_{1}-X_{0}-L_{0}\right)^{2}
$$



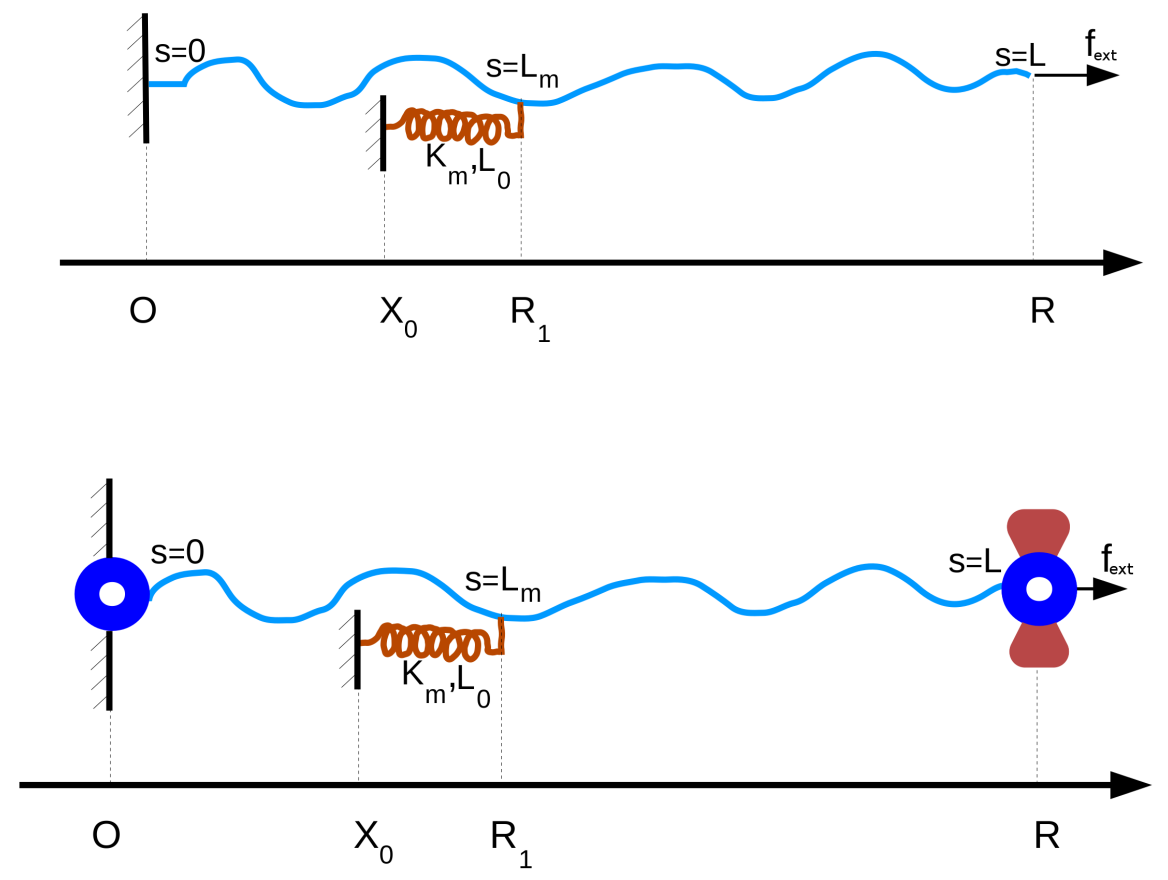

Figure 5.1: Schematic presentation of a filament attached to a spring with clamped-free and hinged-hinged boundary conditions at the ends. One end of the spring is fixed to the substrate. The spring pulls one side of the filament and pushes the other side. Therefore, the two sides of the filament are at different tension.

When the motor steps along the filament, its effective spring is compressed or extended beyond the rest length, resulting in a force, $f_{m}$ on the filament. Since the pulling force, $f_{\text {ext }}$, is fixed the presence of the motor will result in a compression or extension of the filament. In this paper, we compute the change in the forceextension relation of the filament due to the attached motor.

In the weakly bending approximation, we can represent the total Hamiltonian $H=H_{W B}+H_{\text {spring }}$ in the form of a filament with an arclength dependent tension:

$$
H=\sum_{i=1}^{2}\left[\int_{0}^{L}\left(\frac{\kappa}{2} \dot{a}_{i}^{2}(s)+\frac{f(s)}{2} a_{i}^{2}(s)\right) d s\right]
$$

where $f(s)$ is a piecewise constant function

$$
f(s)= \begin{cases}f_{\text {ext }}+f_{m} & 0<s<L_{m} \\ f_{\text {ext }} & L_{m}<s<L\end{cases}
$$


with $f_{m}=k_{m}\left(X_{0}-L_{m}+L_{0}\right)$.

Actually the assumtion of a harmonic spring for the motor is not needed as long as we use the weakly bending approximation. Consider a general interaction potential instead $V\left(R_{1}\right)$. In the weakly bending approximation, we take

$$
R_{1}-L_{m}=-\frac{1}{2} \sum_{i=1}^{2} \int_{o}^{L_{m}} a_{i}^{2}(s) d s
$$

to be small, and expand $V$ around $R_{1}=L_{m}$

$$
\begin{aligned}
V\left(R_{1}\right) & =V\left(L_{m}\right)+\frac{\partial V}{\partial R_{1}}\left(R_{1}-L_{m}\right) \\
& =V\left(L_{m}\right)+\frac{f_{m}}{2} \sum_{i=1}^{2} \int_{0}^{L_{m}} a_{i}^{2}(s) d s
\end{aligned}
$$

resulting in the same effective Hamiltonian (Eq 5.4), but now for a general interaction potential.

We want to compute the end-to-end distance of the filament $\langle R\rangle=\langle x(L)-x(0)\rangle$. To that end, we first calculate

$$
\left\langle R_{2}\right\rangle=\left(L-L_{m}\right)-\frac{1}{2} \sum_{i=1}^{2} \int_{L_{m}}^{L}\left\langle a_{i}^{2}(s)\right\rangle d s
$$

and similarly $\left\langle R_{1}\right\rangle$, where the thermal average $\langle\ldots\rangle$ is to be taken with the Hamiltonian of Eq.(5.4).

\subsection{Solution using Green function}

Using integration by parts for the first term in Eq. (5.4) we find:

$$
H_{W B}=\frac{1}{2} \sum_{i=1}^{2}\left[\int_{0}^{L} a_{i}(s) O(s) a_{i}(s) d s+B_{i}\right]
$$

where $B_{i}=\left.\frac{\kappa}{2} a_{i}(s) \dot{a}_{i}(s)\right|_{0} ^{L}$ depends on the boundary conditions and is a constant and $O(s)=-\kappa \frac{d^{2}}{d s^{2}}+f(s)$ is a differential operator. The corresponding Green function obeys the differential equation

$$
\beta\left(-\kappa \frac{d^{2}}{d s^{2}}+f(s)\right) G\left(s, s^{\prime}\right)=\delta\left(s-s^{\prime}\right)
$$

For a piecewise constant force $f(s)$ we can solve for the Green function in the two regions with constant force and then match the solutions at $s=L_{m}$ (see appendix). 
For the explicit calculation, we have to specify boundary conditions at both ends of the filament. We consider two cases: the clamped-free filament, shown in the upper part and the hinged-hinged filament, shown in the lower part of Fig.5.1.

\subsubsection{Clamped-free filament}

We require $\dot{a}_{i}(L)=0$ at the free end and $a_{i}(0)=0$ at the clamped end. For the Green function this implies

$$
\left.G_{c f}\left(s, s^{\prime}\right)\right|_{s=0}=\left.0 \quad \frac{\partial}{\partial s} G_{c f}\left(s, s^{\prime}\right)\right|_{s=L}=0
$$

The correlation function of the transverse components of the tangent vector can be obtained from the Green function as follows [55, 56]:

$$
\left\langle a_{i}(s) a_{i}\left(s^{\prime}\right)\right\rangle=G_{c f}\left(s, s^{\prime}\right)
$$

If no motor is attached, the force-extension relation reads [54]:

$$
\langle R\rangle_{W L C}=\left\langle R_{1}+R_{2}\right\rangle=L-\frac{L^{2}}{2 l_{p}}\left(\frac{\tanh \left(\tilde{f}_{\text {ext }}\right)}{\tilde{f}_{\text {ext }}}\right)
$$

The characteristic energy scale of the WLC is given by $\kappa / L$. Hence we have rescaled the externally applied pulling force with the bending force of the wormlike chain and introduced $\tilde{f}_{\text {ext }}=f_{\text {ext }} L^{2} / \kappa$. We get a linear relation for small forces

$$
f_{\text {ext }}=k_{\|}^{c f}(L)\left(L_{r}-\langle R\rangle\right)
$$

with rest length $L_{r}=L-L^{2} / 2 l_{p}$ and stiffness $k_{\|}^{c f}(L)=\frac{6 l_{p}^{2}}{\beta L^{4}}[54]$.

\subsubsection{Hinged-hinged filament}

In this case we require $\dot{a}_{i}(L)=0$ and $\dot{a}_{i}(0)=0$ implying for the Green function

$$
\left.\frac{\partial}{\partial s} G_{h h}\left(s, s^{\prime}\right)\right|_{s=0}=\left.0 \quad \frac{\partial}{\partial s} G_{h h}\left(s, s^{\prime}\right)\right|_{s=L}=0 .
$$

Fo a compressive external force $f_{\text {ext }}$, the filament is free to rotate at the grafted end. This can be prevented by requiring that the pulling point has to have the same height as the grafting point: $\int_{0}^{L} a_{i}(s)=0$. The correlation function of the components of the tangent vector is then given by [55, 56]:

$$
\left\langle a_{i}(s) a_{i}\left(s^{\prime}\right)\right\rangle=-\frac{\int_{0}^{L} G_{h h}\left(s, s_{1}\right) d s_{1} \int_{0}^{L} G_{h h}\left(s^{\prime}, s_{2}\right) d s_{2}}{\int_{0}^{L} \int_{0}^{L} G_{h h}\left(s_{1}, s_{2}\right) d s_{1} d s_{2}}
$$




$$
+G_{h h}\left(s, s^{\prime}\right)
$$

If no motor is attached to the filament, the force-extension is explicitly given by

$$
\langle R\rangle_{W L C}=L-\frac{L^{2}}{2 l_{p}}\left(\frac{\operatorname{coth}\left(\tilde{f}_{\text {ext }}\right)}{\tilde{f}_{\text {ext }}}-\frac{1}{\tilde{f}_{\text {ext }}^{2}}\right) .
$$

For small forces, the filament behaves like a spring

$$
f_{\text {ext }}=k_{\|}^{h h}(L)\left(L_{r}-\langle R\rangle\right)
$$

with rest length $L_{r}=L-L^{2} /\left(6 l_{p}\right)$ and a length dependent stiffness $k_{\|}^{h h}(L)=\frac{90\left(l_{p}\right)^{2}}{\beta L^{4}}$

\subsection{Analytical Results}

The explicit analytic solution for the Green function is given in the appendix. As a result we obtain analytic, albeit lengthy expressions for the force extension $\langle R\rangle=$ $\langle R\rangle\left(f_{\text {ext }}\right)$. To better understand these results, we plot the force-extension relations for hinged-hinged boundary conditions in Fig. 5.2. The effect of the motor force is more pronounced in the compressional regime, $f_{\text {ext }}<0$, because the filament is softer in response to compressions as compared to extensions, $f_{\text {ext }}>0$. The motor force can partially compensate the compression of the fiber by the external force, if its sign is opposite, i.e. it is pulling on the left part of the segment. Obviously, the left segment is then extended as compared to the case without motor (see Fig. 5.3 . upper part), but also the right segment is extended (see Fig 5.3 , lower part), even though the tension in the right part does not depend on $f_{m}$. The reason for this extension is the stronger alignment of the left end of the right part of the filament by the motor. The overall effect of a positive motor force is to substantially stiffen the filament in the compressional regime. The effects of course increase with increasing motor force. If the motor force is compressional the extension of the filament is correspondingly reduced as compared to the case without motor force.

In Fig. 5.4, we show the dependence of the filaments extension on the motor force, $f_{m}$, for several values of external pulling force, $f_{\text {ext }}$. If no external force is applied $\langle R\rangle=\langle R\rangle\left(f_{m}\right)$ looks qualitatively similar to $\langle R\rangle=\langle R\rangle\left(f_{\text {ext }}\right)$. The filament is most sensitive to the external pulling force in the range where the motor tends to compress the filament.

The effects of the motor are seen best in the differential tensile stiffness of the filament, which can be computed from the force-extension relation according to

$$
E^{-1}=\frac{\partial\langle R\rangle}{\partial f_{e x t}}
$$




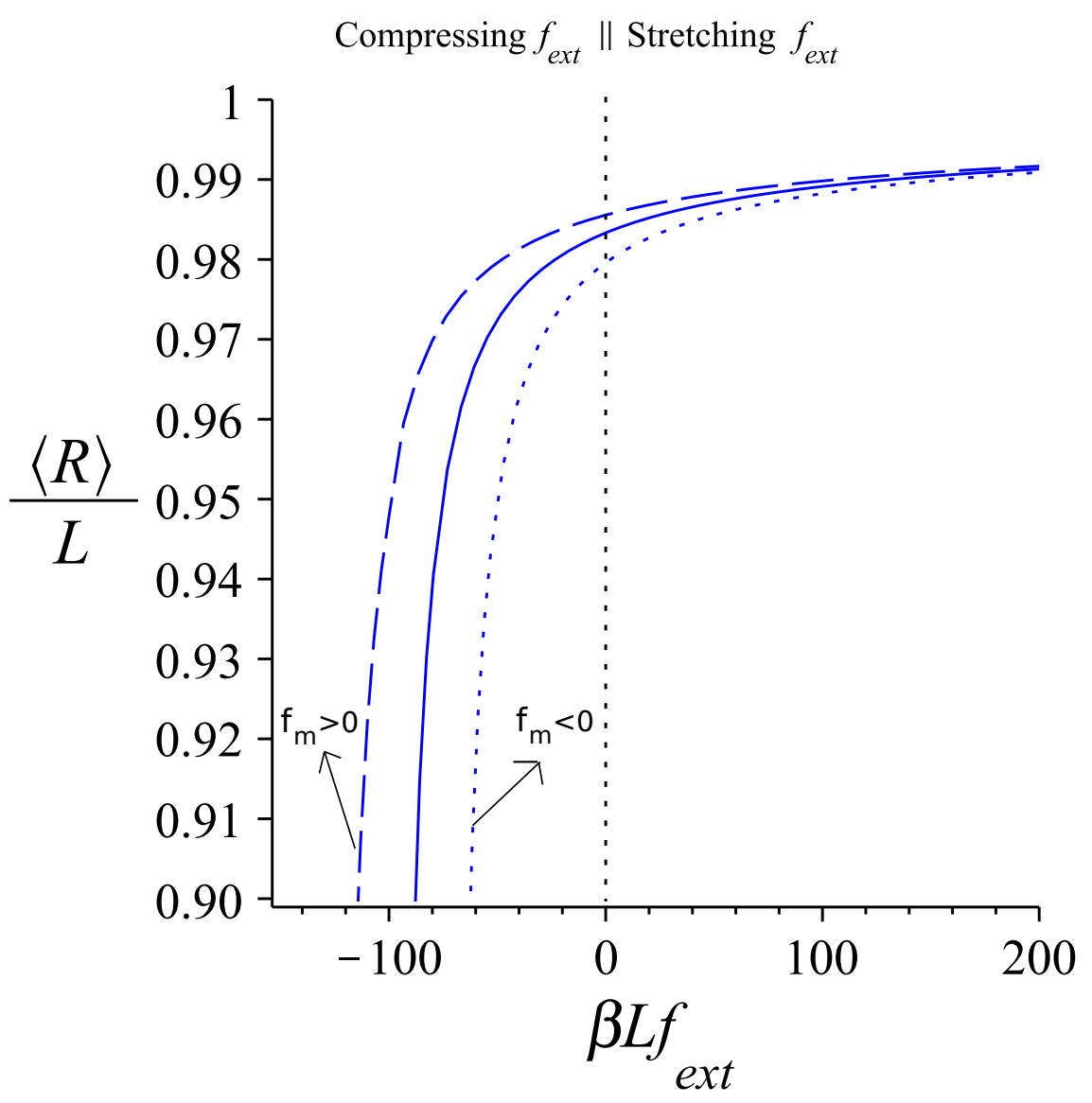

Figure 5.2: Extension of the filament, $\langle R\rangle$, as a function of the external force $f_{\text {ext }}$ which can be compressive or extensile; full line: no motor attached; dashed line $\beta L f_{m}=+50$; dotted line: $\beta L f_{m}=-50 ;$ (parameters: $L=1, l_{p}=10$, $L / L_{m}=2$; hinged-hinged filament)

In Fig. 5.5 we show the relative change in the differential stiffness of the filament caused by the spring. There is significant enhancement in stiffness when the spring force is extensile (dashed line), because the effective tension of the filament is increased. The stiffness is weakened for a compressive motor force (dots). In both cases do we observe stronger effects in the regime where the external force is compressive, implying that a filament under compression is strongly sensitive to a motor which is either pushing or pulling. 

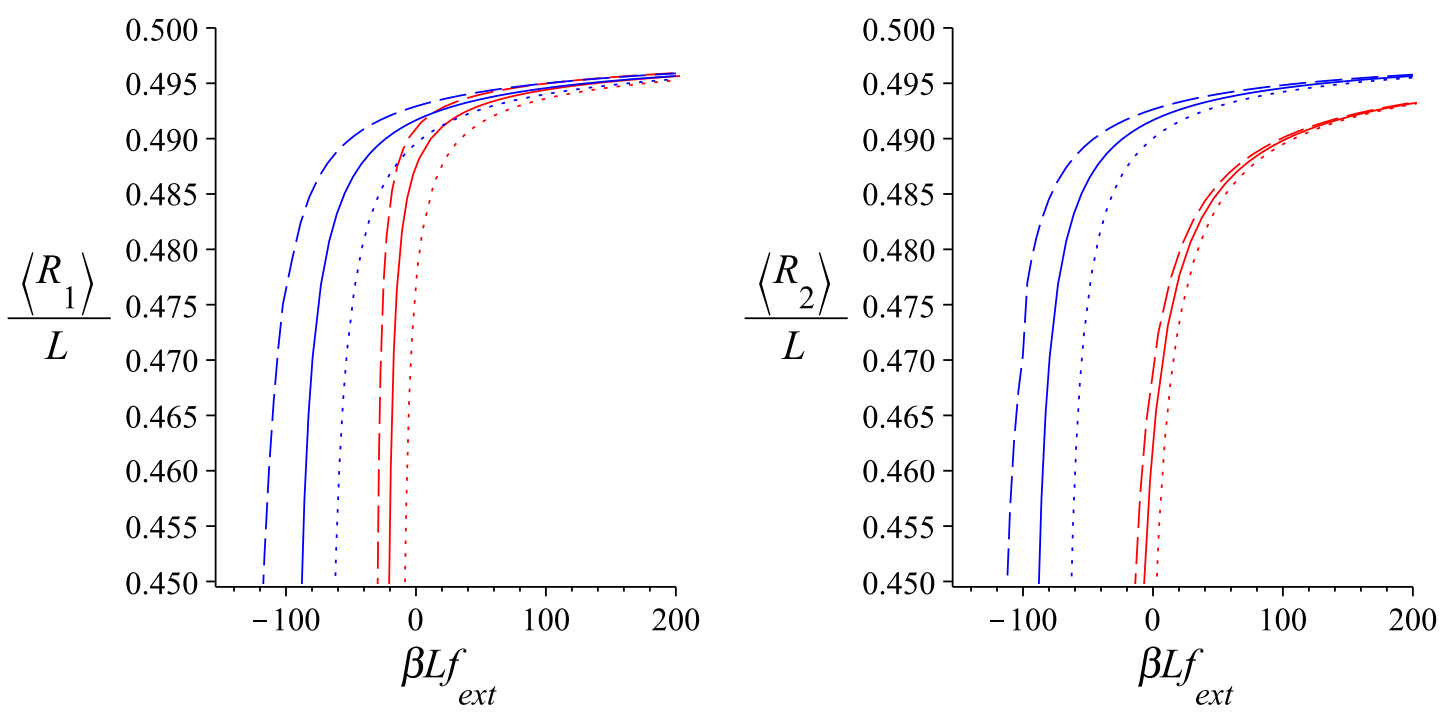

Figure 5.3: Extension of the two separate parts of the filament, $\left\langle R_{1}\right\rangle$ and $\left\langle R_{2}\right\rangle$, as a function of the external force, $f_{\text {ext }}$, for several values of the motor force; red lines: clamped-free filament; blue lines: hinged-hinged filament. (full lines: no motor attached; dashed lines $\beta L f_{m}=+50$; dotted lines: $\beta L f_{m}=-50$; parameters as in Fig 5.2

\subsubsection{Limit of small motor force $f_{m}$}

It is instructive to consider the limit of small motor force $f_{m} \ll \min \left\{f_{\text {ext }}, \frac{\kappa}{L^{2}}\right\}$. For the clamped-free case, we find

$$
\langle R\rangle-\langle R\rangle_{f_{m}=0}=\frac{f_{m}}{k\left(f_{\text {ext }}\right)} .
$$

In this limit, the motor-filament system can be represented as a linear elastic element with an effective force constant $k$ that depends on the external pulling force and the point of attachment of the motor. The explicit expression for the force constant is given in the Appendix, and a similar expression can be calculated for the hingedhinged case. In Fig. 5.6, we show the force constant $k$ of the motor as a function of the external force $f_{\text {ext }}$. The force constant decreases as we compress the filament and it increases as we increase the stretching force. This change of the motor force constant $k$ is an essential feature of the elasticity of the semiflexible filament which is missing in studies using linear elasticity for the filament. In Fig. 5.7, we compare the exact force-extension relation to the linearised one. As can be seen in the figure, the linear approximation works better for higher values of the external force $f_{\text {ext }}$.

In the limit of large external forces, $f_{\text {ext }} \gg \max \left\{f_{m}, \frac{\kappa}{L_{m}{ }^{2}}\right\}$, and large filament 


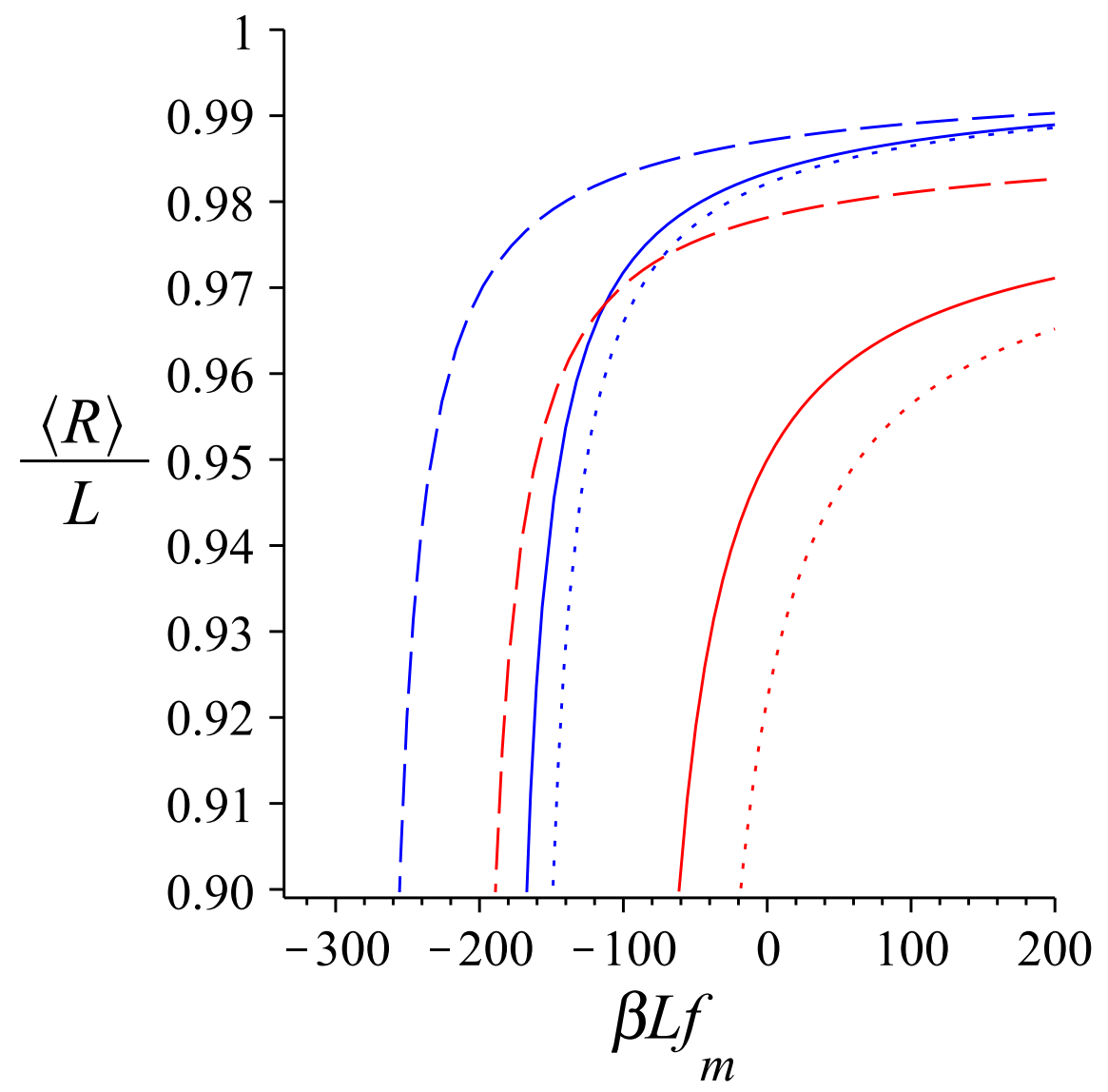

Figure 5.4: Extension of the filament, $\langle R\rangle$, as a function of the motor force $f_{m}$ for several values of the external force; red lines: clamped-free filament, blue lines: hingedhinged filament. (full lines: no motor attached; dashed lines $\beta L f_{\text {ext }}=+50$; dotted lines: $\beta L f_{\text {ext }}=-10$; parameters as in Fig. 5.2 .

length, $\min \left\{L, L_{m}\right\} \gg f_{\text {ext }} /\left(k_{B} T\right)$, Eq. 5.19 reduces to the following relation, irrespective of boundary conditions:

$$
\frac{\langle R\rangle}{L}=1-\frac{1}{2} \frac{k_{B} T}{\sqrt{\kappa f_{\text {ext }}}}+\frac{L_{m}}{4 L} \frac{k_{B} T f_{m}}{\sqrt{\kappa} f_{\text {ext }}{ }^{\frac{3}{2}}} .
$$

Notice that this equation holds in the thermodynamic limit and it is scale invariant: if we multiply all lengths $\left(\langle R\rangle, L_{m}, L\right)$ by the same factor, it does not change. The effect of the motor force is subdominant, as it scales with $\sim 1 / f_{\text {ext }}^{3 / 2}$ compared to $\sim 1 / \sqrt{f_{\text {ext }}}$ for the Marko-Siggia case, but it is noteworthy that it persists in the thermodynamic limit and is not just a finite-size effect.

In the limit of small external forces, $f_{\text {ext }} \ll \kappa / L^{2}$, we obtain a linear response to 


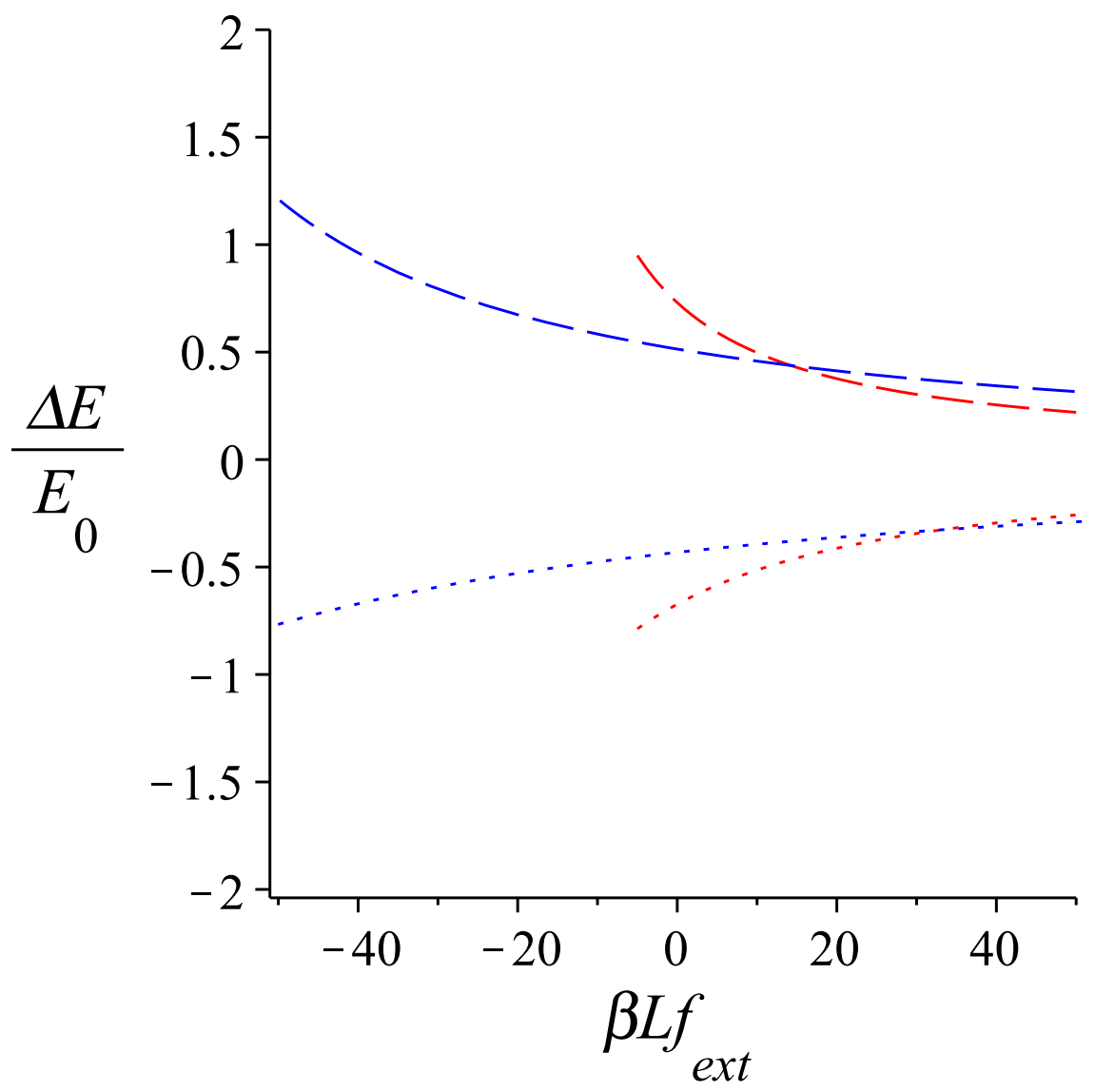

Figure 5.5: Change in the differential stiffness relative to the case without a spring as a function of the external force; red lines: clamped-free filament, blue lines: hinged-hinged filamen; (dashed lines $\beta L f_{m}=+50$; dotted lines: $\beta L f_{m}=-50$; parameters as in Fig 5.2 .

both the motor and the external force, which in the case of clamped-free boundary conditions reads:

$$
\frac{\langle R\rangle_{c f}}{L}=1-\frac{L}{2 l_{p}}+\frac{2 L_{m}^{3} L-L_{m}^{4}}{6 L^{3} l_{p}} \tilde{f}_{m}+\frac{L}{6 l_{p}} \tilde{f}_{\text {ext }}
$$

\subsubsection{Limit of large motor force $f_{m}$}

In the limit of large motor force, $f_{m} \gg \max \left\{f_{\text {ext }}, \frac{\kappa}{L_{m}{ }^{2}}\right\}$, we expect the left part of the filament to display the asymptotic (Marko-Siggia) force extension for large $f_{m}$ 


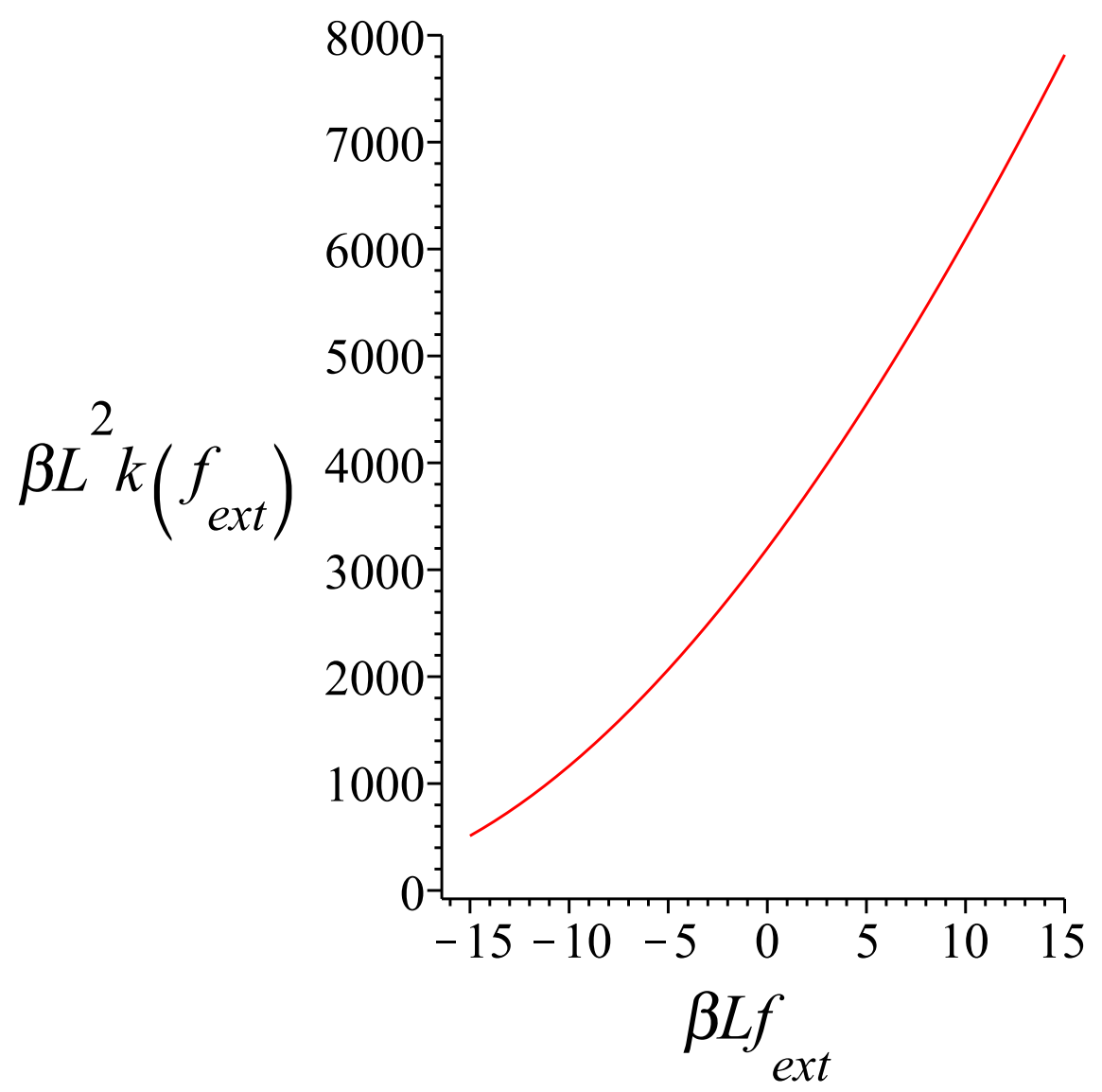

Figure 5.6: Effective stiffness, $k\left(f_{\text {ext }}\right)$, of the filament with respect to the motor force as a function of external force $f_{\text {ext }}$. The range of the external force is chosen in a way that the filament is well approximated as weakly bending $\left(\frac{R}{L}>0.9\right)$; parameters as in Fig 5.2 .

and indeed it does:

$$
\left\langle R_{1}\right\rangle_{c f}=L_{m}-\frac{L_{m}}{2 l_{p}} \sqrt{\frac{\kappa}{f_{m}}}
$$

However the extension of the right part of the filament is not just given by the expression for a wormlike chain under tension $f_{\text {ext }}$ but shows a correction of $\mathcal{O}\left(\frac{1}{\sqrt{f_{m}}}\right)$

$$
\left\langle R_{2}\right\rangle_{c f}=\left\langle R_{2}\right\rangle_{W L C}-\alpha\left(f_{e x t}\right) \sqrt{\frac{\kappa}{f_{m}}} .
$$

The strength of the effect depends on the external pulling force (see Fig. 5.8) and is strongest for weak pulling force. Explicit forms of $\left\langle R_{2}\right\rangle_{W L C}$ and $\alpha\left(f_{\text {ext }}\right)$ are given in the Appendix. 


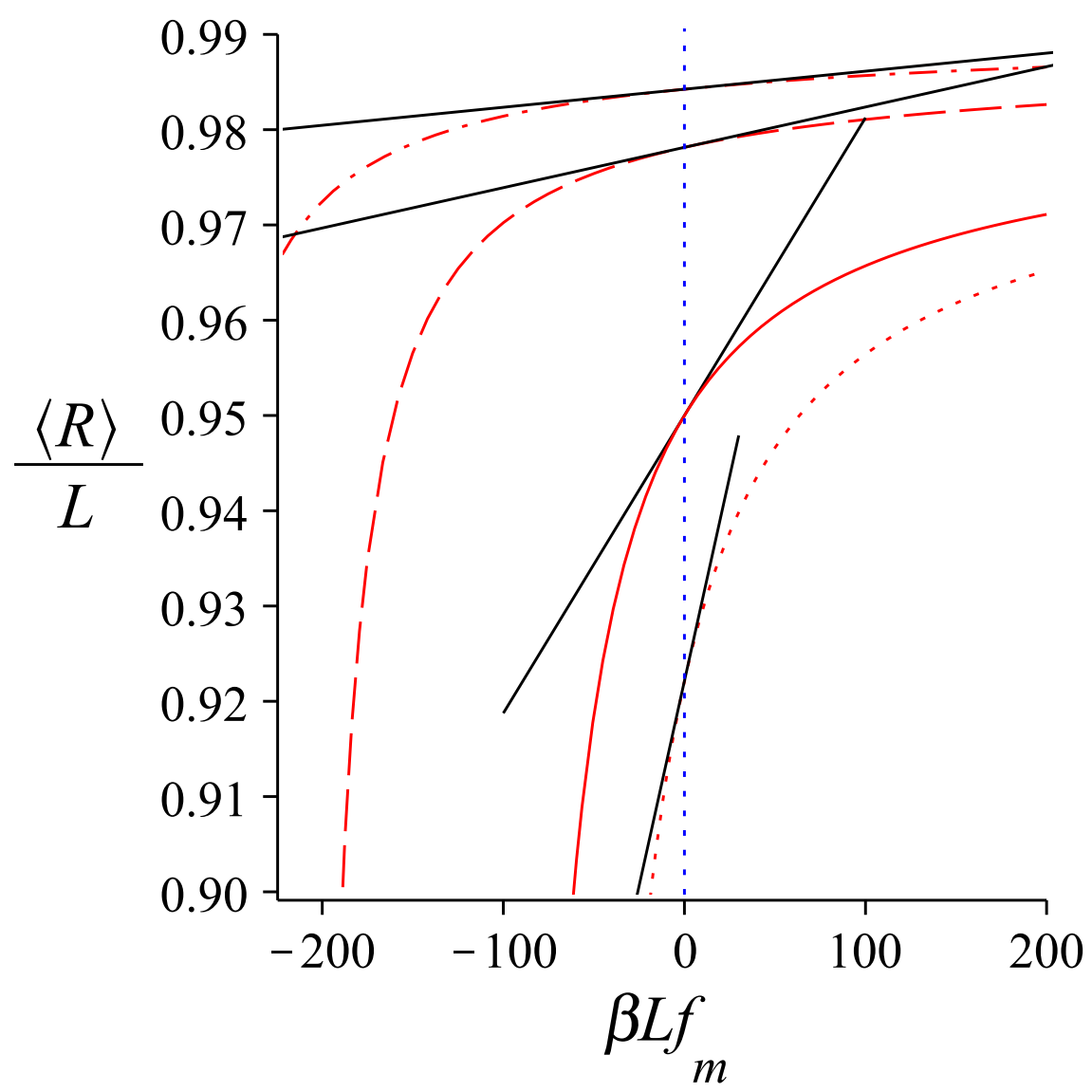

Figure 5.7: Comparison of the complete solution for the end to end distance $\langle R\rangle / L$ and the one linearized around $f_{m}=0$; full lines: $\beta L f_{\text {ext }}=0$; dashed lines $\beta L f_{\text {ext }}=50$; dashed-dotted lines: $\beta L f_{\text {ext }}=100$; dotted line: $\beta L f_{\text {ext }}=-10$ (parameters as in Fig 5.2 .

In the limit of small external force, $f_{e x t} \ll \kappa / L^{2} \ll f_{m}$ and $\kappa / L_{m}^{2} \ll f_{m}$, we obtain a linear force-extension relation:

$$
\begin{aligned}
\frac{\langle R\rangle_{c f}}{L} & =1-\frac{L}{2 l_{p}}+\frac{L_{m}\left(6 L-3 L_{m}\right)}{6 l_{p} L}+\frac{\left(L-L_{m}\right)^{4}}{6 L^{3} l_{p}} \tilde{f}_{e x t} \\
& -\frac{\left(6 L-3 L_{m}\right) L_{m}}{6 l_{p} L} \frac{1}{\tilde{f}_{m}^{\frac{1}{2}}} .
\end{aligned}
$$

\subsubsection{Limit of large force $f_{e x t}$ and $f_{m}=-f_{\text {ext }}+\epsilon$}

Next, we consider the limit of a large external pulling force, which is almost compensated by the motor in the left part of the filament. In other words, we put 


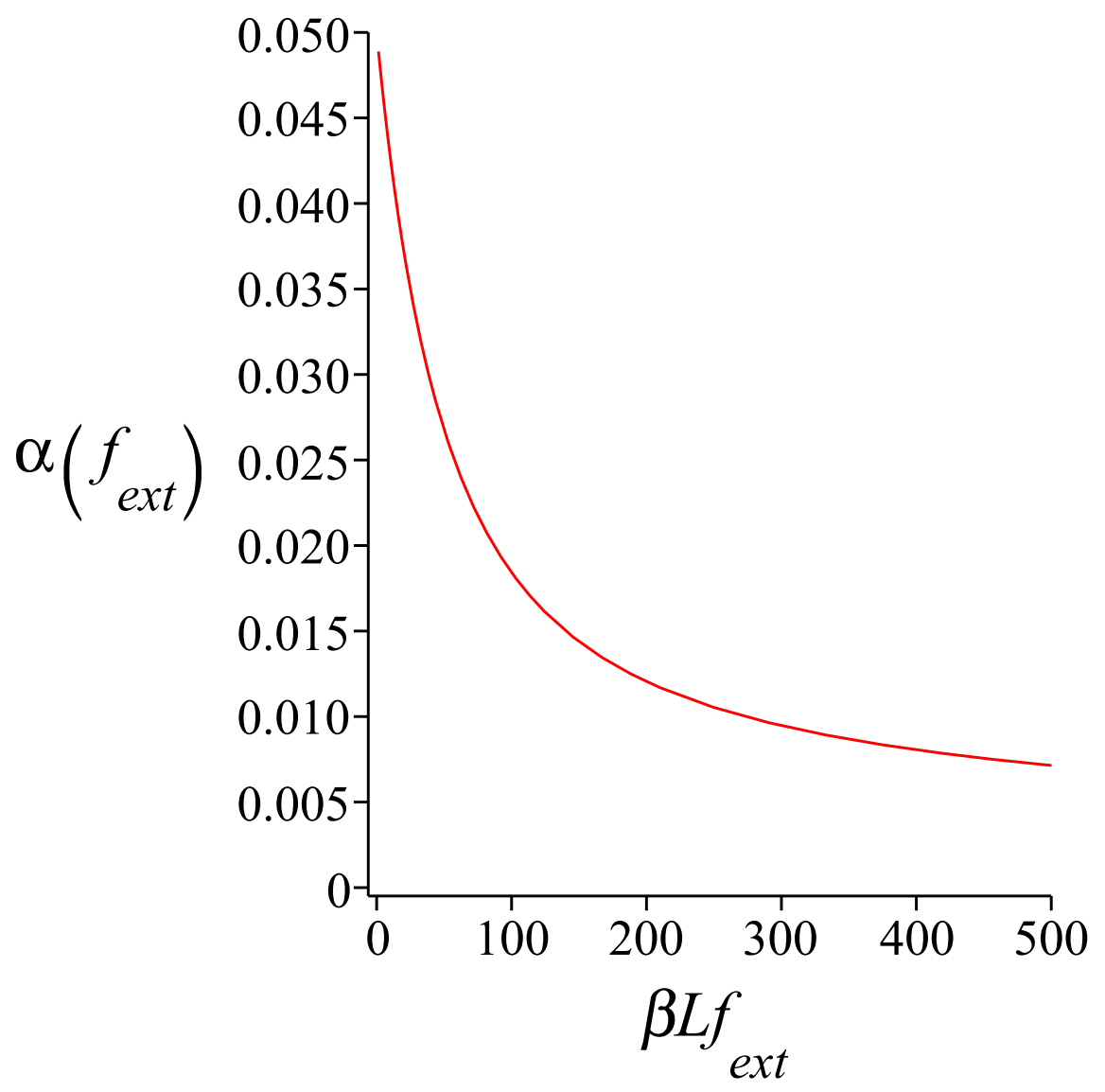

Figure 5.8: Coefficient of the asymptotic expansion, $\alpha\left(f_{\text {ext }}\right)$, as function of external force $f_{\text {ext }}$; (parameters as in Fig 5.2 .

$f_{m}=-f_{\text {ext }}+\epsilon$ and consider the case with $f_{\text {ext }} \gg \frac{\kappa}{L_{m}^{2}}$ and $\epsilon \ll \frac{\kappa}{L^{2}}$. In this limit, the right part of the filament is asymptotically extended

$$
\left\langle R_{2}\right\rangle_{c f}=L-L_{m}-\left(\frac{L-L_{m}}{2 l_{p}}\right)\left(\frac{\kappa}{f_{\text {ext }}}\right)^{1 / 2} .
$$

The total extension in this limit is given by

$$
\langle R\rangle_{c f}-L=\frac{L_{m}^{2}}{6 l_{p}}-\left(\frac{L}{6 l_{p}}\right) \frac{\left(3 L-L_{m}\right)}{\tilde{f}_{e x t}^{\frac{1}{2}}}+\left(\frac{L_{m}^{4}}{90 l_{p} L^{2}}\right) \tilde{\epsilon} .
$$

The first term accounts for the reduction in length due to thermal fluctuations in the left part only and hence $\propto L_{m}^{2}$. The tension in the left part is just $\epsilon \ll 1$ which accounts for the last term. However the pulling force $f_{\text {ext }}$ affects the orientation of the tangent at $L_{m}$ and hence also the extension of the left part of the filament so 
the dominant term for strong pulling force is not just determined by the right part of the filament.

\subsection{Relation to single-motor experiments}

Our results can be tested experimentally using optical tweezers. Beads attached to the two ends of the biomolecule which acts as track (e.g., F-actin) are trapped with optical tweezers. The motor (e.g., myosin-V) is attached with one end to a fixed bead and with the other end to the filament. Since the two end beads are free to rotate, this arrangement corresponds to the case of hinged-hinged boundary conditions. This experimental set-up has already been used in many single-molecule mechanical transducers [30]. The "three-bead" technique was pioneered by Finer et al. [57]. The main idea is to measure the variance of the end-beads' position which is related to the stiffness of the actomyosin cross-bridge complex within linear elasticity [58, 59]. Conformational changes in the motor induce changes in the effective stiffness of the bridge which is measured experimentally. In our model, the conformational change in myosin changes the motor force $f_{m}$. This can be due to a change in the position of attachment of the myosin head on the actin filament, $L_{m}$, or a change in the effective spring constant, $k_{m}$, or both.

Our results have been obtained in the fixed force ensemble, where the tension on the filament is determined sharply and this results in a fluctuating extension, whose average we have calculated. The positions of the motor bead, which determines $X_{0}$ in our model, and of the left filament end are held fixed. This can be done by using a very stiff optical trap. As shown by Gerland et al. in [60], a polymer held between two optical traps is represented by the mixed ensemble, where both the tension and the extension fluctuate. This mixed ensemble interpolates between the fixed extension ensemble (corresponding to the limit of very stiff traps) and fixed force ensemble (corresponding to the limit of very soft traps). Therefore, our general results for hinged-hinged boundary conditions can be tested with a set up involving a very soft optical trap for the right end of the filament. We should point out, that the force-extension relation given by Eq. 5.20 holds in the thermodynamic limit and as such is ensemble independent (fixed force or fixed extension). In addition, the linear response results are ensemble independent.

In real systems, the spring will act not only in the longitudinal direction but also in the transverse direction. The effect of a transverse spring of zero rest length in the force-extension relation of a weakly bending wormlike chain has been calculated in [61]. For a spring of finite rest length which is almost parallel to the longitudinal direction of the filament, the transverse effect is of higher order and can be neglected. For a spring of zero rest length but with $f_{m} \neq 0$, we can simply add the following 
contribution to the right-hand side of Eq. 5.20 .

$$
\frac{\Delta\langle R\rangle}{L}=\frac{k_{m} k_{B} T}{4 f_{\text {ext }}^{2}}\left(1+\frac{k_{m} L}{2 f_{\text {ext }}}\right)^{-1},
$$

which holds in the strong stretching limit, $f \gg \kappa / L_{m}^{2}$. For a soft spring, $k_{m} L \ll$ $f_{\text {ext }}$, this contribution falls off as $\sim f_{\text {ext }}^{-2}$, which is subdominant to the longitudinal contribution which falls off as $\sim f_{\text {ext }}^{-3 / 2}$.

\subsection{Summary}

In this chapter, we have studied mechanical properties of the filament with force discontinuity caused by a motor protein or a cross-linker having analytical calculations. The stiffness of the filament can be seen from two different response; The response to the motor force or the response to the external force. We have shown that stretching motor force stiffens the filament (in response to external force) while the compressive one softens it (in response to external force). This effect is more pronounced when the filament experiences a compressive external force. The stiffness of the filament in response to motor force changes as the external force changes. It is softer for compressive external force while the stretching external force makes it stiffer. We have also presented some analytical expressions for the force-extension relation of the filament which are valid in the limiting cases. 


\section{Conclusion and summary}

In this thesis, we have studied biopolymers in the weakly bending regime and the resulting basic structural element of the networks. In Chapter 3, we have shown that a single branched actin filaments with intermediate branch point positions and tilt angles exert larger forces and are stiffer than two unbranched actin filaments. The stiffness of a whole network of branched filaments is largest in comparison to the network of single filaments, if the orientation distribution is sharply peaked around $\pm \gamma / 2= \pm 35^{\circ}$. For the orientation distributions of lamellipodia, the stiffness of branched and unbranched networks scales approximately the same with a variety of parameters (Fig. 3.6), suggesting that the effect of branching on network stiffness can be accounted for by rescaling the filament number of an unbranched network. These results are in agreement with the elastic properties of lamellipodia found in force-velocity measurements with fish keratocytes [13, 11, 12].

In Chapter 4, we have given the analytical expressions for the probability density of the position of the end tip of a filament with one kink and also for the filament with regular kinks. Having the probability density, we have studied the elasticity of the structures. For the filament with regular kinks, we have shown that the elastic force constant in longitudinal direction is proportional to the inverse of the number of segments $m$ while it is proportional to the inverse of the cube of the number of segments $m^{-3}$ in the transverse direction.

In Chapter 5, We have analysed the force-extension of a wormlike chain whose one end is fixed, while the other end is pulled or pushed by an external force. In addition, the filament is attached to a spring which may represent a cross-link or a motor arrested at its stall force. Irrespective of boundary conditions, the effects of the spring are stronger in the compressive regime as compared to the stretching regime. Depending on the relative sign of the pulling force and the spring force, the latter can substantially stiffen or weaken the filament. When the motor force is small, its effects can be represented by an effective spring constant which strongly depends on the prestress of the fiber, i.e. the external force. When the motor force is large, it gives rise to a $1 / \sqrt{f_{\text {ext }}}$ dependence which is well known from the work of Marko and Siggia [53]. The dependence of the force extension curve of the filament on the motor force allows to deduce the latter from measurements of the force-extension relation. In fact the so-called three bead geometry has already been used to determine the stiffness of the actomyosin cross-bridge [59].

An interesting direction for future work is the study of two or more parallel-aligned filaments with non-local spring-like cross-linkers in the direction of alignment. The 
case of local cross-links has been investigated in [62, 63, 61]. A simplified model of two filaments with a non-local spring has been studied in [64]. 
Appendices 



\section{A Mechanical properties of branched actin filaments}

\section{A.1 Tilted filament}

The probability distribution of the position $x$ of the endpoint follows by integration (see Eq. 3.7)

$$
P_{t}\left(x \mid x_{0}\right) \propto \exp \left(-\frac{\left(x-x_{0}-L \cos (\omega)\right)^{2}}{\sigma_{t 0}^{2}}\right)
$$

where

$$
\sigma_{t 0}^{2}=\frac{4 L^{3} \sin (\omega)^{2}}{3 l_{p}} .
$$

Representing the stiffness of the graft by a spring and averaging over all grafting points, $x_{0}$, yields:

$$
P_{t}(x)=\frac{1}{N_{t}} \exp \left(-\frac{(x-L \cos (\omega))^{2}}{\sigma_{t}^{2}}\right) .
$$

Here $N_{u}$ accounts for the proper normalization and

$$
\sigma_{t}^{2}=\sigma_{t 0}^{2}+\frac{2}{K}
$$

The entropic force on the wall is then explicitly given by:

$$
f_{t}(\delta)=\frac{2 k_{B} T}{\left(\sqrt{\pi} \sigma_{t}\right)} \frac{\exp \left(-\frac{(\delta-L \cos (\omega))^{2}}{\sigma_{t}^{2}}\right)}{\operatorname{erfc}\left(\frac{L \cos (\omega)-\delta}{\sigma_{t}}\right)} .
$$

\section{A.2 Branched filament}

Starting from Eq. 3.10 and averaging over graft point positions $x_{0}$, we obtain

$$
P_{b}\left(x_{2}, x_{3}\right)=\left(\frac{(\operatorname{det}(\mathrm{M}))^{\frac{1}{2}}}{\pi}\right) \exp \left(\eta_{i} M_{i j} \eta_{j}\right)
$$


where

$$
\eta=\left(\begin{array}{c}
x_{2}-L_{1} \cos (\omega)-L_{b} \cos (\omega-\gamma) \\
x_{3}-\left(L_{1}+L_{3}\right) \cos (\omega)
\end{array}\right)
$$

and $M$ is a $2 \times 2$-Matrix with components

$$
\begin{aligned}
& M_{11}=-\frac{l_{p}}{C}\left[K\left(L_{1}+L_{3}\right)^{3} \sin (\omega)^{2}+\frac{3}{2} l_{p}\right] \\
& M_{12}=M_{21}=\quad \frac{l_{p}}{C}\left[K L_{1}^{2}\left(L_{1}+\frac{3}{2} L_{3}\right) \sin (\omega)^{2}+\frac{3}{2} l_{p}+\right. \\
& \left.\frac{3}{2} K L_{1} L_{b}\left(L_{1}+2 L_{3}\right) \sin (\omega) \sin (\omega-\gamma)\right] \\
& M_{22}=\frac{-l_{p}}{C}\left[K L_{b}^{2}\left(3 L_{1}+L_{b}\right) \sin (\omega-\gamma)^{2}+\frac{3}{2} l_{p}+\right. \\
& \left.3 K L_{1}^{2} L_{b} \sin (\omega) \sin (\omega-\gamma)+K L_{1}^{3} \sin (\omega)^{2}\right] \text {, }
\end{aligned}
$$

and

$$
\begin{gathered}
C=K \sin (\omega)^{2}\left[\left(L_{1}+\frac{4}{3} L_{3}\right) L_{1}^{3} L_{3}^{2} \sin (\omega)^{2}\right. \\
\left.-2\left(L_{1}^{2}-2 L_{3}^{2}\right) L_{1}^{2} L_{b} L_{3} \sin (\omega) \sin (\omega-\gamma)\right] \\
+L_{b}^{2} K \sin (\omega)^{2} \sin (\omega-\gamma)^{2}\left[L_{1}^{4}+\frac{4}{3}\left(L_{1}^{3} L_{b}+L_{b} L_{3}^{3}\right)\right. \\
\left.+4 L_{1}^{2} L_{b} L_{3}+4 L_{1} L_{3}^{2}\left(L_{b}+L_{3}\right)\right] \\
+6 l_{p}\left[\left(L_{1}+\frac{1}{3} L_{3}\right) L_{3}^{2} \sin (\omega)^{2}-2 L_{1} L_{b} L_{3} \sin (\omega) \sin (\omega-\gamma)\right. \\
\left.+\left(L_{1}+\frac{1}{3} L_{b}\right) L_{b}^{2} \sin (\omega-\gamma)^{2}\right]
\end{gathered}
$$

\section{A.3 Parameter values}

The independent parameters of the model are the F-actin persistence length $l_{p}$, the equilibrium distance of the filament tips from the graft plane $\delta_{0}$, the stiffness $K$ of the graft, and the branching angle $\gamma$ which is fixed by the Arp2/3 complex at about $70^{\circ}$ [65, 66, 67]. The branch point position is uniformly distributed and the tilt angle $\omega$ according to the above distributions. The tilt angle and $\delta_{0}$ fix the mother filament contour length as $L=\delta_{0} / \cos (\omega)$.

We explained in the Discussion, that a region juxtaposed to the leading edge membrane with a width of $1.0-1.5 \mu \mathrm{m}$ is similar to an experimental realization of the network configuration in Fig. 3.1, since it is weakly cross-linked. Values for $\delta_{0}$ suggested by these observations are in the range 1.0-1.5 $\mu \mathrm{m}$. Branching will have an effect on network properties in a configuration like shown in Fig. 3.1, if most filaments are branched, i.e., if $\delta_{0}$ is larger than the average branch distance.

The average branch distance in steadily moving cells has been a matter of debate 
in recent years. Svitkina et al. concluded 20-50 nm from early electron micrographs of lamellipodia from Xenopus keratocytes and fibroblasts [15], and 50-200 nm from another study in fibroblasts [68]. Later measurements substantially increased that value. The average branch distance has been determined to be about $800 \mathrm{~nm}$ for B16 melanoma cells and fish keratocytes in ref. [47]. Other studies provide number densities of branch points per lamellipodium area for 3T3 fibroblasts. Calculating the average branch distance from that branch point density implies assumptions on the F-actin concentration. The number of filaments per micrometer lamellipodium width in a distance of $0.1-1 \mu \mathrm{m}$ from the leading edge is 170-190 [41. Taking into account that filament orientation is approximately uniformly distributed between $0^{\circ}$ and $60^{\circ}$ [39], this density means 1.16(170-190) $\mu$ m filament contour length per $\mu \mathrm{m}^{2}$ lamellipodium area. The factor 1.16 arises from averaging over all tilt angles of the filaments. Yang and Svitkina measured 277 branch points $/ \mu \mathrm{m}^{2}$ [67] in the same sample, i.e. an average branch point distance in terms of contour length between $700 \mathrm{~nm}$ and $800 \mathrm{~nm}$. Small et al. measured less than 225 branch points $/ \mu^{2}{ }^{2}$ [42], i.e. an average distance of more than $860 \mathrm{~nm}$.

The in vivo persistence length is not known. We can only conclude a reasonable range from in vitro measurements. Results from fluctuation analysis and measurements of network elastic properties yield values of the in vitro persistence length of 15-18 $\mu \mathrm{m}$ [49, 69, 9, 70]. The filaments in these experiments were stabilized with phaloidin which most likely increases the value of $l_{p}$. Filaments labeled with rhodamine but not stabilized with phaloidin exhibited values of $l_{p}$ between $9 \mu \mathrm{m}$ and $13.5 \mu \mathrm{m}$ [70]. The in vivo persistence length might be even shorter, since cofilin can substantially reduce it even down to $2.2 \mu \mathrm{m}$ [71, 72]. 



\section{B Mechanical properties of Filament with kinks}

\section{B.1 Kinked filament with even number of arms}

Here we want to use mathematical induction to prove a guess for probability density function of the regularly kinked filament with even number of arms. The following relation should be satisfied by the probability density function of the regularly kinked filament with even number of arms $m=2 n$ :

$$
\begin{aligned}
P_{e}\left(x_{2 n+2}, y_{2 n+2}, \theta_{2 n+2}\right)= & \iiint \iiint d x_{2 n} d y_{2 n} d \theta_{2 n} d x_{2 n+1} d y_{2 n+1} d \theta_{2 n+1} P_{e}\left(x_{2 n}, y_{2 n}, \theta_{2 n}\right) \\
& \times G_{L}\left(x_{2 n+1}, y_{2 n+1}, \theta_{2 n+1} \mid x_{2 n}, y_{2 n}, \theta_{2 n}+2 \omega\right) \\
& \times G_{L}\left(x_{2 n+2}, y_{2 n+2}, \theta_{2 n+2} \mid x_{2 n+1}, y_{2 n+1}, \theta_{2 n+1}-2 \omega\right)
\end{aligned}
$$

The probability density function of regularly kinked filament with even number of arms satisfying the aforementioned relation for mathematical induction is:

$$
P_{e}\left(x_{2 n}, y_{2 n}, \theta_{2 n}\right) \propto \exp \left(A \theta_{2 n}^{2}+B \theta_{2 n}+C\right)
$$

Where

$$
\begin{gathered}
A=\frac{-7 l_{p}}{4(2 n) L} \\
B=\frac{3 l_{p}}{(2 n)^{2} L^{2}} \frac{(2 n) \eta_{x} \cos (\omega)+\eta_{y} \sin (\omega)}{\sin (\omega) \cos (\omega)} \\
C=\frac{-3 l_{p}}{(2 n)^{3} L^{3}} \frac{(2 n)^{2} \eta_{x}^{2} \cos (\omega)^{2}+\eta_{y}^{2} \sin (\omega)^{2}}{\sin (\omega)^{2} \cos (\omega)^{2}}
\end{gathered}
$$

Also, we have defined $\eta_{x}=x_{2 n}-(2 n) L \cos (\omega)$ and $\eta_{y}=y_{2 n}-\left(\frac{1-(-1)^{2 n}}{2}\right) L \sin (\omega)=$ $y_{n}$. Now one can calculate $P_{e}\left(x_{2 n}\right)$ which is the following expression:

$$
P_{e}\left(x_{2 n}\right)=\iint d y_{2 n} d \theta_{2 n} P_{e}\left(x_{2 n}, y_{2 n}, \theta_{2 n}\right) \propto \exp \left(-\frac{3}{4} \frac{l_{p} \eta_{x}^{2}}{(2 n) L^{3} \sin (\omega)^{2}}\right)
$$


Also we can calculate $P_{e}\left(y_{2 n}\right)$ :

$$
P_{e}\left(y_{2 n}\right)=\iint d x_{2 n} d \theta_{2 n} P_{e}\left(x_{2 n}, y_{2 n}, \theta_{2 n}\right) \propto \exp \left(-\frac{3}{4} \frac{l_{p} \eta_{y}^{2}}{(2 n)^{3} L^{3} \cos (\omega)^{2}}\right)
$$

\section{B.2 Kinked filament with odd number of arms}

Having the probability density function of the regularly kinked filament with even number of arms, we can calculate $P_{o}\left(x_{2 n+1}\right)$ with the following integration:

$$
\begin{aligned}
P_{o}\left(x_{2 n+1}\right)= & \iiint \iint d x_{2 n} d y_{2 n} d \theta_{2 n} d y_{2 n+1} d \theta_{2 n+1} P_{e}\left(x_{2 n}, y_{2 n}, \theta_{2 n}\right) \\
& \times G_{L}\left(x_{2 n+1}, y_{2 n+1}, \theta_{2 n+1} \mid x_{2 n}, y_{2 n}, \theta_{2 n}+2 \omega\right)
\end{aligned}
$$

The result is:

$$
P_{o}\left(x_{2 n+1}\right) \propto \exp \left(-\frac{3}{4} \frac{l_{p} \eta_{x}^{2}}{(2 n+1) L^{3} \sin (\omega)^{2}}\right)
$$

We can also calculate $P_{o}\left(y_{2 n+1}\right)$ with the following integration:

$$
\begin{aligned}
P_{o}\left(y_{2 n+1}\right)= & \iiint \iint d x_{2 n} d y_{2 n} d \theta_{2 n} d x_{2 n+1} d \theta_{2 n+1} P_{e}\left(x_{2 n}, y_{2 n}, \theta_{2 n}\right) \\
& \times G_{L}\left(x_{2 n+1}, y_{2 n+1}, \theta_{2 n+1} \mid x_{2 n}, y_{2 n}, \theta_{2 n}+2 \omega\right)
\end{aligned}
$$

The result is:

$$
P_{o}\left(y_{2 n+1}\right) \propto \exp \left(-\frac{3}{4} \frac{l_{p} \eta_{y}^{2}}{(2 n+1)^{3} L^{3} \cos (\omega)^{2}}\right)
$$

Similar to the previous subsection, we have defined $\eta_{x}=x_{2 n+1}-(2 n+1) L \cos (\omega)$ and $\eta_{y}=y_{2 n+1}-\left(\frac{1-(-1)^{2 n+1}}{2}\right) L \sin (\omega)=y_{n}-L \sin (\omega)$.

\section{B.3 The general case}

Having the probability density function for the regularly kinked filament with both even and odd number of arms, we write the following expression for the probability density function of filament with $m$ number of arms ( $m$ is any integer number):

$$
P\left(x_{m}\right) \propto \exp \left(-\frac{3}{4} \frac{l_{p} \eta_{x}^{2}}{m L^{3} \sin (\omega)^{2}}\right)
$$

and similarly for $P\left(y_{m}\right)$ :

$$
P\left(y_{m}\right) \propto \exp \left(-\frac{3}{4} \frac{l_{p} \eta_{y}^{2}}{m^{3} L^{3} \cos (\omega)^{2}}\right)
$$


Where we have defined $\eta_{x}=x_{m}-m L \cos (\omega)$ and $\eta_{y}=y_{m}-\left(\frac{1-(-1)^{m}}{2}\right) L \sin (\omega)$ 



\section{The equivalence of the rigid quantum rotor and semiflexible filament}

We can consider the orientational probability distribution function for free semiflexible filament. Having the tangent vector at initial point of the filament $t(0)=t_{0}$, the probability to find the tangent vector of end point of the filament at value $t(L)=t_{L}$ is given by the following expression:

$$
G\left(t_{L}, s=L \mid t_{0}, s=0\right)=\int_{t(0)=t_{0}}^{t(L)=t_{L}} \exp \left[-\frac{\kappa}{2 k_{B} T} \int_{0}^{L}\left(\frac{\partial \vec{t}}{\partial s}\right)^{2} d s\right] \delta(|t(\vec{s})|-1) D\{t(\vec{s})\}
$$

On the other hand, one can consider a particle with mass $m$ moving on the suface of a sphere with radius of $R$. The system is called rigid rotor. The operator form of the Hamiltonian of the system can be written according to below:

$$
\tilde{H}=-\frac{\hbar^{2}}{2 m} \nabla_{R}^{2}
$$

where $\nabla_{R}^{2}$ is Laplacian living on a sphere with a fixed radius of $R$. The Schrödinger equation of the system with the Hamiltonian is given by the following relation:

$$
\frac{\partial \psi}{\partial t}=\frac{i \hbar}{2 m R^{2}}\left(\frac{1}{\sin (\theta)} \frac{\partial}{\partial \theta} \sin (\theta) \frac{\partial}{\partial \theta}+\frac{1}{\sin ^{2}(\theta)} \frac{\partial^{2}}{\partial \phi^{2}}\right) \psi
$$

Where $\psi$ is the wave function associated with the particle. The wave function is also given by the following path integral:

$$
\psi=\int_{r(0)}^{r(T)} \exp \left[\frac{i}{\hbar} \frac{m}{2} \int_{0}^{T}\left(\frac{\partial \vec{r}}{\partial t}\right)^{2} d t\right] \delta(|r(\vec{t})|-R) D\{r(\vec{t})\}
$$

If we put $R=1$ in the path integral, it become equivalent to the orientational probability distribution function of the semiflexible filament. Therefore one can 
write the following correspondence relations:

$$
\left\{\begin{array}{l}
i \hbar \Leftrightarrow k_{B} T \\
T \Leftrightarrow L \\
m \Leftrightarrow \kappa
\end{array}\right.
$$

This equivalence ensures that the partial differential form of the equivalence is valid too and therefore we can write the following equation for the orientational probability distribution function of the semiflexible filament:

$$
\frac{\partial G}{\partial s}=\frac{k_{B} T}{2 \kappa}\left(\frac{1}{\sin (\theta)} \frac{\partial}{\partial \theta} \sin (\theta) \frac{\partial}{\partial \theta}+\frac{1}{\sin ^{2}(\theta)} \frac{\partial^{2}}{\partial \phi^{2}}\right) G
$$

This is schrödinger-like equation for semiflexible filament. For two dimensional filament, the equation becomes according to below:

$$
\frac{\partial G}{\partial s}=\frac{k_{B} T}{2 \kappa} \frac{\partial^{2} G}{\partial \theta^{2}}
$$

One can replace the $s$ derivative in the left hand side of the equation with convective derivative $\frac{\partial}{\partial s}+t . \nabla$ in order to obtain the partial differential equation for the complete orientational-positional probability distribution function of the semiflexible filament [38]:

$$
\left[\frac{\partial}{\partial s}+\cos (\theta) \frac{\partial}{\partial x}+\sin (\theta) \frac{\partial}{\partial y}-\frac{1}{l_{p}} \frac{\partial^{2}}{\partial \theta^{2}}\right] G_{L}\left(x, y, \theta, s \mid x_{0}, y_{0}, \omega, 0\right)=0
$$




\section{Elasticity of a semiflexible filament with tension discontinuity}

\section{D.1 Filament with hinged-hinged boundary conditions at the two tips}

The force is a piecewise constant function with two pieces. As result of this fact, the Green function is a piecewise function with six pieces:

$$
G\left(s, s^{\prime}\right)= \begin{cases}G_{1}^{-}\left(s, s^{\prime}\right) & 0<s<s^{\prime} \leq L_{m}<L \\ G_{1}^{+}\left(s, s^{\prime}\right) & 0<s^{\prime}<s \leq L_{m}<L \\ G_{2}^{-}\left(s, s^{\prime}\right) & 0<L_{m} \leq s<s^{\prime}<L \\ G_{2}^{+}\left(s, s^{\prime}\right) & 0<L_{m} \leq s^{\prime}<s<L \\ G_{3}^{-}\left(s, s^{\prime}\right) & 0<s<L_{m}<s^{\prime}<L \\ G_{3}^{+}\left(s, s^{\prime}\right) & 0<s^{\prime}<L_{m}<s<L\end{cases}
$$

The assumption that the derivative of the tangent vector is zero at the end tips (hinged-hinged condition) leads to the vanishing of the derivative of the Green function. Considering the boundary conditions, the solution for the aforementioned equation must have the following form:

$$
G_{h h}\left(s, s^{\prime}\right)=\left\{\begin{array}{l}
G_{1}^{-}\left(s, s^{\prime}\right)=N_{11}\left(s^{\prime}\right) \cosh \left(s \sigma_{1}\right) \\
G_{1}^{+}\left(s, s^{\prime}\right)=N_{12}\left(s^{\prime}\right)\left(\cosh \left(s \sigma_{1}\right)+A_{1} \sinh \left(s \sigma_{1}\right)\right) \\
G_{2}^{-}\left(s, s^{\prime}\right)=N_{21}\left(s^{\prime}\right)\left(\cosh \left(s \sigma_{2}\right)+A_{2} \sinh \left(s \sigma_{2}\right)\right) \\
G_{2}^{+}\left(s, s^{\prime}\right)=N_{22}\left(s^{\prime}\right) \cosh \left((L-s) \sigma_{2}\right) \\
G_{3}^{-}\left(s, s^{\prime}\right)=N_{31}\left(s^{\prime}\right) \cosh \left(s \sigma_{1}\right) \\
G_{3}^{+}\left(s, s^{\prime}\right)=N_{32}\left(s^{\prime}\right) \cosh \left((L-s) \sigma_{2}\right)
\end{array}\right.
$$


where $\sigma(s)=\sqrt{\frac{f(s)}{\kappa}}=\left\{\begin{array}{ll}\sigma_{1}=\sqrt{\frac{f_{1}}{\kappa}} & 0<s<L_{m} \\ \sigma_{2}=\sqrt{\frac{f_{2}}{\kappa}} & L_{m}<s<L\end{array}\right.$ and $L_{m}$ is the position of molecular motor in terms of the contour length. Moreover, constants appearing in eq. D.2 are obtained from the following conditions:

$$
\left\{\begin{array}{l}
\left.\frac{\partial G_{1}^{+}\left(s, s^{\prime}\right)}{\partial s}\right|_{s=s^{\prime}}-\left.\frac{\partial G_{1}^{-}\left(s, s^{\prime}\right)}{\partial s}\right|_{s=s^{\prime}}=-\frac{1}{\beta \kappa} \\
G_{1}^{-}\left(s^{\prime}, s^{\prime}\right)=G_{1}^{+}\left(s^{\prime}, s^{\prime}\right) \\
\left.\frac{\partial G_{2}^{+}\left(s, s^{\prime}\right)}{\partial s}\right|_{s=s^{\prime}}-\left.\frac{\partial G_{2}^{-}\left(s, s^{\prime}\right)}{\partial s}\right|_{s=s^{\prime}}=-\frac{1}{\beta \kappa} \\
G_{2}^{-}\left(s^{\prime}, s^{\prime}\right)=G_{2}^{+}\left(s^{\prime}, s^{\prime}\right) \\
G_{3}^{-}\left(L_{m}, s^{\prime}\right)=G_{2}^{-}\left(L_{m}, s^{\prime}\right) \\
G_{3}^{+}\left(L_{m}, s^{\prime}\right)=G_{1}^{+}\left(L_{m}, s^{\prime}\right) \\
\left.\frac{\partial G_{3}^{-}\left(s, s^{\prime}\right)}{\partial s}\right|_{s=L_{m}}=\left.\frac{\partial G_{2}^{-}\left(s, s^{\prime}\right)}{\partial s}\right|_{s=L_{m}} \\
\left.\frac{\partial G_{3}^{+}\left(s, s^{\prime}\right)}{\partial s}\right|_{s=L_{m}}=\left.\frac{\partial G_{1}^{+}\left(s, s^{\prime}\right)}{\partial s}\right|_{s=L_{m}}
\end{array}\right.
$$

These conditions coming in Eq. D.3, except number (7) and number (8), gives:

$$
\left\{\begin{array}{l}
N_{11}\left(s^{\prime}\right)=-\frac{\cosh \left(\sigma_{1} s^{\prime}\right)+A_{1} \sinh \left(\sigma_{1} s^{\prime}\right)}{\sigma_{1} l_{p} A_{1}} \\
N_{12}\left(s^{\prime}\right)=-\frac{\cosh \left(\sigma_{1} s^{\prime}\right)}{\sigma_{1} l_{p} A_{1}} \\
N_{21}\left(s^{\prime}\right)=\frac{\cosh \left(\sigma_{2}\left(L-s^{\prime}\right)\right)}{\sigma_{2} l_{p}\left(\sinh \left(\sigma_{2} L\right)+A_{2} \cosh \left(\sigma_{2} L\right)\right)} \\
N_{22}\left(s^{\prime}\right)=\frac{\cosh \left(\sigma_{2} s^{\prime}\right)+A_{2} \sinh \left(\sigma_{2} s^{\prime}\right)}{\sigma_{2} l_{p}\left(\sinh \left(\sigma_{2} L\right)+A_{2} \cosh \left(\sigma_{2} L\right)\right)} \\
N_{31}\left(s^{\prime}\right)=\frac{\cosh \left(\sigma_{2}\left(L-s^{\prime}\right)\left(\cosh \left(\sigma_{2} L_{m}\right)+A_{2} \sinh \left(\sigma_{2} L_{m}\right)\right)\right)}{\sigma_{2} l_{p} \cosh \left(\sigma_{1} L_{m}\right)\left(\sinh \left(\sigma_{2} L\right)+A_{2} \cosh \left(\sigma_{2} L\right)\right)} \\
N_{32}\left(s^{\prime}\right)=-\frac{\cosh \left(\sigma_{1} s^{\prime}\right)\left(\cosh \left(\sigma_{1} L_{m}\right)+A_{1} \sinh \left(\sigma_{1} L_{m}\right)\right)}{\sigma_{1} l_{p} A_{1} \cosh \left(\sigma_{2}\left(L-L_{m}\right)\right)}
\end{array}\right.
$$

The conditions number (7) and number (8) of eq. D.3 give: 


$$
\left\{\begin{array}{l}
A_{1}=-\frac{\sigma_{2} \sinh \left(\sigma_{2}\left(L-L_{m}\right)\right) \cosh \left(\sigma_{1} L_{m}\right)+\sigma_{1} \sinh \left(\sigma_{1} L_{m}\right) \cosh \left(\sigma_{2}\left(L-L_{m}\right)\right)}{\sigma_{2} \sinh \left(\sigma_{2}\left(L-L_{m}\right)\right) \sinh \left(\sigma_{1} L_{m}\right)+\sigma_{1} \cosh \left(\sigma_{1} L_{m}\right) \cosh \left(\sigma_{2}\left(L-L_{m}\right)\right)} \\
A_{2}=-\frac{\sigma_{1} \sinh \left(\sigma_{1} L_{m}\right) \cosh \left(\sigma_{2} L_{m}\right)-\sigma_{2} \sinh \left(\sigma_{2} L_{m}\right) \cosh \left(\sigma_{1} L_{m}\right)}{\sigma_{1} \sinh \left(\sigma_{1} L_{m}\right) \sinh \left(\sigma_{2} L_{m}\right)-\sigma_{2} \cosh \left(\sigma_{2} L_{m}\right) \cosh \left(\sigma_{1} L_{m}\right)}
\end{array}\right.
$$

Concerning the boundary condition, the correlation function of the transverse components of tangent vector is written as follows:

$$
\left\langle a_{i}(s) a_{i}\left(s^{\prime}\right)\right\rangle_{h h}=\lim _{J_{i} \rightarrow 0} \frac{\delta^{2} \ln \left(Z\left(J_{i}\right)\right)}{\delta J_{i}(s) \delta J_{i}\left(s^{\prime}\right)},
$$

where $Z\left(J_{i}\right)=\int D\left\{a_{i}(s)\right\} \delta\left(\int_{0}^{L} d s a_{i}(s)\right) \exp \left(-\beta H_{W B A}+\int_{0}^{L} d s J_{i}(s) a_{i}(s)\right)$ is the generating functional with source term $J_{i}(s)$ and $\beta=\frac{1}{k_{B} T}$. The correlation function of the transverse components of the tangent vector is obtained by the following expression [55, 56]:

$$
\left\langle a_{i}(s) a_{i}\left(s^{\prime}\right)\right\rangle=G_{h h}\left(s, s^{\prime}\right)-\frac{\int_{o}^{L} G_{h h}\left(s, s_{1}\right) d s_{1} \int_{o}^{L} G_{h h}\left(s^{\prime}, s_{2}\right) d s_{2}}{\int_{0}^{L} \int_{0}^{L} G_{h h}\left(s_{1}, s_{2}\right) d s_{1} d s_{2}}
$$

which implies

$$
\left\langle a_{i}^{2}(s)\right\rangle=G_{h h}(s, s)-\frac{\left(\int_{o}^{L} G_{h h}\left(s, s_{1}\right) d s_{1}\right)^{2}}{\int_{0}^{L} \int_{0}^{L} G_{h h}\left(s_{1}, s_{2}\right) d s_{1} d s_{2}}
$$

\section{D.2 Filament with clamped-free boundary conditions at the two end tips}

In the clamped-free case, we enforce the transverse components of the tangent vector of the filament at $s=0$ and their derivitive at $s=L$ to be zero. Similar to Appendix 
1, we obtain the following expression for the Green function:

$$
G_{c f}\left(s, s^{\prime}\right)=\left\{\begin{array}{l}
G_{1}^{-}\left(s, s^{\prime}\right) \equiv \frac{\sinh \left(\sigma_{1} s\right)\left(B_{1} \cosh \left(\sigma_{1} s^{\prime}\right)+\sinh \left(\sigma_{1} s^{\prime}\right)\right)}{l_{p} \sigma_{1} B_{1}} \\
G_{1}^{+}\left(s, s^{\prime}\right) \equiv \frac{\left(B_{1} \cosh \left(\sigma_{1} s\right)+\sinh \left(\sigma_{1} s\right)\right) \sinh \left(\sigma_{1} s^{\prime}\right)}{l_{p} \sigma_{1} B_{1}} \\
G_{2}^{-}\left(s, s^{\prime}\right) \equiv \frac{\cosh \left(\sigma_{2}\left(L-s^{\prime}\right)\right)\left(B_{2} \cosh \left(\sigma_{2} s\right)+\sinh \left(\sigma_{2} s\right)\right)}{l_{p} \sigma_{2} B_{2} \sinh \left(\sigma_{2} L\right)+l_{p} \sigma_{2} \cosh \left(\sigma_{2} L\right)} \\
G_{2}^{+}\left(s, s^{\prime}\right) \equiv \frac{\cosh \left(\sigma_{2} s\right)\left(B_{2} \cosh \left(\sigma_{2} s^{\prime}\right)+\sinh \left(\sigma_{2} s^{\prime}\right)\right)}{l_{p} \sigma_{2} B_{2} \sinh \left(\sigma_{2} L\right)+l_{p} \sigma_{2} \cosh \left(\sigma_{2} L\right)} \\
G_{3}^{-}\left(s, s^{\prime}\right) \equiv \frac{\sinh \left(\sigma_{1} s\right) \cosh \left(\sigma_{2}\left(L-s^{\prime}\right)\right)\left(B_{2} \cosh \left(\sigma_{2} L_{m}\right)+\sinh \left(\sigma_{2} L_{m}\right)\right)}{l_{p} \sigma_{2} \sinh \left(\sigma_{1} L_{m}\right)\left(B_{2} \sinh \left(\sigma_{2} L\right)+\cosh \left(\sigma_{2} L\right)\right)} \\
G_{3}^{+}\left(s, s^{\prime}\right) \equiv \frac{\cosh \left(\sigma_{2}(L-s)\right) \sinh \left(\sigma_{1} s^{\prime}\right)\left(B_{1} \cosh \left(\sigma_{1} L_{m}\right)+\sinh \left(\sigma_{1} L_{m}\right)\right)}{l_{p} \sigma_{1} B_{1} \cosh \left(\sigma_{2}\left(L-L_{m}\right)\right)}
\end{array}\right.
$$

where:

$$
\left\{\begin{array}{l}
B_{1}=-\frac{\sigma_{2} \sinh \left(\sigma_{2}\left(L-L_{m}\right)\right) \sinh \left(\sigma_{1} L_{m}\right)+\sigma_{1} \cosh \left(\sigma_{2}\left(L-L_{m}\right)\right) \cosh \left(\sigma_{1} L_{m}\right)}{\sigma_{2} \sinh \left(\sigma_{2}\left(L-L_{m}\right)\right) \cosh \left(\sigma_{1} L_{m}\right)+\sigma_{1} \cosh \left(\sigma_{2}\left(L-L_{m}\right)\right) \sinh \left(\sigma_{1} L_{m}\right)} \\
B_{2}=\frac{\sigma_{1} \cosh \left(\sigma_{1} L_{m}\right) \sinh \left(\sigma_{2} L_{m}\right)-\sigma_{2} \sinh \left(\sigma_{1} L_{m}\right) \cosh \left(\sigma_{2} L_{m}\right)}{\sigma_{2} \sinh \left(\sigma_{1} L_{m}\right) \sinh \left(\sigma_{2} L_{m}\right)-\sigma_{1} \cosh \left(\sigma_{1} L_{m}\right) \cosh \left(\sigma_{2} L_{m}\right)}
\end{array}\right.
$$

The correlation function [55, 56] is obtained as follows:

$$
\left\langle a_{i}(s) a_{i}\left(s^{\prime}\right)\right\rangle_{c f}=G_{c f}\left(s, s^{\prime}\right)
$$

which implies

$$
\left\langle a_{i}^{2}(s)\right\rangle_{c f}=G_{c f}(s, s)
$$

\section{D.3 Linear end to end distance in terms of $f_{m}$}

The end to end distance of the whole filament when it is in the clamped-free condition can be written as follows:

$$
\langle R\rangle_{c f}=L-\frac{L^{2} \tanh \left(\tilde{f}_{e x t}^{\frac{1}{2}}\right)}{2 l_{p} \tilde{f}_{e x t}}+\left(\frac{1}{k\left(f_{\text {ext }}\right)}\right) f_{m}
$$


The effective linear motor force constant $\mathrm{k}$ is:

$$
\begin{aligned}
\frac{1}{k}= & -\frac{1}{4\left(l_{p} \kappa\left(\frac{f_{e x t}}{\kappa}\right)^{\frac{5}{2}}\left(e^{4 L \sqrt{\frac{f_{e x t}}{\kappa}}}+2 e^{2 L \sqrt{\frac{f_{e x t}}{\kappa}}}+1\right)\right)} \\
& \times\left[A e^{4 L \sqrt{\frac{f_{e x t}}{\kappa}}}+B e^{2\left(2 L-L_{m}\right) \sqrt{\frac{f_{e x t}}{\kappa}}}+C e^{2 L \sqrt{\frac{f_{e x t}}{\kappa}}}+D e^{2\left(L+L_{m}\right) \sqrt{\frac{f_{e x t}}{\kappa}}}+E e^{2\left(L-L_{m}\right) \sqrt{\frac{f_{e x t}}{\kappa}}}\right. \\
& \left.+F e^{2 L_{m} \sqrt{\frac{f_{e x t}}{\kappa}}}+G\right],
\end{aligned}
$$

where $A=-\frac{f_{e x t} L_{m}}{\kappa}+\sqrt{\frac{f_{e x t}}{\kappa}}, B=-\left(\frac{f_{e x t} L_{m}}{\kappa}+\sqrt{\frac{f_{e x t}}{\kappa}}\right), C=\left(\frac{4 f_{e x t} L L_{m}}{\kappa}+2\right) \sqrt{\frac{f_{e x t}}{\kappa}}, D=$ $-\left(\sqrt{\frac{f_{e x t}}{\kappa}}-\frac{f_{e x t} L_{m}}{\kappa}+\frac{f_{e x t} L}{\kappa}\right), E=-\sqrt{\frac{f_{e x t}}{\kappa}}-\frac{f_{e x t} L_{m}}{\kappa}+\frac{f_{e x t} L}{\kappa}, F=\frac{f_{e x t} L_{m}}{\kappa}-\sqrt{\frac{f_{e x t}}{\kappa}}, G=$ $\frac{f_{e x t} L_{m}}{\kappa}+\sqrt{\frac{f_{\text {ext }}}{\kappa}}$

\section{D.4 Limit of large motor force $f_{m}$}

In the limit of large motor forces $f_{m} \gg \operatorname{Max}\left\{f_{\text {ext }}, \frac{\kappa}{L^{2}}\right\}$, we have the following expression for the end to end distance of the first piece of the filament:

$$
\left\langle R_{1}\right\rangle_{c f}=L_{m}-\frac{L_{m}}{2 l_{p}} \sqrt{\frac{\kappa}{f_{m}}}
$$

and the end to end distance of the second piece of the filament:

$$
\begin{aligned}
\left\langle R_{2}\right\rangle_{c f}=\left(L-L_{m}\right) & -\frac{\left(L-L_{m}\right)}{2 l_{p}} \frac{\tanh \left(\left(L-L_{m}\right) \sqrt{\frac{f_{e x t}}{\kappa}}\right)}{\sqrt{\frac{f_{e x t}}{\kappa}}} \\
& -\alpha\left(f_{\text {ext }}\right) \sqrt{\frac{\kappa}{f_{m}}}
\end{aligned}
$$

where

$$
\begin{aligned}
\alpha\left(f_{\text {ext }}\right)= & \frac{e^{4 L \sqrt{\frac{f_{e x t}}{\kappa}}}-e^{4 L_{m} \sqrt{\frac{f_{e x t}}{\kappa}}}}{2 l_{p} \sqrt{\frac{f_{e x t}}{\kappa}}\left(e^{2 L \sqrt{\frac{f_{e x t}}{\kappa}}}+e^{\left.2 L_{m} \sqrt{\frac{f_{e x t}}{\kappa}}\right)^{2}}\right.} \\
+ & \frac{2\left(L-L_{m}\right) e^{2\left(L+L_{m}\right) \sqrt{\frac{f_{e x t}}{\kappa}}}}{l_{p}\left(e^{2 L \sqrt{\frac{f_{e x t}}{\kappa}}}+e^{2 L_{m} \sqrt{\frac{f_{e x t}}{\kappa}}}\right)^{2}}
\end{aligned}
$$





\section{E The corelation Function of components of the tangent vector}

\section{E.1 clamped-free case}

The generating functional with source term $J_{i}(s)$ is written according to below

$$
Z\left(J_{i}\right)=\int D\left\{a_{i}(s)\right\} \exp \left(-\beta H_{W B}+\int_{0}^{L} d s J_{i}(s) a_{i}(s)\right)
$$

Where $\beta=\frac{1}{k_{B} T}$ and $i=1,2$ and we have Einstein summation rule. The correlation function of the transverse components of the tangent vector is obtained by the following expression:

$$
\left\langle a_{i}(s) a_{i}\left(s^{\prime}\right)\right\rangle_{c f}=\lim _{J_{i} \rightarrow 0} \frac{\delta^{2} \ln \left(Z\left(J_{i}\right)\right)}{\delta J_{i}(s) \delta J_{i}\left(s^{\prime}\right)}
$$

According to [56] the above mentioned correlation function reads:

$$
\left\langle a_{i}(s) a_{i}\left(s^{\prime}\right)\right\rangle_{c f}=G_{c f}\left(s, s^{\prime}\right)
$$

Where $G_{c f}\left(s, s^{\prime}\right)$ is the green's function of operator $\beta O(s)$ and the symbol $c f$ refers to the clamped-free condition for the filament. The Green's function should satisfy the following relations:

$$
\left\{\begin{array}{l}
\beta\left(-\kappa \frac{d^{2}}{d s^{2}}+f(s)\right) G_{c f}\left(s, s^{\prime}\right)=\delta\left(s-s^{\prime}\right) \\
\left.G_{c f}\left(s, s^{\prime}\right)\right|_{s=0}=0 \\
\left.\frac{\partial}{\partial s} G_{c f}\left(s, s^{\prime}\right)\right|_{s=L}=0
\end{array}\right.
$$

\section{E.2 Hinged-hinged case}

The generating functional with source term $J_{i}(s)$ is written according to below

$$
Z\left(J_{i}\right)=\int D\left\{a_{i}(s)\right\} \delta\left(\int_{0}^{L} d s a_{i}(s)\right) \exp \left(-\beta H_{W B}+\int_{0}^{L} d s J_{i}(s) a_{i}(s)\right)
$$


Where $\beta=\frac{1}{k_{B} T}$ and $i=1,2$. In this appendix, we want to prove that simplified expression for correlation function the transverse components is:

$$
\begin{aligned}
\left\langle a_{i}(s) a_{i}\left(s^{\prime}\right)\right\rangle_{h h} & =-\frac{\int_{o}^{L} G_{h h}\left(s, s_{1}\right) d s_{1} \int_{o}^{L} G_{h h}\left(s^{\prime}, s_{2}\right) d s_{2}}{\int_{0}^{L} \int_{0}^{L} G_{h h}\left(s_{1}, s_{2}\right) d s_{1} d s_{2}} \\
& +G_{h h}\left(s, s^{\prime}\right)
\end{aligned}
$$

Where $G_{h h}\left(s, s^{\prime}\right)$ is the Green's function of the filament with hinged-hinged condition. The Green's function should satisfy the following relations:

$$
\left\{\begin{array}{l}
\beta\left(-\kappa \frac{d^{2}}{d s^{2}}+f(s)\right) G_{h h}\left(s, s^{\prime}\right)=\delta\left(s-s^{\prime}\right) \\
\left.\frac{\partial}{\partial s} G_{h h}\left(s, s^{\prime}\right)\right|_{s=0}=0 \\
\left.\frac{\partial}{\partial s} G_{h h}\left(s, s^{\prime}\right)\right|_{s=L}=0
\end{array}\right.
$$

Using Fourier representation of Dirac function, We can rewrite the generating functional as below:

$$
Z\left(J_{n}\right)=\int D\left\{a_{n}(s)\right\} \int \frac{d \vec{k}}{4 \pi^{2}} \exp \left(-\beta H_{W B}+\int_{0}^{L} d s J_{n}(s) a_{n}(s)+i k_{n} \int_{0}^{L} d s a_{n}(s)\right)
$$

Where $\vec{k}=\left(k_{1}, k_{2}\right)$. Defining $\tilde{J}_{n}(s)=J_{n}(s)+i k_{n}$, we have:

$$
Z\left(J_{i}\right)=\int D\left\{a_{i}(s)\right\} \int \frac{d \vec{k}}{4 \pi^{2}} \exp \left(-\beta H_{W B}+\int_{0}^{L} d s \tilde{J}_{i}(s) a_{i}(s)\right)
$$

Now, the above mentioned generating functional is very similar to generating functional of Klein-Gordon field. Completing the square, one can write the generating functional according to below:

$$
Z\left(J_{i}\right)=Z_{0} \int \frac{d \vec{k}}{4 \pi^{2}} \exp \left(-\frac{1}{2} \int_{0}^{L} d s_{1} d s_{2} \tilde{J}_{i}^{*}\left(s_{1}\right) G\left(s_{1}, s_{2}\right) \tilde{J}_{i}\left(s_{2}\right)\right)
$$

Where $Z_{0}=\int D\left\{a_{i}(s)\right\} \exp \left(-\beta H_{W B}\right)$. The Green's function is symmetric namely $G\left(s_{1}, s_{2}\right)=G\left(s_{2}, s_{1}\right)$. Using this fact, we rewrite the generating functional:

$$
\begin{aligned}
& Z\left(J_{i}\right)=Z_{0} \int \frac{d \vec{k}}{4 \pi^{2}} \exp \left(-\frac{1}{2} \int_{0}^{L} d s_{1} d s_{2} J_{i}\left(s_{1}\right) G\left(s_{1}, s_{2}\right) J_{i}\left(s_{2}\right)\right) \\
& \times \exp \left(-i k \int_{0}^{L} d s_{1} d s_{2} J_{i}\left(s_{1}\right) G\left(s_{1}, s_{2}\right)-\frac{1}{2} k^{2} \int_{0}^{L} d s_{1} d s_{2} G\left(s_{1}, s_{2}\right)\right) \\
& \Rightarrow
\end{aligned}
$$




$$
\begin{aligned}
Z\left(J_{i}\right) \propto Z_{0} \exp \left(-\frac{1}{2} \int_{0}^{L} d s_{1} d s_{2} J_{i}\left(s_{1}\right) G\left(s_{1}, s_{2}\right) J_{i}\left(s_{2}\right)\right) \\
\quad \times \exp \left(\frac{\left(\int_{0}^{L} d s_{1} d s_{2} J_{i}\left(s_{1}\right) G\left(s_{1}, s_{2}\right)\right)^{2}}{2 \int_{0}^{L} d s_{1} d s_{2} G\left(s_{1}, s_{2}\right)}\right)
\end{aligned}
$$

The correlation function of the transverse components of the tangent vector reads:

$$
\begin{aligned}
<a_{i}(s) a_{i}\left(s^{\prime}\right)>\quad=G\left(s, s^{\prime}\right) & \\
-\frac{\delta}{\delta J(s)} & {\left[\frac{2 \int d s_{2} G\left(s^{\prime}, s_{2}\right) \int d s_{1} d s_{2} J\left(s_{1}\right) G\left(s_{1}, s_{2}\right)}{2 \int d s_{1} d s_{2} G\left(s_{1}, s_{2}\right)}\right] } \\
\Rightarrow & =G\left(s, s^{\prime}\right) \\
<a_{i}(s) a_{i}\left(s^{\prime}\right)>\quad & {\left[\frac{\int d s_{1} G\left(s^{\prime}, s_{1}\right) \int d s_{2} G\left(s, s_{2}\right)}{\int d s_{1} d s_{2} G\left(s_{1}, s_{2}\right)}\right] }
\end{aligned}
$$





\section{Bibliography}

[1] D. Bray. Cell Movements. Garland, New York, 2nd edition, 2001.

[2] Hidekiand Yamaguchi, Jeffrey Wyckoff, and John Condeelis. Cell migration in tumors. Curr Opin Cell Biol, 17:559-564, Oct 2005.

[3] John Condeelis and Jeffrey W. Pollard. Macrophages: Obligate partners for tumor cell migration, invasion, and metastasis. Cell, 124(2):263 - 266, 2006.

[4] J. Victor Small, Theresia Stradal, Emmanuel Vignal, and Klemens Rottner. The lamellipodium: where motility begins. Trends Cell Biol, 12(3):112 - 120, 2002 .

[5] T. M. Svitkina, A. B. Verkhovsky, K. M. McQuade, and G. G. Borisy. Analysis of the Actin-Myosin II System in Fish Epidermal Keratocytes: Mechanism of Cell Body Translocation. J Cell Biol, 139:397 - 415, 1997.

[6] TD Pollard. The cytoskeleton, cellular motility and the reductionist agenda. Nature, 422:741-745, 2003.

[7] Jingyuan Xu, Denis Wirtz, and Thomas D. Pollard. Dynamic cross-linking by $\alpha$-actinin determines the mechanical properties of actin filament networks. $J$ Biol Chem, 273(16):9570-9576, 1998.

[8] Alex Mogilner and George Oster. Force Generation by Actin Polymerization II: The Elastic Ratchet and Tethered Filaments. Biophys J, 84(3):1591-1605, 2003.

[9] M. L. Gardel, J. H. Shin, F. C. MacKintosh, L. Mahadevan, P. Matsudaira, and D. A. Weitz. Elastic behavior of cross-linked and bundled actin networks. Science, 304(5675):1301-1305, 2004.

[10] Sophie Bohnet, Revathi Ananthakrishnan, Alex Mogilner, Jean-Jacques Meister, and Alexander B. Verkhovsky. Weak force stalls protrusion at the leading edge of the lamellipodium. Biophys J, 90(5):1810 - 1820, 2006.

[11] Marcus Prass, Ken Jacobson, Alex Mogilner, and Manfred Radmacher. Direct measurement of the lamellipodial protrusive force in a migrating cell. J Cell Biol, 174(6):767-772, 2006. 
[12] Fabian Heinemann, Holger Doschke, and Manfred Radmacher. Keratocyte lamellipodial protrusion is characterized by a concave force-velocity relation. Biophys J, 100(6):1420 - 1427, 2011.

[13] Juliane Zimmermann, Claudia Brunner, Mihaela Enculescu, Michael Goegler, Allen Ehrlicher, Josef Käs, and Martin Falcke. Actin filament elasticity and retrograde flow shape the force-velocity relation of motile cells. Biophys $J$, 102(2):287 - 295, 2012.

[14] Fumihiko Nakamura, Eric Osborn, Paul A. Janmey, and Thomas P. Stossel. Comparison of filamin a-induced cross-linking and arp2/3 complex-mediated branching on the mechanics of actin filaments. J Biol Chem, 277(11):91489154, 2002.

[15] Tatyana M. Svitkina and Gary G. Borisy. Arp2/3 complex and actin depolymerizing factor/cofilin in dendritic organization and treadmilling of actin filament array in lamellipodia. J Cell Biol, 145(5):1009-1026, 1999.

[16] A.B. Verkhovsky, T.M. Svitkina, and G.G. Borisy. Self-polarization and directional motility of cytoplasm. Curr Biol, 9(1):11-20, 1999.

[17] Pascal Vallotton, Gaudenz Danuser, Sophie Bohnet, Jean-Jacques Meister, and Alexander B. Verkhovsky. Tracking Retrograde Flow in Keratocytes: News from the Front. Mol Biol Cell, 16(3):1223-1231, 2005.

[18] Kinneret Keren, Zachary Pincus, Greg M. Allen, Erin L. Barnhart, Gerard Marriott, Alex Mogilner, and Julie A. Theriot. Mechanism of shape determination in motile cells. Nature, 453(7194):475-480, 2008.

[19] Juliane Zimmermann, Mihaela Enculescu, and Martin Falcke. Leading edge gel coupling in lamellipodium motion. Phys Rev E, 82(5):051925, Nov 2010.

[20] Boris Rubinstein, Maxime F. Fournier, Ken Jacobson, Alexander B. Verkhovsky, and Alex Mogilner. Actin-myosin viscoelastic flow in the keratocyte lamellipod. Biophys J, 97(7):1853 - 1863, 2009.

[21] Mihaela Enculescu, Mohsen Sabouri-Ghomi, Gaudenz Danuser, and Martin Falcke. Modeling of protrusion phenotypes driven by the actin-membrane interaction. Biophys J, 98(8):1571 - 1581, 2010.

[22] Erin L. Barnhart, Kun-Chun Lee, Kinneret Keren, Alex Mogilner, and Julie A. Theriot. An adhesion-dependent switch between mechanisms that determine motile cell shape. PLoS Biol, 9(5):e1001059, 052011. 
[23] Danying Shao, Herbert Levine, and Wouter-Jan Rappel. Coupling actin flow, adhesion, and morphology in a computational cell motility model. Proc Nat Acad Sci USA, 109(18):6851-6856, 2012.

[24] M. Falcke and J. Zimmermann. Polymerization, bending, tension: What happens at the leading edge of motile cells? EPJ ST, 223(7):1353-1372, 2014.

[25] Robyn H. Pritchard, Yan Yan Shery Huang, and Eugene M. Terentjev. Mechanics of biological networks: from the cell cytoskeleton to connective tissue. Soft Matter, 10:1864-1884, 2014.

[26] C. P. Broedersz and F. C. MacKintosh. Modeling semiflexible polymer networks. Rev. Mod. Phys., 86:995-1036, Jul 2014.

[27] Oliver Lieleg, Mireille M. A. E. Claessens, and Andreas R. Bausch. Structure and dynamics of cross-linked actin networks. Soft Matter, 6:218-225, 2010.

[28] Steven J. Winder and Kathryn R. Ayscough. Actin-binding proteins. Journal of Cell Science, 118(4):651-654, 2005.

[29] Eckhard Mandelkow and Eva-Maria Mandelkow. Microtubules and microtubule-associated proteins. Current Opinion in Cell Biology, 7(1):72 81, 1995.

[30] Claudia Veigel and Christoph F. Schmidt. Moving into the cell: single-molecule studies of molecular motors in complex environments. Nat Rev Mol Cell Biol, 12(3):163-176, 032011.

[31] Erika LF Holzbaur and Yale E Goldman. Coordination of molecular motors: From in vitro assays to intracellular dynamics. Current opinion in cell biology, 22(1):4-13, 022010.

[32] A. F. Huxley. Muscle structure and theories of contraction. PROGRESS IN BIOPHYSICS AND BIOPHYSICAL CHEMISTRY, 7:255-318, 1957.

[33] Thomas Guérin, Jacques Prost, Pascal Martin, and Jean-François Joanny. Coordination and collective properties of molecular motors: theory. Current Opinion in Cell Biology, 22(1):14-20, 22010.

[34] Mohammadhosein Razbin, Martin Falcke, Panayotis Benetatos, and Annette Zippelius. Mechanical properties of branched actin filaments. Physical biology, 12(4):046007, 2015.

[35] Mohammadhosein Razbin, Panayotis Benetatos, and Annette Zippelius. Elasticity of a semiflexible filament with a discontinuous tension due to a cross-link or a molecular motor. Phys. Rev. E, 93:052408, May 2016. 
[36] Azam Gholami, Jan Wilhelm, and Erwin Frey. Entropic forces generated by grafted semiflexible polymers. Phys Rev E, 74(4):041803, Oct 2006.

[37] Johan RC Van der Maarel. Introduction to biopolymer physics. World Scientific, 2008.

[38] P. Benetatos and E. Frey. Depinning of semiflexible polymers. Phys. Rev. E, 67:051108, May 2003.

[39] Stefan A. Koestler, Sonja Auinger, Marlene Vinzenz, Klemens Rottner, and J. Victor Small. Differentially oriented populations of actin filaments generated in lamellipodia collaborate in pushing and pausing at the cell front. Nat Cell Biol, 10(3):306-313, 2008.

[40] Ivan V. Maly and Gary G. Borisy. Self-organization of a propulsive actin network as an evolutionary process. Proceedings of the National Academy of Sciences, 98(20):11324-11329, 2001.

[41] W Urban, S Jacob, M Nemethova, GP Resch, and JV Small. Electron tomography reveals unbranched networks of actin filaments in lamellipodia. Nat Cell Biol, 12:429-435, 2010.

[42] J.V. Small, C. Winkler, M. Vinzenz, and C. Schmeiser. Reply: Visualizing branched actin filaments in lamellipodia by electron tomography. Nat Cell Biol, 13(9):1013-1014, 2011.

[43] Alexander B. Verkhovsky, Oleg Y. Chaga, Sebastien Schaub, Tatyana M. Svitkina, Jean-Jacques Meister, and Gary G. Borisy. Orientational Order of the Lamellipodial Actin Network as Demonstrated in Living Motile Cells. Mol Biol Cell, 14(11):4667-4675, 2003.

[44] Julian Weichsel, Edit Urban, J. Victor Small, and Ulrich S. Schwarz. Reconstructing the orientation distribution of actin filaments in the lamellipodium of migrating keratocytes from electron microscopy tomography data. Cytometry Part A, 81A(6):496-507, 2012.

[45] Christian H. Schreiber, Murray Stewart, and Thomas Duke. Simulation of cell motility that reproduces the force-velocity relationship. Proc Nat Acad Sci USA, 107(20):9141-9146, 2010.

[46] Dylan T. Burnette, Suliana Manley, Prabuddha Sengupta, Rachid Sougrat, Michael W. Davidson, Bechara Kachar, and Jennifer Lippincott-Schwartz. A role for actin arcs in the leading-edge advance of migrating cells. Nat Cell Biol, 13(4):371-382, 2011. 
[47] Marlene Vinzenz, Maria Nemethova, Florian Schur, Jan Mueller, Akihiro Narita, Edit Urban, Christoph Winkler, Christian Schmeiser, Stefan A. Koestler, Klemens Rottner, Guenter P. Resch, Yuichiro Maeda, and J. Victor Small. Actin branching in the initiation and maintenance of lamellipodia. J Cell Sci, 125(11):2775-2785, 2012.

[48] Sabine Petry, AaronÂăC. Groen, Keisuke Ishihara, TimothyÂăJ. Mitchison, and RonaldÂăD. Vale. Branching microtubule nucleation in xenopus egg extracts mediated by augmin and \{TPX2\}. Cell, 152(4):768 - 777, 2013.

[49] F Gittes, B Mickey, J Nettleton, and J Howard. Flexural rigidity of microtubules and actin filaments measured from thermal fluctuations in shape. The Journal of Cell Biology, 120(4):923-934, 1993.

[50] Helmut Schiessel. The physics of chromatin. Journal of Physics: Condensed Matter, 15(19):R699, 2003.

[51] Nam-Kyung Lee, Jin-Sung Park, Albert Johner, Sergei Obukhov, Ju-Yong Hyon, Kyoung J. Lee, and Seok-Cheol Hong. Elasticity of cisplatin-bound dna reveals the degree of cisplatin binding. Phys. Rev. Lett., 101:248101, Dec 2008.

[52] Joseph V Kurian. A new polymer platform for the futureâĂ $̌$ sorona $囚$ from corn derived 1, 3-propanediol. Journal of Polymers and the Environment, 13(2):159$167,2005$.

[53] John F. Marko and Eric D. Siggia. Stretching dna. Macromolecules, 28(26):8759-8770, 1995.

[54] Azam Gholami, Jan Wilhelm, and Erwin Frey. Entropic forces generated by grafted semiflexible polymers. Phys. Rev. E, 74:041803, Oct 2006.

[55] Yuko Hori, Ashok Prasad, and Jané Kondev. Stretching short biopolymers by fields and forces. Physical Review E, 75(4):041904, 2007.

[56] Michael E Peskin and Daniel V Schroeder. An introduction to quantum field theory. Westview, 1995.

[57] Jeffrey T. Finer, Robert M. Simmons, and James A. Spudich. Single myosin molecule mechanics: piconewton forces and nanometre steps. Nature, 368(6467):113-119, 031994.

[58] Claudia Veigel, Fei Wang, Marc L. Bartoo, James R. Sellers, and Justin E. Molloy. The gated gait of the processive molecular motor, myosin v. Nat Cell Biol, 4(1):59-65, 012002. 
[59] Claudia Veigel, Marc L. Bartoo, David C. S. White, John C. Sparrow, and Justin E. Molloy. The stiffness of rabbit skeletal actomyosin cross-bridges determined with an optical tweezers transducer. Biophysical Journal, 75:1424, 1998.

[60] Ulrich Gerland, Ralf Bundschuh, and Terence Hwa. Mechanically probing the folding pathway of single rna molecules. Biophysical Journal, 84(5):2831-2840, 052003.

[61] Panayotis Benetatos, Stephan Ulrich, and Annette Zippelius. ForceâĂŞextension relation of cross-linked anisotropic polymer networks. New Journal of Physics, 14(11):115011, 2012.

[62] Alice von der Heydt, Daniel Wilkin, Panayotis Benetatos, and Annette Zippelius. Elasticity of cross-linked semiflexible biopolymers under tension. Phys. Rev. E, 88:032701, Sep 2013.

[63] Panayotis Benetatos, Alice von der Heydt, and Annette Zippelius. Tensioninduced binding of semiflexible biopolymers. New Journal of Physics, 16(11):113037, 2014.

[64] T. B. Liverpool, M. C. Marchetti, J.-F. Joanny, and J. Prost. Mechanical response of active gels. EPL (Europhysics Letters), 85(1):18007, 2009.

[65] R. Dyche Mullins, John A. Heuser, and Thomas D. Pollard. The interaction of Arp2/3 complex with actin: Nucleation, high affinity pointed end capping, and formation of branching networks of filaments. Proc Nat Acad Sci USA, 95(11):6181-6186, 1998.

[66] Niels Volkmann, Kurt J. Amann, Svetla Stoilova-McPhie, Coumaran Egile, Dirk C. Winter, Larnele Hazelwood, John E. Heuser, Rong Li, Thomas D. Pollard, and Dorit Hanein. Structure of arp2/3 complex in its activated state and in actin filament branch junctions. Science, 293(5539):2456-2459, 2001.

[67] Yang C. and Svitkina T. Visualizing branched actin filaments in lamellipodia by electron tomography. Nat Cell Biol, 13(9):1012-1013, 2011.

[68] James E. Bear, Tatyana M. Svitkina, Matthias Krause, Dorothy A. Schafer, Joseph J. Loureiro, Geraldine A. Strasser, Ivan V. Maly, Oleg Y. Chaga, John A. Cooper, Gary G. Borisy, and Frank B. Gertler. Antagonism between ena/vasp proteins and actin filament capping regulates fibroblast motility. Cell, 109(4):509 - 521, 2002.

[69] Loïc Le Goff, Oskar Hallatschek, Erwin Frey, and François Amblard. Tracer studies on f-actin fluctuations. Phys Rev Lett, 89(25):258101, Dec 2002. 
[70] H Isambert, P Venier, AC Maggs, A Fattoum, R Kassab, D Pantaloni, and MF Carlier. Flexibility of actin filaments derived from thermal fluctuations. effect of bound nucleotide, phalloidin, and muscle regulatory proteins. J Biol Chem, 270(19):11437-11444, 1995.

[71] Brannon R. McCullough, Laurent Blanchoin, Jean-Louis Martiel, and Enrique M. De La Cruz. Cofilin increases the bending flexibility of actin filaments: Implications for severing and cell mechanics. J Mol Biol, 381(3):550 - 558, 2008 .

[72] Jim Pfaendtner, Enrique M. De La Cruz, and Gregory A. Voth. Actin filament remodeling by actin depolymerization factor/cofilin. Proc Nat Acad Sci USA, 107(16):7299-7304, 2010. 



\section{Acknowledgement}

During my stay in Göttingen, a lot of people have helped me. First of all, I would like to thank Annette Zippelius for her kind help and supervision. In spite of fact that my M.Sc. was in different topic (General Relativity), she trusted me and accepted me as her Ph.D. student and she has supervised me with patience and kindness during my study.

I sincerely thank Reiner Kree for accepting to be my second advisor. I thank Marcus Müller, Sarah Köster, Stefan Klumpp and Salvatore R. Manmana for being my examiners.

I thank Panayotis Benetatos and Martin Falcke and Richard L.C. Vink for the fruitful collaborations. During these colaborations, I earned a lot of valuable scientific experience and beside that, friendship with them will remain in my memory as part of the happy times during my study.

I would like to thank my family and friends for their kind support and help.

Also, I sincerely acknowledge the financial support from DFG through Grant No.

SFB 937, Project A1 and the financial support from Max Planck Institute for Dynamics and Self-Organization. 



\section{CV: \\ Mohammadhosein Razbin Khalilabad}

\section{Born:}

16.09.1987 In Ardebil, Iran

Nationality:

Iran

Education

M.Sc, Physics

Sharif University of Technology, Tehran, IRAN, 2009-2011.

B.Sc, Physics

Sharif University of Technology, Tehran, IRAN, 2005-2009.

\section{Research Interests}

Statistical Physics and Biophysics.

\section{Journal Publication}

1) Elasticity of a semiflexible filament with a discontinuous tension due to a crosslink or a molecular motor

Mohammadhosein Razbin, Panayotis Benetatos , Annette Zippelius. Physical Review E (2016)

2) Mechanical properties of branched actin filaments

Mohammadhosein Razbin, Martin Falcke, Panayotis Benetatos, Annette Zippelius. Physical Biology,(2015)

3) Relativistic rotation curve for cosmological structures

Mohammadhosein Razbin, Javad T. Firouzjaee, and Reza Mansouri. International Journal of Modern Physics D 23.09 (2014)

\section{$\underline{\text { Teaching experience }}$}


1) Teaching Assistant, University of Göttingen

Classical mechanics, winter semester, 2013-2014

2) Teaching Assistant, University of Göttingen

Classical field theory,summer semester, 2013

3) Teaching Assistant, Sharif University Of Technology Special Relativity, Spring 2010

4) Teaching Assistant, Sharif University Of Technology Cosmology, Fall 2010

\section{Computer skills}

Programming Languages: $\mathrm{C}++$ Math Packages: Maple 